UNIVERSIDADE DA BEIRA INTERIOR

Engenharia

\title{
Evaluation of Numerical Variable Density Approach to Cryogenic Jets
}

\author{
Eduardo Luís Santos Farias Antunes \\ Dissertação para obtenção do Grau de Mestre em \\ Engenharia Aeronáutica \\ ( $2^{\circ}$ ciclo de estudos)
}

Orientador: Professor Doutor Jorge Manuel Martins Barata

Covilhã, Junho de 2011 
Eduardo Luís Santos Farias Antunes 


\section{Agradecimentos}

Quero agradecer ao meu orientador, Professor Doutor Jorge Manuel Martins Barata, pelas excelentes aulas de Propulsão 1 onde aprendi muito e ganhei ainda maior paixão pela área, pela oportunidade que me deu de trabalhar com ele e pelo enorme conhecimento, ajuda e encorajamento que me prestou.

Quero agradecer ao Professor Doutor André Resende Rodrigues da Silva, pela ajuda e apoio que me prestou durante a execução desta tese principalmente na documentação e apoio bibliográfico.

Quero agradecer ao Engenheiro Fernando Manuel Silva Pereira Neves pela disponibilidade, apoio e encorajamento que me prestou principalmente durante a minha fase de aprendizagem.

Quero agradecer a todos os meus amigos e colegas, pelo apoio moral, encorajamento e conhecimento que me prestaram durante todo o meu percurso académico.

Por último, mas não menos importante, quero agradecer a toda a minha família, em especial, aos meus pais e irmã por sempre me terem apoiado e acreditado em mim incondicionalmente.

Eduardo Antunes

Covilhã 2011 
Eduardo Luís Santos Farias Antunes 


\section{Resumo}

O presente trabalho dedica-se ao estudo de jactos de azoto líquido criogênico em condições subcríticas perto do ponto crítico. A injecção de combustível é um dos maiores desafios actuais na engenharia de motores diesel, turbinas de gás e motores de foguete, com a complexidade extra de nestes últimos se combinar também a injecção de comburente. É amplamente conhecido que o aumento das pressões e temperaturas de operação aumenta a eficiências dos motores e reduz o consumo específico. Assim, existe a tendência de um aumento generalizado das pressões de operação em motores modernos. No entanto elevadas pressões de operação na câmara de combustão têm como consequência a exposição dos fluidos a condições que excedem os valores críticos. Vários autores concluíram que nestas condições os fluidos injectados sofrem variações nas suas propriedades que levam a que os modelos tradicionais de escoamentos com duas fases não consigam modelar correctamente o comportamento do jacto, assim são precisos novos modelos computacionais para estas condições específicas. Barata et al. [18] realizaram uma investigação numérica com o intuito de avaliar a aplicabilidade do modelo de gases de densidade variável em jactos líquidos em condições sub e supercríticas. Os resultados de Barata et al. [18] revelaram uma boa concordância com os dados experimentais, mas apenas foram consideradas razões de densidade intermédias de 0.05 a 0.14 . 0 objectivo do presente trabalho é o de estender as investigações de Ref. 18 a razões de densidades mais baixas entre 0.025 e 0.045 que correspondem a casos de pressões subcríticas da câmara de injecção e determinar o limite de aplicabilidade do modelo de gases de densidade variável. Os resultados obtidos estão em concordância com os dados experimentais e numéricos obtidos por Chehroudi et al. apresentados em Ref. 18. Neste trabalho verificou-se também que o modelo computacional usado não oferece resultados validos para razões de densidade abaixo de 0.025 identificandose assim o limite de aplicabilidade do modelo de gases de densidade variável.

\section{Palavras-chave}

Injecção de combustível; ponto crítico; condições supercríticas; motores foguete; motores diesel; razão de densidades; jactos; densidade variável. 
Eduardo Luís Santos Farias Antunes 


\section{Resumo alargado}

A injecção de combustível apresenta-se como um dos maiores desafios actuais da engenharia em motores diesel, turbinas de gás e foguetes, sendo que neste ultimo caso se acrescenta ainda a complexidade extra da injecção combinada de oxidante. Estudos experimentais e numéricos efectuados para vários tipos de motores têm vindo a demonstrar que o aumento da pressão de funcionamento dos mesmos aumenta bastante a sua eficiência e reduz o consumo específico. Assim antevê-se que seja de todo o interesse a continuação do aumento das pressões de operação dos motores, o que, com o aparecimento de materiais inovadores mais resistentes, faz crescer ainda mais esta tendência.

No entanto, o aumento da pressão de funcionamento leva a que se atinjam e se ultrapassem os pontos críticos de pressão e temperatura dos líquidos e gases envolvidos. Como exemplos, a câmara de combustão do motor principal do Space Shuttle atinge uma pressão de $22.3 \mathrm{MPa}$ enquanto no motor Vulcain, usado no Ariane 5 , se registou um valor recorde de $28.2 \mathrm{MPa}$ [1]. Em qualquer dos casos as pressões na câmara de combustão ultrapassam a pressão critica de $P_{c r}=5.043 \mathrm{MPa}$ para o oxigénio liquido e $\mathrm{P}_{\mathrm{cr}}=1.28 \mathrm{MPa}$ do hidrogénio liquido. Também em motores diesel a câmara combustão pode em alguns casos atingir o dobro da pressão crítica. Para aplicações em motores foguete a temperatura inicial do oxigénio injectado está abaixo do valor crítico $\left(T_{c r}=154.58 \mathrm{~K}\right)$, mas assim que este entra na câmara de combustão passa por uma transição na qual atinge temperaturas supercríticas. Nestas condições diz-se que o combustível (ou comburente líquido) está em condições supercríticas e o seu estado físico passa a ser denominado de estado fluido [1]. Á medida que o fluido alcança condições de pressão e temperatura que ultrapassam os valores críticos, o fluido passa por alterações significativas nas suas propriedades. A difusibilidade mássica efectiva, a tensão superficial e o calor latente do líquido desaparecem em condições supercríticas. Por outro lado, o calor específico a pressão constante, $C_{p}$, a compressibilidade isentrópica, $\kappa_{s}$, e a condutividade térmica, $\lambda$, tornam-se infinitos [2]. Estas mudanças de comportamento do fluido fazem com que os tradicionais modelos de escoamentos com duas fases não possam ser aplicados em injecções de combustível em condições supercríticas. Desta forma, nasce a necessidade de desenvolver novos modelos computacionais que possam ser correctamente aplicado à injecção de combustível em condições supercríticas.

Vários autores investigaram a injecção de combustível em condições supercríticas tanto experimentalmente como numericamente [3-22]. As primeiras investigações experimentais realizadas tinham como principal objectivo o estudo da estrutura visual do jacto, sem a obtenção de quaisquer resultados quantitativos [1, 6]. Estas investigações verificaram que a estrutura do jacto se alterava significativamente com o aumento da pressão. Primeiro a redução da tensão superficial leva à formação de ligamentos no jacto e de gotas de fluido que se separam da estrutura principal do jacto, um aumento maior da pressão da câmara de injecção para condições supercríticas conduz a uma estrutura do jacto semelhante àquela que 
seria visível numa injecção de um gás num ambiente também gasoso. Trabalhos experimentais mais recentes levaram a cabo estudos quantitativos nos quais foram obtidos resultados sobre o ângulo de abertura do jacto, densidade e temperatura [4, 7, 15, 16]. Estes resultados quantitativos experimentais permitem a comparação com os resultados obtidos em estudos numéricos e através da comparação é feita a validação destes mesmos modelos numéricos [17-22].

Barata et al. [18] realizou uma investigação inicial com o objectivo de avaliar as capacidades de um modelo computacional desenvolvido para escoamentos incompressíveis mas com densidade variável quando aplicado a condições supercríticas. Os resultados alcançados mostram ser concordantes com os dados experimentais, no entanto, nesta investigação apenas foram considerados razões de densidade intermédias de 0.05 a 0.14.

0 presente trabalho teve como principal objectivo estender a investigação da Ref. 18 a menores razões de densidade e definir qual o limite mínimo de razão de densidade para o qual se podia aplicar o modelo de escoamento incompressível mas com densidade variável. Para isso testaram-se diferentes razões de densidades abaixo de 0.05 . Testaram se as razões de densidade $\omega=0.010,0.015,0.020,0.025,0.035$ e 0.045 . Para estas razões de densidades obtiveram-se os campos escalares e da velocidade, gráficos da variação axial da densidade na linha central, do decaimento axial de velocidade na linha central e finalmente da metade da largura do meio da velocidade máxima (HWHMV) o qual permitiu determinar a tangente do ângulo de abertura do jacto.

A analise dos gráficos permitiu concluir que o modelo de gases de densidade variável resultados bastante concordantes com outros trabalhos para razões de densidades de $0.025 \mathrm{a}$ 0.045. No entanto definiu-se que o modelo deixa de ser aplicável para razões de densidade inferiores a 0.025 .

Um breve estudo, sobre a influência da velocidade de injecção sobre o comportamento obtido para o jacto no presente modelo, concluiu que a velocidade de injecção influência os resultados obtidos sobre o comportamento do jacto e desta forma deve ser um parâmetro a ter em conta. 


\section{Abstract}

The present work is devoted to study cryogenic nitrogen jets in high subcritical conditions. Fuel injection is one of the great challenges in engineering of diesel engines, gas turbines and rocket engines, combining in the last one also the injection of oxidizer. It is widely known that the increase of operation pressures and temperatures increases engine efficiency and reduces fuel specific consumption. Thus, it is a general trend in modern engines the operation in increasingly higher pressures. However at higher chamber pressures the injected fluids may experience ambient conditions exceeding the critical values. Several authors stated that at these conditions the injected fluids suffers a change of its properties, and the traditional twophase flow models cannot correctly predict the jet behavior at these conditions, thus new computational models are needed for these specific conditions. Barata et al. [18] performed a numerical investigation aimed to evaluate the applicability of an incompressible but variable density model in liquid jets under sub-to-supercritical conditions. The results achieved agree well with the experimental data but they only considered intermediate density ratios from 0.05 to 0.14 . The objective of the present work was to extend the investigation of Ref. 18 to lower density ratios from 0.025 to 0.045 which correspond to cases with subcritical chamber pressures. The obtained results agree well with the experimental and numerical data of Chehroudi et al. presented in Ref. 18. It was also found in this work that the computational model does not offers valid results for density ratios bellow 0.025 , thus identifying the limit of applicability of model.

\section{Keywords}

Fuel injection; critical point; supercritical conditions; rocket engines; diesel engines; density ratio; jets; variable density. 
Eduardo Luís Santos Farias Antunes 


\section{Index}

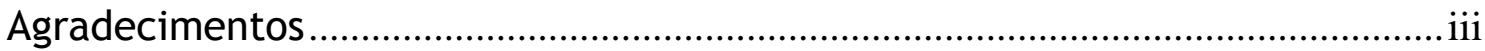

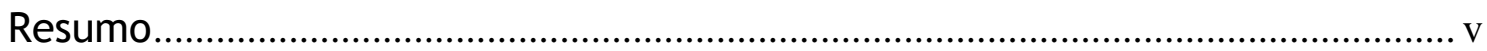

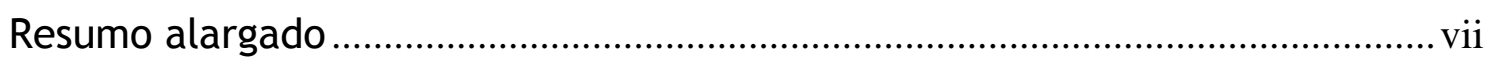

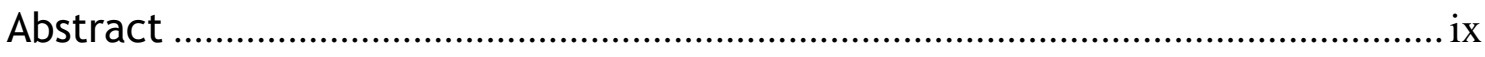

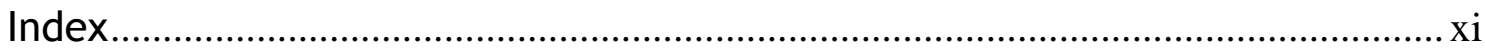

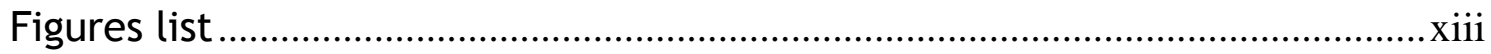

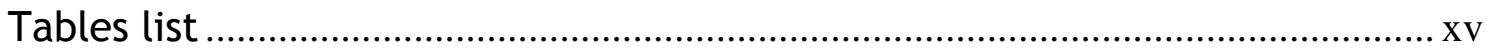

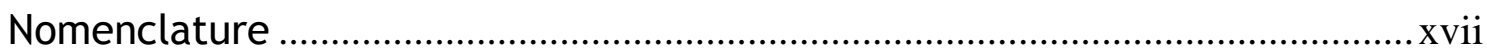

$X / D=$ axial distance normalized by injector diameter ...................................

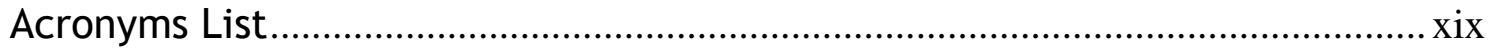

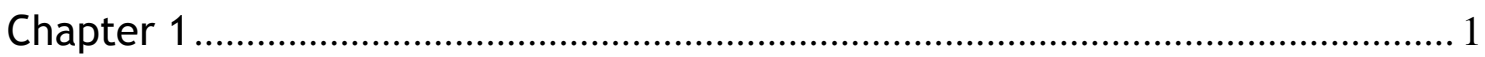

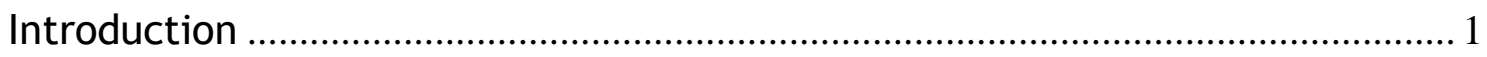

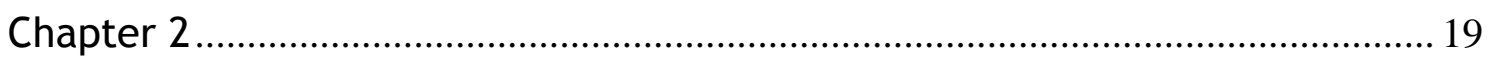

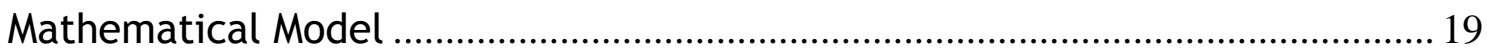

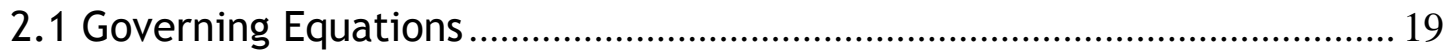

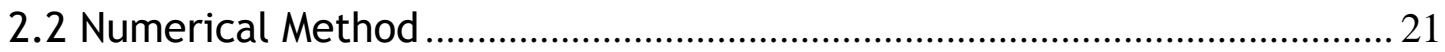

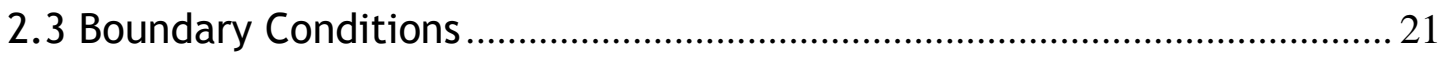

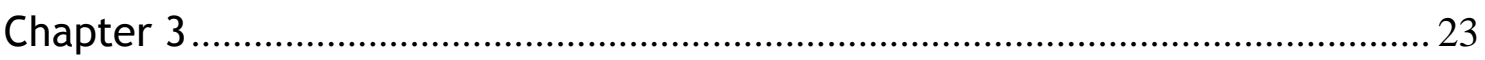

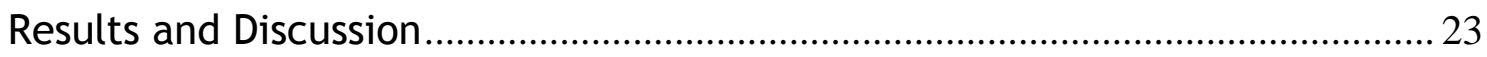

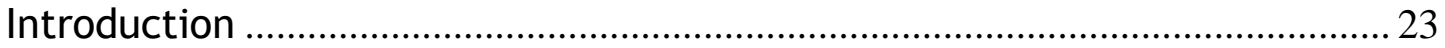

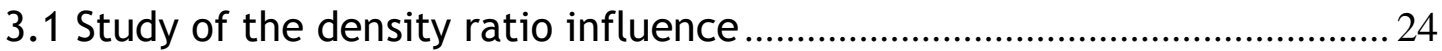

3.1.1 Velocity and scalar fields for $\omega=0.025,0.035$ and $0.045 \ldots \ldots \ldots \ldots . . .26$

3.1.2 Axial variation of centerline density for $\omega=0.025,0.035$ and 0.045

3.1.3 Centerline velocity decay for $\omega=0.025,0.035$ and $0.045 \ldots \ldots \ldots \ldots . . . . .37$

3.1.4 Half width of half maximum of the velocity for $\omega=0.025,0.035$ and 0.045

3.1.5 Centerline velocity and density decay rate at various chamber-toinjectant density ratios.

3.1.6 Tangent of the spreading angle at various chamber-to-injectant density ratios 
3.2 Study of the injection velocity influence.....................................................50

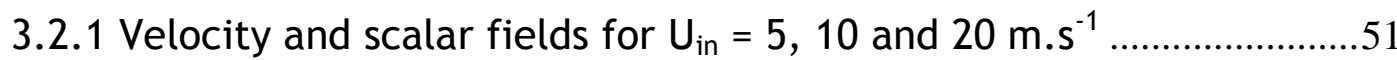

3.2.2 Axial variation of the centerline velocity for $U_{\text {in }}=3,5,10$ and 20 $\mathrm{m} . \mathrm{s}^{-1}$

3.2.3 Centerline velocity decay for $U_{\text {in }}=3,5,10$ and 20 m.s ${ }^{-1} \ldots \ldots \ldots \ldots \ldots . . . .56$

3.2.4 Half width of half maximum of the velocity for $U_{\text {in }}=3,5,10$ and 20 $\mathrm{m} . \mathrm{s}^{-1}$

Chapter 4

Conclusion. 61

References

Attachment 1

Attachment 2

Attachment 3 


\section{Figures list}

Figure 1- $\mathrm{LN}_{2}$ injection in $\mathrm{GN}_{2}$ at a) 4.0 , b) 3.0 and c) $2.0 \mathrm{MPa}$ [6].

Figure 2 - Software magnified images of the jet at its outer boundary showing the transition to the gas-jet like appearance, starting at just below the critical pressure of the injected fluid. Images are at a fixed supercritical chamber temperature of $300 \mathrm{~K}$ [1].

Figure 3 - Flow configuration

Figure 4 - Velocity and scalar fields of the jet with a density ratio of $\omega=0.025$ and a chamber pressure of $\operatorname{Pr}=0.583$, (a) Velocity vectors, (b) Mixture fraction contours, (c) Density contours. 26

Figure 5 - Velocity and scalar fields of the jet with a density ratio of $\omega=0.035$ and a chamber pressure of $\operatorname{Pr}=0.642$, (a) Velocity vectors, (b) Mixture fraction contours, (c) Density contours.

Figure 6 - Velocity and scalar fields of the jet with a density ratio of $\omega=0.045$ and a chamber pressure of $\operatorname{Pr}=0.825$, (a) Velocity vectors, (b) Mixture fraction contours, (c) Density contours. 30

Figure 7 - Axial variation of the centerline density with a density ratio of $\omega=0.025$ and a chamber pressure of $\operatorname{Pr}=0.583$.

Figure 8 - Axial variation of the centerline density with a density ratio of $\omega=0.035$ and a chamber pressure of $\operatorname{Pr}=0.642$.

Figure 9 - Axial variation of the centerline density with a density ratio of $\omega=0.045$ and a chamber pressure of $\operatorname{Pr}=0.825$.

Figure 10 - Axial variation of the centerline density in a logarithmic scale for the cases 1, 2 and 3.

Figure 11 - Centerline velocity decay for a density ratio of $\omega=0.025$ and a chamber pressure of $\operatorname{Pr}=0.583$. 37

Figure 12 Centerline velocity decay for a density ratio of $\omega=0.035$ and a chamber pressure of $\operatorname{Pr}=0.642$. 38

Figure 13 Centerline velocity decay for a density ratio of $\omega=0.045$ and a chamber pressure of $\operatorname{Pr}=0.825$. 39

Figure 14 - Centerline velocity decay for the cases 1,2 and 3 of Table 1. 41 Figure 15 - Half width of half maximum of the velocity for a density ratio of $\omega=0.025$ and a chamber pressure of $\operatorname{Pr}=0.583$.

Figure 16 - Half width of half maximum of the velocity for a density ratio of $\omega=0.035$ and a chamber pressure of $\operatorname{Pr}=0.642$.

Figure 17 - Half width of half maximum of the velocity for a density ratio of $\omega=0.045$ and a chamber pressure of $\operatorname{Pr}=0.825$.

Figure 18 - Half width of half maximum of the velocity for the cases 1, 2 and 3 of Table 1. . 46 
Figure 19 - Decay rate of the centerline velocity and density. ............................ 47

Figure 20 - Tangent of the spreading angle versus the chamber-to-injectant density ratio. .. 48 Figure 21 - Tangent of the spreading angle versus the chamber-to-injectant density ratio and representation if limit of applicability of the Variable Density approach................... 49 Figure 22 - Velocity and scalar fields of the jet with a density ratio of $\omega=0.025$ and an injection velocity of $U_{\text {in }}=5 \mathrm{~m} . \mathrm{s}^{-1}$, (a) Velocity vectors, (b) Mixture fraction contours, (c)

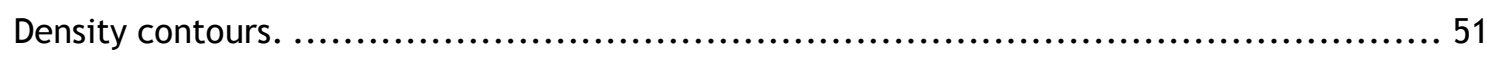

Figure 23 - Velocity and scalar fields of the jet with a density ratio of $\omega=0.025$ and an injection velocity of $U_{i n}=10 \mathrm{~m} \cdot \mathrm{s}^{-1}$, (a) Velocity vectors, (b) Mixture fraction contours, (c)

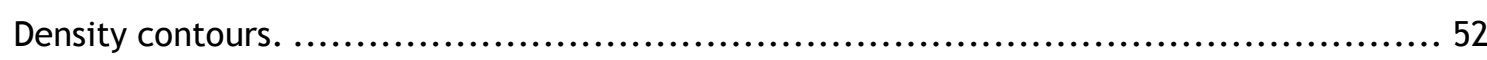

Figure 24 - Velocity and scalar fields of the jet with a density ratio of $\omega=0.025$ and an injection velocity of $U_{i n}=20 \mathrm{~m} \cdot \mathrm{s}^{-1}$, (a) Velocity vectors, (b) Mixture fraction contours, (c)

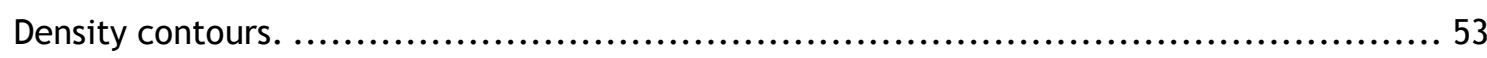

Figure 25 - Axial variation of the centerline density for the cases 4, 5, 6 and 7 of the Table 2.

Figure 26 - Centerline velocity decay for the cases 4, 5, 6 and 7 of the Table 2.......... 56 Figure 27 - Half width of half maximum of the velocity for the cases 4, 5, 6 and 7 of the Table 2. 58 


\section{Tables list}

Table 1. Summary of test conditions....

22

Table 2. Summary of test conditions for the injection velocity study 
Eduardo Luís Santos Farias Antunes 


\section{Nomenclature}

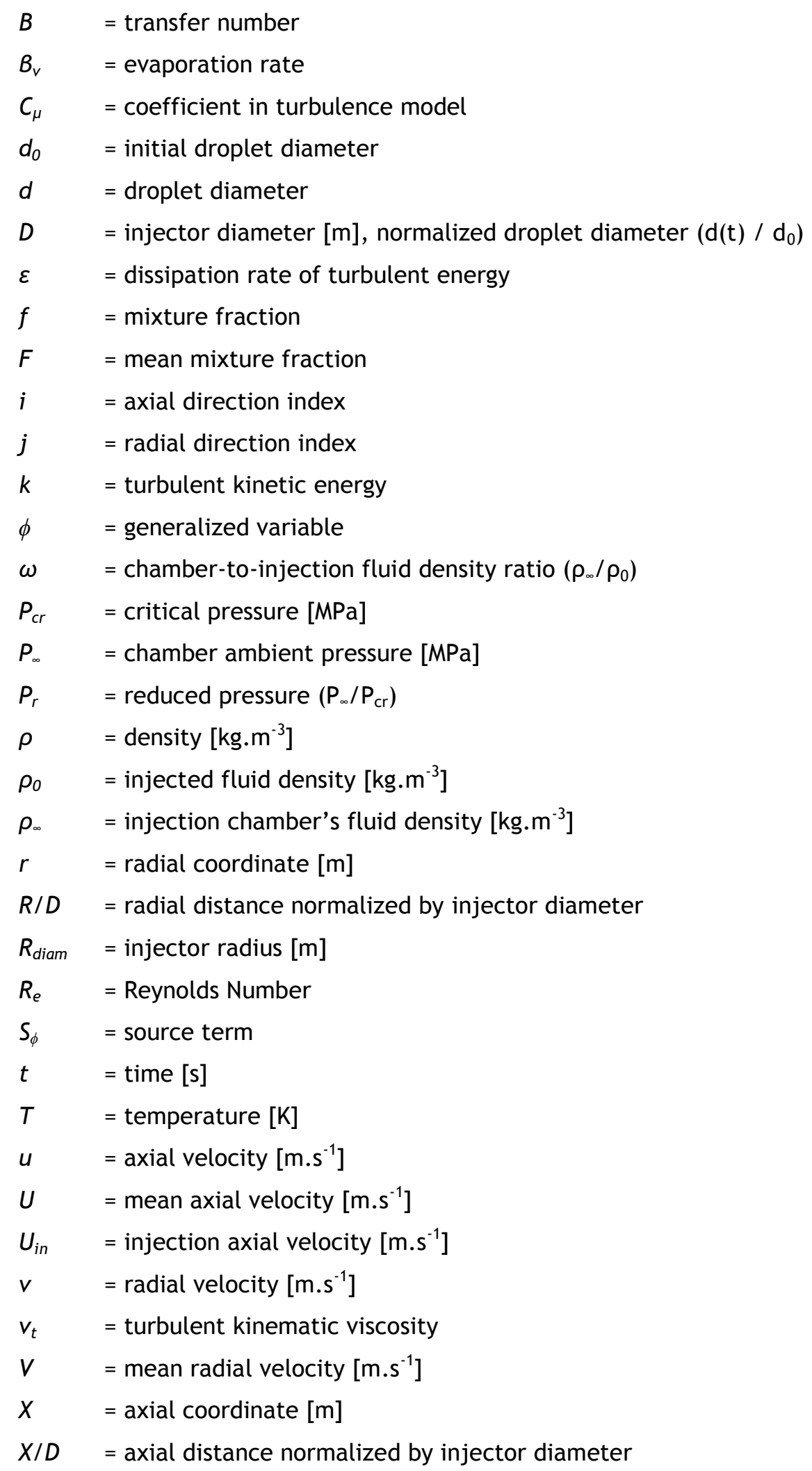


Eduardo Luís Santos Farias Antunes 


\section{Acronyms List}

NIST National Institute of Standards and Techonology

SPT

Standard conditions for temperature and pressure 
Eduardo Luís Santos Farias Antunes 


\section{Chapter 1}

\section{Introduction}

Fuel injection presents itself as one of the great challenges in engineering of diesel engines, gas turbines and rocket engines, combining in the last one also the injection of oxidizer. It is widely known that the increase of operating pressure and temperatures in combustion chamber, or thrust chamber in rocket engines, leads to an increase of engine efficiency, reducing this way the fuel consumption.

Thereby is a general trend in new engine designs to operate in increasingly higher chamber pressures and temperatures. Also the appearance of new and more resistant materials is other reason that could make grow this tendency. As a result of these increasingly higher pressures, the injected fluids may experience ambient conditions exceeding the critical values. As an example of particular interest in rocket engines, the liquid- $\mathrm{H}_{2}$ /liquid $-\mathrm{O}_{2}$ space shuttle main engine combustion chamber pressure is about 22.3 $\mathrm{MPa}$, while the combustion chamber pressure for the Vulcain engine (used on the Ariane 5) with the same propellants can reach up to a record value of $28.2 \mathrm{MPa}$ [1]. Both these pressure values are well above of the Hydrogen and Oxygen critical values, which for comparison are: $\mathrm{H}_{2}(32.94 \mathrm{~K} ; 1.28 \mathrm{Mpa})$ and $\mathrm{O}_{2}(154.6 \mathrm{~K} ; 5.04 \mathrm{Mpa})$. Also in diesel engines the combustion chamber can in some cases achieve a pressure twice the critical value. At these conditions the liquid fuel is on supercritical conditions and its physical state is named generally as "fluid" [2].

Several studies about the behaviour of fluid injection have been conducted and verified that the behaviour of liquids in critical, transcritical and supercritical conditions is not the same that is verified in more generic subcritical case, which was exhaustively treated and reported in existing, extensive literature. In this manner it seems of great interest the experimental and computational treatment of the supercritical case with the objective of understand, simulate and predict, in the most correct manner, the behaviour of injected fuel in surrounding environments where the pressure and temperature conditions are greater than the critical values for the injected fuel.

The main objective of the present work is to evaluate the performance of conventional computational methods for the prediction of single phase jets in supercritical conditions and the hypotheses of treating them as variable density gaseous jets instead of droplet sprays valid for subcritical conditions. This work is mostly dedicated for application on the analysis of the fuel injection process in liquid rocket engines.

In order to analyse the injection process in critical, transcritical and supercritical conditions is important to understand physical properties linked to these states and so, in the 
present revision will be given some emphasis to the understanding of behaviour of liquids and droplets evaporation prior to the treatment of the supercritical injection's itself.

Supercritical conditions are defined as the ones which the values of pressure or temperature, or even the junction of both, are greater that the critical values for a liquid or gas.

The critical point is described as a thermodynamic singularity, in this conditions the fluids characteristics suffers a significant change. The effective mass diffusivity, the surface tension and the latent heat of the liquid all vanish in critical conditions. On the other hand, the heat capacity at constant pressure, $C p$, the isentropic compressibility, $\mathrm{k}_{\mathrm{s}}$, and the thermal conductivity, $\lambda$, all become infinite [2]. The fact of the non existence of latent heat causes that, to vaporize the liquid no heat needs to be added, and thus, there isn't vaporization heat. This conclusion was also defended by Yang [3]. Bellan [2] considers that it is incorrect to talk about vaporization with values of pressure and temperature above the critical ones, and suggests the term of "emission". She also considers in his work that what truly defines the supercritical state is the impossibility of a two-phase region. In these conditions there is no separation between the liquid and the gas, and the substance should be named as fluid, as also argued by Segal and Polikhov [4]. Oschwald $M$ et al. [5] considers in their work that, in supercritical conditions, the addition of more heat does not conduct to a temperature augmentation, instead, all heat is converted in a rise of specific volume. An experimental work on injection of cryogenic fluids in hot fire conditions and could-flow conditions performed by Mayer et al. [6] concludes that increasing pressure at first reduces surface tension and a further increase in pressure through critical condition causes a complete vanish of the surface tension. Also the experimental work conducted by Chehroudi B et al. [7] reaches the conclusion that is evident the reduction of surface tension and enthalpy of vaporization as the critical pressure is achieved.

Another observation made by Bellan [2] is that the Navier-Stokes equations become increasingly invalid as the critical point is approached, and therefore any model of the critical/transcritical regime using the Navier-Stokes equations must be carefully scrutinized for inconsistencies.

In supercritical conditions, a clear bound between the injected liquid and the surrounding gas [4] vanishes, but the values of density to distinguish between injected liquid and the surrounding gas, it's of maximum relevance for supercritical models [5]. 
In the study of liquid droplets under supercritical conditions it is important to understand individually the effects of supercritical conditions in fluid properties by facilitating the distinction between the effects caused by supercritical conditions, and those due to other causes not necessarily linked to the supercritical state.

One of the most commonly studied cases for supercritical condition is the one of evaporation and emission of single liquid droplets. This kind of studies has the objective of determinate the processes that a liquid droplet encounter when mixing with the ambient gas and also to determine the mixing rate. Knowing the evaporation/emission rate of a liquid droplet is a very important information because this allows to determinate the droplet lifetime, the travelled distance for example in a combustion chamber, the time of the combustion and also the ignition delay, and all of this information is of the most relevance in the project of combustion chambers for engines and their respective injection systems.

Givler S.D. and Abraham J. [8] elaborated a revision about the several studies, conducted in the subjects of vaporization and droplets combustion under supercritical conditions, in which they describe the various conclusions achieved. One of the reached conclusions is that, despite its enormous importance for the modeling of combustion in sprays, until the paper's date (1996) there was not any published study about the interaction effects of multiple droplets in supercritical regime combustion. The same thing is not valid for the study of single droplets. A comparison is made in this work between the subcritical and the supercritical case. One of the conclusions achieved is that the droplet mixture process goes through two different stages. One initial transient stage in which the fluid droplet undergoes a temperature increase resulting from the fact of being in a higher temperature environment. The second stage of the process is one where the droplet temperature is constant and uniform, having reached the thermal equilibrium. At this stage the droplet is in quasi-steady regime at a temperature slightly below the boiling point and all the heat transferred to the droplet will be associated with the evaporation of more fluid quantity from the droplet.

These two stages described before are always seen in the subcritical case. It was also verified that in the subcritical case, especially under low pressure conditions, the transient stage has a significantly small duration when compared with the quasi-steady stage. At these conditions, by neglecting the transient stage, the droplet evaporation rate obeys to the square diameter evaporation law, often simply referred as " $D^{2}$ Law", and is correctly described by the next expression:

$$
d(t)^{2}=d_{0}{ }^{2}-\beta_{v} t
$$

Where $d_{0}$ is the initial droplet diameter, $t$ is the time and $\beta_{v}$ is the evaporation rate constant which may be shown to be: 


$$
\beta_{v} t=\frac{8 \rho_{g s} D_{g s}}{\rho_{l}} \ln (1+B)
$$

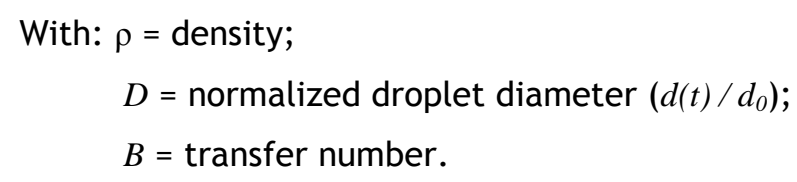

During these quasi-steady conditions the droplet is referred as being in a "wet-bulb state". The subcritical evaporation at low temperature is correctly described by the previous expression, as mentioned before. However, when ambient conditions approach critical values of pressure and temperature is observed that the transient stage suffers an increase in duration. In addition it is also observed that the evaporation begins to occur in this stage and not only in the quasi-steady stage. In the opposite to what happens in the quasi-steady stage, in the transient stage the droplet temperature is neither constant nor uniform and the $D^{2}$ Law can't be applied. Therefore, this law doesn't describe the first stage of the evaporation process and can only be used to describe the second stage. This leads to values increasingly deviating from the experimental values when ambient conditions approach the critical conditions. As a consequence, many works described by Givler and Abraham [8] focused on the study of evaporation/emission to the transcritical and supercritical cases. In this kind of studies it becomes important to distinguish between those performed in normal gravity conditions and others performed in micro-gravity conditions. The gravity is an important factor for the evaporation/emission studies because it is responsible for the buoyancy and convective effects. These effects have great influence in droplets evaporation/emission rate leading to its rise, and it becomes more difficult to conclude which physical phenomena are effectively responsible to an evaporation/emission rate variation and the way how it happens. Thus, it's interesting to conduct studies in microgravity conditions where the buoyancy and convective effects are minimized and it is easier to observe the direct changes in evaporation/emission rate caused by ambient pressure and temperature variations $[2,8]$.

With microgravity conditions Bellan [2] refers that higher pressures leads to a duration increase of the heating transient stage, which leads to a deviation from the $D^{2}$ linear model. In order to explain this deviation, several studies cited by Bellan [2] were performed with the objective of determining the droplet lifetime. These studies concluded that the temperature rise leads always to a decrease in the droplet lifetime. This is a logic and easy to understand conclusion, but the dependence of the droplet lifetime with pressure variations is not so easily understandable. It was found that the dependence relatively to the pressure varies 
with the temperature. So, for a temperature above about 1.2 times the critical temperature $\left(T_{r} \sim 1.2\right)$ the pressure rise leads to a monotonous decrease of the droplet lifetime. As for temperatures below about 0.8 times the critical pressure $\left(T_{r} \sim 0.8\right)$ the droplet lifetime increases with the pressure increase. Some authors also suggest that there might be a temperature at which the droplets lifetime is independent of pressure (see Bellan [2]). However, it should be pointed out that the studies done in microgravity conditions must be carefully analyzed since, even with gravity values as low as $10^{-2}$ in parabolic flights and $10^{-3}$ for experiences performed in drop towers, the buoyancy and convective effects are still present. Givler and Abraham [8] refer in their work that the experimental observations under conditions of microgravity are inconclusive, but their work was performed 5 years earlier that the one made by Bellan [2].

Zhang et al. [9] made a computational work using a numerical model that include the high pressure transient effects, temperature and pressure dependent variable thermo-physical properties in the gas and the liquid phases and the solubility of inert species in the liquid phase, for a moving $\mathrm{n}$-heptane droplet evaporation in a zero-gravity nitrogen environment. The unsteady equations of mass, species, momentum and energy conservation in axisymmetric spherical coordinates are solved using the finite-volume and SIMPLEC methods. The axisymmetric numerical model has been thoroughly validated against the extensive microgravity experimental data of Nomura et al. [10], in a work also referenced by Bellan [2] and Givler and Abraham [8].

In this work [10] it was noted that in high pressure environments the droplet is at a transient phase during all its lifetime, never reaching the quasi-steady phase of constant and uniform temperature. It was also verified that the increase of pressure is responsible for a decrease of droplet penetration distance and a rise in evaporation/emission rate.

As previously referred, studies of droplets evaporation/emission at normal gravity conditions have reported a problem of convection and buoyancy phenomena interference on the analysis of the direct effects of pressure and temperature on the evaporation/emission rate. However, smaller technical difficulties in the execution of these studies lead to more consistent results between different experiences. Studies reviewed by Bellan [2] indicate that for low pressure environments the emission rate obeys to the $D^{2}$ Law. However, as the pressure increases, it becomes more difficult to fit the obtained experimental results in the $D^{2}$ Law. It is known that the convection effects increase with the pressure and it becomes difficult to understand if the observed variation in emission rate comparatively to the $D^{2}$ Law is due to the thermodynamic mechanisms, to the fluids mechanic (through convective effects) or to the combination of both.

Givler and Abraham [8] also refer that Tsue et al. [11] had conducted one of the most remarkable experimental investigations about droplets supercritical vaporization, by achieving in all ambient conditions quasi-steady droplets vaporization. In agreement with previous studies, they concluded that the vaporization rate increases with ambient pressure. It is however concluded that the vaporization rate achieves a maximum and then decreases 
with further increases in ambient pressure. The experimental studies conducted with normal gravity by Givler and Abraham [8] agree that a higher ambient pressure corresponds to a vaporization rate increase. The final conclusions of the previous authors indicate that for subcritical and supercritical conditions with normalized pressure and temperature below to 2 , both transient and quasi-steady phases exist, indicating that for some supercritical conditions the quasi-steady model may be acceptable. However, for supercritical conditions where normalized pressure and temperature are above 2 all the emission process is made at the transient phase and in this situation the quasi-steady model is not applicable. Finally, it is concluded that for supercritical pressure and temperature the droplet lifetime decreases when the temperature increases.

A numerical investigation of $\mathrm{n}$-heptane droplet evaporation in nitrogen under transient and supercritical conditions, performed by Zhu and Aggarwal [13] reached similar conclusions. It was used a Lagrangian-Eulerian numerical method and were measured the density, latent heat, mole fraction, gas compressibility factor and the droplet lifetime. They concluded that droplet heat up time increases and becomes a more significant part of the droplet lifetime when the ambient pressure rises. As the droplet surface reaches a critical mixing point the latent heat of vaporization decreases and drops to zero. They also concluded that the droplet lifetime behavior it's not linear, and at low and moderate ambient temperature the droplet lifetime increases with pressure. However, at higher pressures, for temperatures of 500 and $700 \mathrm{~K}$, the droplet lifetime decrease with pressure. With higher temperatures the droplet lifetime also decreases with pressure. Finally Zhu and Aggarwal [13] concluded that when the droplet surface approaches the critical mixing state, the difference between the gas and liquid phases disappears.

Fieberg et al. [12] conducted an experimental and numerical work about fuel droplet evaporation under high pressure and temperature. They studied temperatures between $300 \mathrm{~K}$ and $500 \mathrm{~K}$ and the pressures between $0.1 \mathrm{MPa}$ and $3.7 \mathrm{MPa}$. For the experimental work the Phase Doppler Anemometry (PDA) technique was used and for the numerical work the FLUENT 6.0 CFD program. They measured the evaporation time, the surface temperature, the droplet diameter and the drag coefficient. It was also taken into account the effects of interaction between droplets in droplet chains. The conclusions reached in this work are that during the evaporation, the boundary layers increase because of the rapid diameter reduction and the exchange process between droplet surface and the adjacent gas phase slows down. Droplet deceleration in a droplet chain is much smaller compared to a single droplet, indicating that when injecting a group of droplets the penetration length should be much bigger than when injecting only one droplet. However, the burning rate of a single droplet is higher because of the existence of more available oxygen. For the experimental conditions the numerical results show that evaporation calculations in engine applications using quasi-steady modelling of the gas phase are valid even for supercritical conditions and produce acceptable errors compared to a fully transient calculation agreeing this way with the conclusions achieved by Givler and Abraham [8] that for some supercritical conditions the quasi-steady model is still 
valid. Since the evaporation takes place in a spray plume, the surrounding gas is cooled below the critical temperature and only a small number of droplets evaporate under supercritical conditions. The effect on the whole spray is thus further reduced.

The study of sprays and jet injection into gaseous environments at high pressure is considered as one of the most interesting subjects because it is the scientific basis for understanding the fuel injection process in diesel engines, gas turbines and rocket engines.

In this study it is given the jet denomination when the study of the disintegration of the fluid column injected is considered, the term spray is used when studying the path followed by the parcels of fluid (droplets) that have been separated from the injected fluid column [2]. In a spray there is always a separation process of the injected jet in several fluid parcels of different sizes which can occur in two different ways: by atomization or by disintegration. The former is a purely subcritical process, since it implies the existence of a superficial tension that must be broken. The last process only requires the existence of a boundary that hasn't necessarily to be a tangible surface. Hence, it is also concluded that the existence of a spray implies necessarily the existence of a separation between two phases, because only this way makes sense to speak of a boundary. So, the injection of a gas into an also gaseous environment will never lead to the existence of a spray.

The liquid jet injection in a gaseous environment under subcritical conditions represents a case of study treated by several authors and for which an extensive literature exists. Under subcritical conditions the injected liquid always has surface tension, meaning that the liquid will separate by atomization. When it contacts with the gaseous environment with a relative speed, the liquid will suffer the action of friction forces in excess of their surface tension and the surface segregation of liquid/gas will break down, resulting in smaller droplets which remain cohesive by creating a new surface in the gas separation. Due to their smaller surface/volume ratio, the smaller droplets momentum decreases faster when compared with the bigger ones. This implies that large droplets travel a greater distance [14]. Adding to this the fact that the evaporation process takes more time for bigger droplets, as these have a bigger transient phase and also a greater volume to evaporate, the travelled distance difference between the large and the small droplets is further amplified.

Several research teams investigated the liquid jet injection at diverse conditions ranging from subcritical condition to supercritical. These investigations are both in experimental as in numerical environments. The objective of these investigations is to determinate the influence of pressure and temperature in the jet behaviour.

Mayer et al. [6] conducted an experimental study on the injection of cryogenic fluids in hot fire (with combustion) and cold-flow conditions. The experiments were performed both for single jets as for coaxial jets. In the latter case the application was the injection of fuel and oxidizer on rocket engines. The chamber pressure employed for this study range from 1 $\mathrm{MPa}$ to $10 \mathrm{MPa}$. For the hot fire conditions only coaxial jets of gaseous hydrogen and liquid oxygen (LOX) were used. At these conditions, when ignited, the flow shows no droplets which 
is attributed to rapid vaporization and to the decrease of aerodynamic forces between the LOX and the $\mathrm{H}_{2}$ due to the reduction of density in the $3500 \mathrm{~K}$ hot reaction zone. It was expected that a decreased $\mathrm{H}_{2}$ injection temperature would lead to an increase of $\mathrm{H}_{2}$ density that would intensify the mixing process. However, it was found that increasing chamber pressure has a greater effect in intensified the mixing process, due to an increase in $\mathrm{H}_{2}$ density, than a decrease in $\mathrm{H}_{2}$ injection temperature. On cold-flow conditions both coaxial jets and singles jets were used. Liquid nitrogen was used instead of liquid oxygen, and the gaseous hydrogen was in some cases replaced by gaseous helium. The reason for these substitutions was to prevent combustion. The visualizations reveal that increasing the pressure reduces surface tension, which leads to an easier breakup of the jet and a formation of small droplets. It is also important to notice that the average diameter of formed droplets decreases as the ambient pressure rises, because the reduced surface tension is not strong enough to allow the existence of large droplets. A further pressure increase causes a complete vanish of the surface tension that leads to the disappearance of droplets formation. At Reynolds numbers exceeding 10,000 and for pressures higher than $70 \%$ of the critical pressure, the structure of the interface was dominated by turbulence at reduced surface tension, and did not depend on whether the pressure was supercritical or subcritical, when at a pressure of $6 \mathrm{MPa}$, fully under supercritical conditions, mixing is more like that between a dense and a light liquid in a turbulent shear layer and not more a liquid/gas mixing as in subcritical conditions. Finally, it was concluded that under certain conditions, often called transient conditions, the nature of the jet breakup process can be extremely sensitive to small perturbations in pressure, temperature, local mixture concentrations, and initial jet conditions, changing extremely fast from a liquid/gas behaviour to a gas/gas behaviour and vice versa. This study only reports qualitative results. Figure 1 shows the difference of the injection behaviour for supercritical, transcritical and subcritical conditions, evidencing the main conclusions reached in this work. 


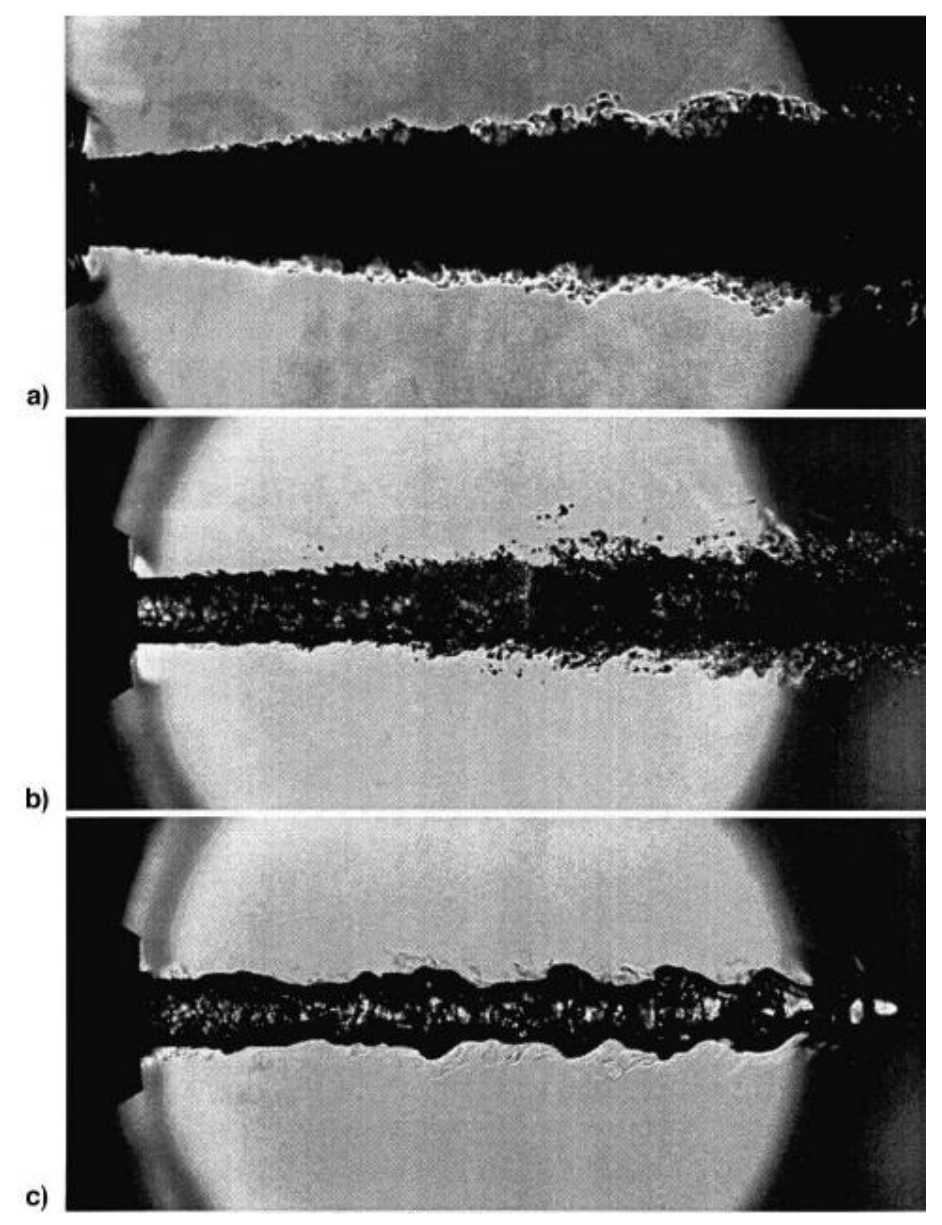

Figure 1- $\mathrm{LN}_{2}$ injection in $\mathrm{GN}_{2}$ at a) 4.0 , b) 3.0 and c) $2.0 \mathrm{MPa}$ [6].

Oschwald and Schik [15] made an experimental work injecting cryogenic nitrogen in a supercritical gaseous environment of nitrogen. Different pressures in the injection chamber of $4 \mathrm{MPa}$ and $6 \mathrm{MPa}$ were used. Both cases are supercritical, and correspond to normalized pressures of 1.17 and 1.76 respectively. The chamber temperature used was $298 \mathrm{~K}$ in all test cases and the injection temperature ranged from $100 \mathrm{~K}$ to $140 \mathrm{~K}$. Different injection temperatures of $5 \mathrm{~m} / \mathrm{s}$ and $20 \mathrm{~m} / \mathrm{s}$ were used. Using spontaneous Raman Scattering the authors were able to determine density and temperature data, and determined the density spreading angle and the temperature spreading angle. It was concluded that a supercritical fluid behaves like a boiling liquid. Heat transfer to an evaporating liquid at its boiling point results in further evaporation, but not in an increase of its temperature. This shows that there is a rise of the specific heat. In fact it was observed that under supercritical pressure the specific heat has a peak when close to the critical temperature. The increase of the injection temperature causes a faster decrease in the jet density. However at higher supercritical pressures the injection temperature has a smaller effect on the jet behaviour, and the jet is more stable. With lower injection temperatures the increase of the chamber pressure leads to a faster temperature increase. As far as the spreading angle is concerned it was concluded that initially (when the injection temperature is higher) the nitrogen is close 
to the critical temperature and the specific heat is very high, causing an initial smaller expansion and smaller spreading angle when compared with the cases with lower injection temperature. However, as the temperature rises, the case with a higher injection temperature departs from the critical temperature and the specific heat decreases leading to a higher expansion and higher spreading angle. In their work the authors also refer that in high pressure rocket engines the role of surface tension in the jet disintegration and mixing process is varying locally and equilibrium thermodynamics may be not adequate to describe the phenomena.

Chehroudi et al. [1, 7] also performed experimental studies of cryogenic jet injection under subcritical and supercritical pressures. In these studies quantitative results were obtained by measuring the jet growth rate/angle and the spreading rate of the mixing layer. They injected liquid $\mathrm{N}_{2}$, at a temperature between $99 \mathrm{~K}$ and $110 \mathrm{~K}$, in gaseous $\mathrm{N}_{2}$ at an ambient temperature of $300 \mathrm{~K}$. The critical pressure of $\mathrm{N}_{2}$ is $3.39 \mathrm{MPa}$ and the ambient pressures used in the studies range from the reduced values of 0.91 to 2.71 , the critical temperature of $\mathrm{N}_{2}$ is $126.2 \mathrm{~K}$. Using photography techniques, shadowgraphy and Raman scattering they were able to reach conclusions about supercritical injection, some of them agreeing with Mayer et al. [6]. Figure 2 reveals that when the chamber pressure is subcritical, the jet has a classic liquid-like appearance, but as the critical conditions are achieved the jet seems more like a gas/gas injection and the dark core of the jet appears to vanish and becomes less distinguishable from the ambient gas. These observations are validated by a quantitative agreement of the jet growth rate measurements with those predicted by the theoretical equations for incompressible variable-density gaseous jets, showing this way that besides having the appearance it has also the behaviour of a gaseous jet. Injection of liquid $N_{2}$ in gaseous $N_{2}$ at supercritical ambient temperature but at sub to supercritical pressure shows two structural transitions. One is when the intact jet with irregularly looking surface waves transforms into a diverging jet with ligaments and many small droplets, indicative of the second wind-induced atomization regime. The other is when the latter structure changes, not into the full atomization, but into a (single phase) gas/gas jet appearance slightly below the critical pressure. As pressure is increased, the jet width increases and the structure of the shear region changes from being dominated by ligaments and droplets to being dominated by finger-like structures, the major structural and interfacial changes occurred at $\operatorname{Pr}=1.03$. Above this $\mathrm{Pr}$, drops are no longer detected and regular finger-like entities are observed at the interface. They concluded that the change in the morphology of the mixing layer in supercritical conditions is evidently due to the combined effects of reductions in the surface tension and enthalpy of vaporization as the critical pressure is exceeded because of this transition. It was observed that at supercritical conditions the atomization regime is fully suppressed and they suggest that at these conditions cryogenic jet can indeed be treated like variable density gas jets as most of results obtained are similar to these ones. Finally, Chehroudi et al. [1] concluded that at critical and supercritical conditions, small changes in 
temperature at fixed pressure cause wide variations of density, thermal conductivity and mass diffusivity. This conclusion is also shared by Mayer et al. [6].

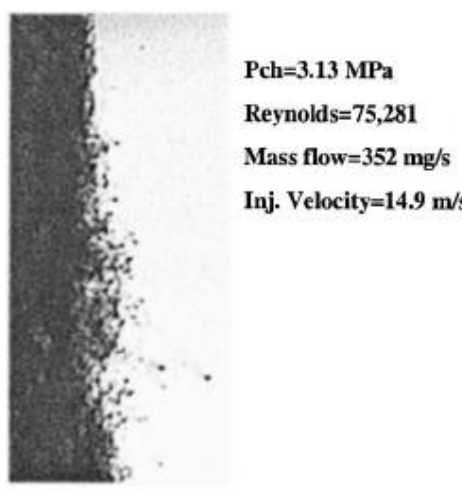

Appearance of conventional breakup of liquid surface indicating ligaments and drops ejecting from the mixing zone

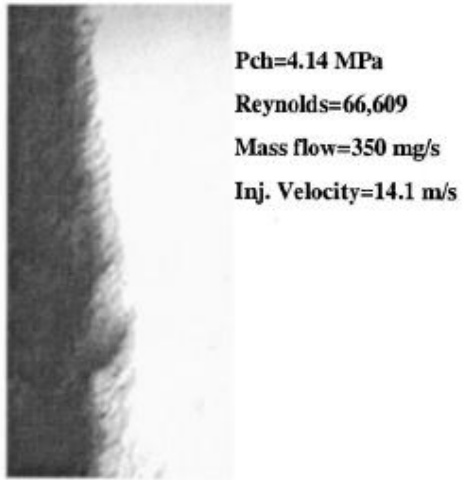

Mixing layer affected by sub- to supercritical transition. No drops are seen

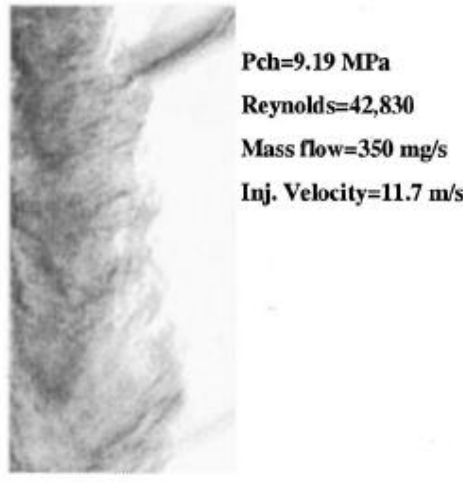

Appearance of
Gas/gas mixing
layer

Figure 2 - Software magnified images of the jet at its outer boundary showing the transition to the gasjet like appearance, starting at just below the critical pressure of the injected fluid. Images are at a fixed supercritical chamber temperature of $300 \mathrm{~K}[1]$.

Mayer and Telaar [16] have made one experimental work investigating the injection of cryogenic nitrogen in a gaseous nitrogen environment and the nitrogen/helium coaxial injection. Unlike the previous work of Mayer et al. [6] which only obtained qualitative results, this work obtained also quantitative results by the evaluation of the jet spreading angle using the shadowgraphy technique. For this experiment the injected nitrogen was at a temperature of approximately $100 \mathrm{~K}$ and the ambient gaseous nitrogen at a temperature of $300 \mathrm{~K}$. The chamber pressure used range from $1 \mathrm{MPa}$ to $6 \mathrm{MPa}$, encompassing this way both subcritical and supercritical pressure conditions. These authors firstly studied the nitrogen properties around the critical point. They conclude that at the critical point the densities of the gas and the liquid phase become the same, also the fluid properties vary remarkably around the critical point, with the specific heat experiencing a peak near this point and the surface tension decreasing as the pressure and temperature rise approaching critical values, this conclusion was also achieved by Chehroudi et al. [7]. The surface tension totally disappears when both pressure and temperature are supercritical and there is no longer phase equilibrium. However it's important to note that even above the critical pressure, surface tension is still present as long as the critical mixing temperature is not exceeded. The interpretation of the images obtain by the shadowgraphy technique in the cryogenic nitrogen injection led to the conclusion that at subcritical conditions the flow is controlled by aerodynamic and capillary forces, showing a wavy surface and droplet detachment. As the pressure rise in the subcritical range there are an increased number of droplets formed due to 
the decreased surface tension. At supercritical conditions shear forces exceed the capillary forces, which eventually disappear, and the flow start to be dominated by shear forces and the jet expansion, in fact in the trans- and supercritical regime, the surface tension plays only an initial role when the fluid is still below the critical mixing temperature. Finally, it was concluded that cryogenic jets in the supercritical regime, well above the critical point, show a purely gas-like behaviour.

Another experimental work on subcritical and supercritical liquid/gas mixing was performed by Segal and Polikhov [4]. In their work they injected a liquid into a gaseous nitrogen environment with reduced temperatures ranging from 0.68 to 1.28 and reduced pressures ranging from 0.2 to 2.2 , the injection speed ranged from $7 \mathrm{~m} / \mathrm{s}$ to $25 \mathrm{~m} / \mathrm{s}$ having the jet a Reynolds number between 11000 and 42000. The injected fluid chosen was FK-5-1$12\left[\mathrm{CF}_{3} \mathrm{CF}_{2} \mathrm{C}(\mathrm{O}) \mathrm{CF}\left(\mathrm{CF}_{3}\right)_{2}\right]$ for its good thermal stability, good spectroscopic properties, and the relatively low critical point, $p_{c}=18.4 \mathrm{~atm}, T_{c}=441 \mathrm{~K}$. It was used the technique of planar laser induced fluorescence (PLIF) to measure the density and the density gradients. By the analysis of the results it was concluded that surrounding gas inertia and surface tension forces dominate the mixing process in the subcritical case, pronounced ligament formation was observed at these conditions when the density gradient tends to be the highest. However, as transcritical conditions are achieved it is apparent a decrease importance of surface tension which manifests through the smoothening of the liquid-gas interface. Ligaments formation tends to significantly decrease. The ligament shape is similar to descriptions available in literature described before as finger-like structures or clusters. Packets of liquid continued to detach from the injected fluid and the density-gradient value decreased. With increased pressure and temperature above the critical conditions, the jet behaviour changed again: density-gradient values drastically decreased and approached values characteristic for a laminar jet at STP conditions, this type of a liquid/gas interface behaviour was described in previous computational results referred by authors but not seen in previous experiments. At supercritical conditions the mixing process is described as being controlled by vaporization rate of the injected fluid. The transitional mixing is defined as a domain when both subcritical and supercritical mixing types can be present, both droplet formation and irregularly shaped material broke from the injected fluid. This type of mixing is observed when only one thermodynamic variable exceeds its critical value or when either the pressure or the temperature was slightly below the critical value, thus supercritical mixing is possible even when only one thermodynamic variable exceeds its critical value.

It was observed in several experimental works that the processes which control the injection mechanism in the subcritical case are different from the ones that control it in the supercritical case. Thus it is important to find theories and computational models which can better predict the fluid behaviour under supercritical conditions. Several experimental works 
suggested that under supercritical conditions the liquid jet has a gas-like behaviour, this way, using variable density gas models to study supercritical jets is one possible approach to this problem. The variable density effects in axisymmetric isothermal turbulent jets for subcritical conditions were studied by Sanders et al. [17]. These authors used the standard " $k-\varepsilon$ " model and the second order Reynolds stress model with first- and second-order turbulence models to obtain velocities, dissipation rate, spreading rate, turbulence kinetic energy, velocity decay on the centreline and unmixedness and then compare the results obtain for both model with the experimental results obtain by other authors. The governing equation are solved using an algorithm similar to the TEACH code. It was concluded that the influence of varying density is not considered to be dependent on the precise value of the spreading rate, thus there is no significant influence of density ratio on the spreading rates in both models, as also observed in some experimental works. The influence of including buoyancy in the calculations with a density ratio above 1 is to decrease the spreading rates. However, without buoyancy it was observed a slower velocity decay and faster mixture fraction decay in the far field region. Finally the authors concluded that the " $k-\varepsilon$ " model cannot adequately simulate the buoyancy turbulence production and, although the differences are small, the Reynolds stress model showed better results than the " $k-\varepsilon$ " model. This work had not the aim of study supercritical conditions, but only the effects of variable density in turbulent jets.

A different hypothesis was formulated by Barata et al. [18]. Starting from the experimental evidence that supercritical jets behave as gaseous jets, they used the algorithm of Sanders et al. [17] to simulate sub- and supercritical conditions of a nitrogen jet injected in a nitrogen ambient. In their work they simulated the experimental conditions of Chehroudi et al. [7] with a chamber temperature of $300 \mathrm{~K}$ and an injection temperature ranging from $100 \mathrm{~K}$ to $110 \mathrm{~K}$. The chamber normalized pressure was ranging from 0.91 to 2.71 , thus having both subcritical and supercritical pressure conditions, and the injection velocity was ranging from $1.71 \mathrm{~m} / \mathrm{s}$ to $3.12 \mathrm{~m} / \mathrm{s}$. The results obtained in this work were the dense core length and thickness, velocity field and centreline velocity decay, scalar fields of density and mixture fraction, jet growth rate and the axial variation of centreline density. These results were then compared with theories and experimental work of other authors. It was observed that the length and thickness of the dense core decreases as the chamber pressure increases and approaches the values observed for a pure gaseous jet. Concerning the velocity decay along the centreline it increases when the normalized pressure increases. Despite the computational code was not written for the studied case, the numerical results of the spreading angle showed a good agreement with the experimental values obtained by Chehroudi et al. [7] and also with other theoretical works. However, when the density ratio decreases below the transition value, some small disagreements with experimental data are observed, since the jets approach the spray behaviour. This numerical work reached the conclusion that gaseous numerical models can indeed be used in the simulations of jets under supercritical conditions with a good degree of concordance. 
Zong et al. [19] performed a numerical work investigating cryogenic nitrogen injection and mixing into supercritical gaseous nitrogen. The nitrogen injection simulation was conducted through a circular duct with an inner diameter of $0.254 \mathrm{~mm}$. The injected cryogenic nitrogen was at a temperature of $120 \mathrm{~K}$ and gaseous nitrogen in the chamber was at a temperature of $300 \mathrm{~K}$ while the chamber pressure was ranging from $42 \mathrm{~atm}$ to $93 \mathrm{~atm}$. The fluid was injected at $15 \mathrm{~m} / \mathrm{s}$, as reference the critical pressure and temperature of nitrogen are $34 \mathrm{~atm}$ and 126 $\mathrm{K}$ respectively so all the tested cases are at supercritical conditions. The model used in this work accommodates full conservation laws, real-fluid thermodynamics and transport phenomena over the entire range of fluid states of concern. For turbulent closure was used a large-eddy simulation technique. This work was very comprehensive in terms of obtained results being presented the density and density gradient, specific heat, temperature, vorticity fields, velocity, kinematic viscosity, thermal diffusivity and other variables. An important conclusion this work was that due to the continuous variation of fluid properties through the jet and surrounding ambient, conventional treatment of fluid jets at low pressures in which the liquid and gas phases are solved separately and then matched at the interfaces often leads to erroneous results. The problem becomes even more exacerbated when the fluid approaches its critical state, around which fluid properties exhibit anomalous sensitivities with respect to local temperature and pressure variations. Thus, a prerequisite of any realistic treatment of supercritical fluid behavior lies in the establishment of a unified property evaluation scheme valid over the entire thermodynamic regime. The authors also claim that in the vicinity of the critical point a scaled equation of state must be used. Concerning the numerical results obtained for the fluid properties it was observed a sharp decrease of the density near the critical point as the temperature increases, it was also observed that the temperature sensitivity of the specific heat depends strongly on pressure. It increases rapidly as the fluid state approaches the critical point, and theoretically becomes infinite exactly at the critical point, this observation was also made in some experimental studies described before $[15,16]$. However, at very supercritical conditions $(9.4 \mathrm{MPa})$ the fluid shows a change in its behavior, the specific heat displays a very small variation through the critical temperature and the density shows a smooth transition as the temperature increases. The results obtained for the jet injection behaviour show the appearance of largescales instability waves near the injector in higher ambient pressures, also thread-like entities emerge from the jet surface at these pressures $(9.4 \mathrm{MPa})$. It was also seen that high ambient pressures facilitate the entrainment of ambient gaseous nitrogen into the cold jet fluid which increases the mixing process. Other reason for the increase of the mixing process in high ambient pressure is the decreasing of the density stratification at these pressures, the density stratification is responsible for inhibit of the formation of instability waves which accelerate the mixing process.

Sierra-Palares et al. [20] made a numerical investigation with the propose of assess turbulence models and discern which is the most suitable to use when dealing with supercritical fluids. Unlike the majority of the studies of this subject this work had not as 
motivation combustion chamber applications but the application in chemical reaction chambers. In this investigation a cryogenic nitrogen jet was injected into a pressurized reservoir of nitrogen at ambient temperature of approximately $300 \mathrm{~K}$ and at a pressure of 4 $\mathrm{MPa}$ was simulated. The cryogenic nitrogen was injected at a temperature ranging from $118 \mathrm{~K}$ to $140 \mathrm{~K}$ and with a velocity of $5 \mathrm{~m} / \mathrm{s}$, having this way a Reynolds number ranging from 115000 to 126000 . The objective of this work was to find the best model to deal with supercritical fluids, and several different " $k-\varepsilon$ " and " $k-\omega$ " turbulence models were used. In order to evaluate the performance of the various models used the dimensionless density and temperature profiles were compared between each other and with the experimental values. Also the residence time distribution results were compared between the models used and with other

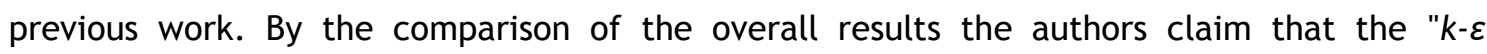
realizable" model is the one that shows the better results by having a deviation of approximately $16 \%$ from the experimental test cases while all the other models show are clearly less approximate with deviations above $20 \%$. Nevertheless, the authors consider that the other " $k-\varepsilon$ " models show also low deviations from the experimental results. The model could predict very well the residence time distribution even without any empirical information. However, the authors considered that because the actual turbulent models are not prepared to deal with the special characteristics of the supercritical turbulence, more improvements and adaptations are still needed in the model.

Another numerical work about jet injection was performed by Schimtt et al. [21]. These authors investigated the injection of a round nitrogen jet into a reservoir with supercritical pressure and also the injection of a coaxial hydrogen/oxygen jet with combustion. The objective of this numerical study was to build a real gas Large-Eddy Simulation solver that can compute large configurations with intricate geometries. Thus an unstructured explicit solver AVBP (which is a parallel CFD code that solves the three-dimensional compressible NavierStokes on unstructured and hybrid grids) was used to compute the compressible Navier-Stokes equations for a multispecies gas. The variables obtained in this work were the density and density field, the temperature and the $\mathrm{OH}$ mass fraction field, this last only for the investigation of the hydrogen/oxygen injection. In this work the authors describe the pseudoboiling temperature as the temperature at which, for a given pressure, the heat capacity at constant pressure reaches its maximum. Two cases with different injections temperatures were tested for the investigation of the nitrogen injection: the first case at a temperature slightly below the pseudo-boiling temperature while the second case was at a temperature slightly higher than the pseudo-boiling temperature. The pseudo-boiling temperature for this investigation was $129.5 \mathrm{~K}$ at a reservoir pressure of 39.7 bar. The injection temperature was $126.9 \mathrm{~K}$ for the first case and $137 \mathrm{~K}$ for the second case. One of the first conclusions achieved in this study was that at the pseudo-boiling temperature the specific heat experience a peak, this conclusion is also shared with previous experimental and numerical studies $[15,16,19]$. It was observed that in the vicinity of the critical temperature there is a strong dependence of the injection temperature, small changes in temperature cause big behaviour variations 
and this sensitivity to the temperature was also referred by other authors. The results for the flow visualisation showed that when the temperature is slightly below the pseudo-boiling temperature the jet shows ligament structures. However, for the second case of study, with the injection temperature only $10 \mathrm{~K}$ higher, the jet behaviour is completely different. The length of the dense core in the higher temperature case is smaller than in the case with lower injection temperature and jet growth angle is higher for the high injection temperature. Finally, it was concluded that the numerical results agree well with experimental data when the temperature is slightly below the pseudo-boiling temperature. Nevertheless, with a temperature slightly higher than pseudo-boiling temperature the numerical and experimental data show discrepancies.

Recently, Kim et al. [22] performed a numerical work about supercritical nitrogen injection. These researchers simulate the injection of liquid nitrogen with a temperature ranging from $128.7 \mathrm{~K}$ to $137.0 \mathrm{~K}$ in a chamber at a temperature of $278 \mathrm{~K}$ and a pressure ranging from 4.0 $\mathrm{MPa}$ to $5.98 \mathrm{MPa}$. The supercritical mixing processes were simulated by Large-Eddy Simulation based real-fluid models, the turbulence is generated by a "k- $\varepsilon$ " turbulence model and were used modified CHEMKIN packages. For this work were adopted a modified "Soave Redlich Kwong" (SRK) equation of state and a "Peng-Robinson" (PR) equation of state, both these equation were compared between each other and also with a model using an ideal-gas equation of state having as reference the experimental data achieved in previous works. The Reynolds number for this work ranged from 160000 to 200000 and the injector diameter was $2.2 \mathrm{~mm}$. Density, temperature, turbulent viscosity, turbulent kinetic energy, normalized axial velocity and specific heat were the obtain results used to establish the comparison between the models and the experimental result. By the analysis of the numerical results for the fluid properties, it was concluded that concerning the density the PR equation shows a better agreement with the NIST data in the higher pressure case, also in the cases with lower pressures and relatively low injection temperature the PR equation shows better agreement. The SRK equation shows a better agreement only in the case with lower pressure and relatively high injection temperature. Concerning the constant-pressure specific heat both equations of state agree well with NIST data for all temperature ranges except for a temperature of $129.5 \mathrm{~K}$ at near critical pressure. For the injection numerical results in the case with higher pressure the ideal-gas model erroneously underestimates the density level in the cold near-injector core region while the PR equation model slightly overestimate the density level in the same region, the SRK model is the more accurate, however for axial positions more downstream the PR equation starts to show a better agreement with the experimental data. In the case with lower chamber pressure and relatively lower temperature the PR equation has a better agreement with experimental data in both studied axial positions. For the case with relatively lower pressure but higher temperature the SRK equation shows a better agreement than PR equation. Finally, and agreeing with several other work it was conclude that near the critical pressure the fluid properties are very sensitive to small temperature variations it was also seen that when the pressure is equal to the critical 
value, there is a temperature where the constant-pressure specific heat becomes infinite, this temperature is called the as the pseudo-boiling temperature. If the pressure is slightly different from the critical value the pseudo-boiling temperature does not correspond to an infinite value of the constant-pressure specific heat but only to a maximum. At supercritical conditions, at the pseudo-boiling temperature, heat transfer to the nitrogen does not increase its temperature but merely increases its specific volume. It was also concluded that an increase in the pseudo-boiling strength might result in an increase of the cold core length.

In the present investigation, the work of Barata et al. [18] is extended to lower density ratios, corresponding to subcritical pressures, and the limits of application of the variable density approach to supercritical jets is investigated. 
Eduardo Luís Santos Farias Antunes 


\section{Chapter 2}

\section{Mathematical Model}

\subsection{Governing Equations}

The method to solve variable density jet flows is based on the solution of the conservation equations for momentum and mass. Turbulence is modeled with the “ $k \sim \varepsilon$ " turbulence model. A similar method has been used for three-dimensional or axisymmetric flows and only the main features are summarized here.

In the conservation equations, mass weighted averaging is applied to avoid the appearance of many terms involving density fluctuations for which additional models are needed. A mass averaged quantity is defined as

$$
\tilde{\phi}=\frac{\overline{\rho \phi}}{\bar{\rho}} .
$$

For the governing equations the standard parabolic truncation is employed. The mass averaged partial differential equations governing the steady, variable density axisymmetric flow may be written in cylindrical polar coordinates as

$$
\begin{gathered}
\frac{\partial \bar{\rho} U U}{\partial x}+\frac{1}{r} \frac{\partial r \bar{\rho} U V}{\partial r}=-\frac{\partial \bar{p}}{\partial x}-\frac{1}{r} \frac{\partial r \bar{\rho} \frac{\tilde{u^{\prime} v^{\prime}}}{\partial r}}{\partial \bar{\rho} U V} \\
\frac{\partial x}{r} \frac{1}{r} \frac{\partial r \bar{\rho} V V}{\partial r}=-\frac{\partial \bar{p}}{\partial x}-\frac{1}{r} \frac{\partial r \bar{\rho} \frac{\tilde{v^{\prime} v^{\prime}}}{\partial r}+\bar{\rho} \frac{\tilde{w^{\prime} w^{\prime}}}{r}}{}
\end{gathered}
$$

and the continuity equation as

$$
\frac{\partial \bar{\rho} U}{\partial x}+\frac{1}{r} \frac{\partial r \bar{\rho} V}{\partial r}=0
$$

To describe mixing of gases, the mixture fraction $F$, that represents the mass fraction of the nozzle fluid, is introduced. It obeys a convection-diffusion equation of the form 


$$
\frac{\partial \bar{\rho} U F}{\partial x}+\frac{1}{r} \frac{\partial r \bar{\rho} V F}{\partial r}=-\frac{1}{r} \frac{\partial r \bar{\rho} \frac{\tilde{\rho}}{v^{\prime} f^{\prime}}}{\partial r}
$$

In " $k \sim \mathcal{E}$ " turbulence model, the Reynolds stresses are expressed in terms of the local strain rate:

$$
-\bar{\rho} \overline{u_{i}^{\prime}{ }_{i}^{\prime}{ }_{j}}=\bar{\rho}\left(v_{t}+v\right)\left(\frac{\partial \tilde{u}_{i}}{\partial x_{j}}+\frac{\partial \tilde{u}_{j}}{\partial x_{i}}\right)-\frac{2}{3} \delta_{i j}\left(\bar{\rho} k+\bar{\rho}\left(v_{t}+v\right) \frac{\partial \tilde{u}_{j}}{\partial x_{j}}\right)
$$

with

$$
v_{t}=C_{\mu} \frac{k^{2}}{\varepsilon}
$$

The scalar flux in equation (7) is approximated with a gradient transport assumption

$$
\tilde{u_{i}^{\prime} f^{\prime}}=-\frac{v_{t}}{\sigma_{f}} \frac{\partial F}{\partial x_{i}}
$$

From the foregoing we can deduced the parabolized set of equations in cylindrical coordinates where the generalized equation is

$$
\frac{\partial}{\partial x}(\bar{\rho} U \tilde{\phi})+\frac{1}{r} \frac{\partial}{\partial r}(r \bar{\rho} \tilde{\phi})=\frac{1}{r} \frac{\partial}{\partial r}\left(r \bar{\rho} D \frac{\partial \tilde{\phi}}{d r}\right)+S_{\phi}
$$

where $\tilde{\phi}$ may stand for any of the velocities, turbulent kinetic energy, dissipation, or scalar property, and $S_{\phi}$ take on different values for each particular $\tilde{\phi}$, described in detail by Sanders et al [17].

The mean density can be obtained from the mean mixture fraction using the equation state. With constant pressure this leads to

$$
\frac{1}{\bar{\rho}}=\frac{F}{\rho_{o}}+\frac{1-F}{\rho_{\infty}}
$$

where density fluctuations have been neglected. This is allowed in isothermal jets because the instantaneous density, for which equation (12) is exact, is approximately a linear function of the instantaneous mixture fraction [18]. 


\subsection{Numerical Method}

The governing equations are solved using a parabolized marching algorithm which resembles the (elliptic) TEACH code [17]. The computations are performed by using the continuity equation to obtain the radial velocity $(V)$. Using the radial momentum equation for $V$ and solving a pressure correction equation for $V$ in radial direction did not show any difference with the use of the continuity equation [17]. In this approach the numerical model was applied to variable density jets and for the present case it was used for the study of liquid cryogenic jets under

In order to determine the tangent of the jet spreading angle is used the Half Width of Half Maximum of the Velocity (HWHMV), this routine has the function of determine the radial distance from the centerline $(r)$ at which the axial mean velocity $(U)$ is half of the maximum velocity localized in the centerline. This routine analysis all the points in the radial direction comparing them with half of the centerline velocity, when the routine finds a point bellow this value it saves the point before that.

\subsection{Boundary Conditions}

There are four boundaries in the computational domain, in these boundaries dependent variables are specified: an inlet and outlet plane, a symmetry axis and a free boundary parallel to the axis. The sensitivity of the solutions to the locations of the boundaries was investigated in previous works, and their final position is sufficiently far away from the jet so that the influence on the computed results is negligible [18]. At the inlet boundary the $1 / 7$ th power law turbulent velocity profile, represented in equation (13), was used for the axial velocity at the jet exit.

$$
U=U_{\text {in. }} \cdot\left(1-\frac{r}{R_{\text {diam }}}\right)^{\frac{1}{7}}
$$

The radial velocity is zero at the jet exit and in ambient. The mixture fraction is one at the jet exit and zero in the ambient. On the symmetry axis, the normal velocity vanishes, and the normal derivatives of the other variables are zero. At the outflow boundary, the gradients of dependent variables in the axial direction are set to zero.

The flow configuration can be observed in Figure 3. The injector nozzle has a diameter of $2.54 \times 10^{-4} \mathrm{~m}$ while the domain of study has an axial length of $1.77 \times 10^{-2} \mathrm{~m}$ and a radial length of $3.49 \times 10^{-3} \mathrm{~m}$. The test conditions for the present study of different densities ratios are presented in Table 1. 
It was also performed a parallel study with the objective of evaluate the numerical model for different injection velocities, the test conditions used are summarized in Table 2.

A minor modification was implemented in the outlet plane boundary of the mathematical model in order to avoid the velocity feedback which caused flow reversion at the outlet and was observed to happen at higher injection velocities. Thus a condition was imposed at the outlet that if near the outlet the axial velocity was negative, then the axial velocity at the outlet plane must be 0 .

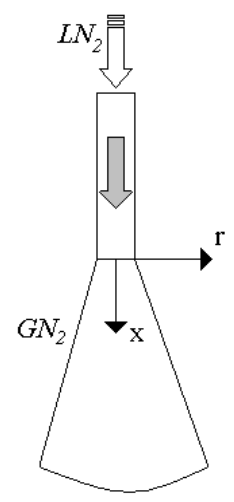

Figure 3 - Flow configuration

Table 1. Summary of test conditions

\begin{tabular}{|c|c|c|c|c|c|c|c|}
\hline Case & $\operatorname{Pr}$ & $\mathrm{U}_{\text {in }}[\mathrm{m} / \mathrm{s}]$ & $\operatorname{Re}$ & $\mathrm{T}_{0}[\mathrm{~K}]$ & $\begin{array}{c}\rho_{0} \\
{\left[\mathrm{Kg} / \mathrm{m}^{3}\right]}\end{array}$ & $\begin{array}{c}\rho_{\infty} \\
{\left[\mathrm{Kg} / \mathrm{m}^{3}\right]}\end{array}$ & $\omega$ \\
\hline 1 & 0,583 & 3.0 & 72060 & 100 & 700 & 17,5 & 0,025 \\
\hline 2 & 0,642 & 3.0 & 72060 & 100 & 700 & 24,5 & 0,035 \\
\hline 3 & 0,825 & 3.0 & 72060 & 100 & 700 & 31,5 & 0,045 \\
\hline
\end{tabular}

Table 2. Summary of test conditions for the injection velocity study

\begin{tabular}{|c|c|c|c|c|c|c|c|}
\hline Case & $\operatorname{Pr}$ & $\mathrm{U}_{\text {in }}[\mathrm{m} / \mathrm{s}]$ & $\operatorname{Re}$ & $\mathrm{T}_{0}[\mathrm{~K}]$ & $\begin{array}{c}\rho_{0} \\
{\left[\mathrm{Kg} / \mathrm{m}^{3}\right]}\end{array}$ & $\begin{array}{c}\rho_{\infty} \\
{\left[\mathrm{Kg} / \mathrm{m}^{3}\right]}\end{array}$ & $\omega$ \\
\hline 4 & 0,583 & 3.0 & 72060 & 100 & 700 & 17,5 & 0,025 \\
\hline 5 & 0,583 & 5.0 & 120100 & 100 & 700 & 17,5 & 0,025 \\
\hline 6 & 0,583 & 10.0 & 240200 & 100 & 700 & 17,5 & 0,025 \\
\hline 7 & 0,583 & 20.0 & 480400 & 100 & 700 & 17,5 & 0,025 \\
\hline
\end{tabular}




\section{Chapter 3}

\section{Results and Discussion}

\section{Introduction}

In this chapter the numerical results achieved in the present work will be presented and discussed.

The first subchapter includes the study of the influence of the ratio between the injected fluid density and the chamber pressure density in the several variables analyzed. The study was performed for three different density ratios, $\omega=0.025,0.035$ and 0.045 . For these 3 test cases were obtained results over the Half Width of Half Maximum Velocity (HWHMV) which was used to perform the calculation of the jet spreading angle. Results are shown about the axial variation of the centerline density and the centerline velocity decay for each studied case. In this chapter, the velocity field and the scalar fields of density and mixture fraction also presented and analyzed for all three cases. The results of the axial variation of the centerline density, centerline velocity decay and the half width of half maximum velocity are then compared between each other by the presentation, for each variable, of a graphic including the three studied cases. The results of the centerline density decay rate as well as the results of the centerline velocity decay rate are shown in a single graphic as a function to the density ratio.

Finally, the jet spreading angle results are condensed in a single graphic as a function of the density ratio, together with several other theoretical, experimental and numerical results. In this graphic is also shown the limit of application of the variable density approach.

The second subchapter presents the study of the influence of the injection velocity in the results achieved by the numerical model. In this study four different injection velocities, $U_{\text {in }}=$ $3,5,10$ and $20 \mathrm{~m} \cdot \mathrm{s}^{-1}$, were used with a density ratio of $\omega=0.025$. The axial variation of the centerline density, centerline velocity decay and half width of half maximum velocity were obtain for the four cases and compared between each other. Also the velocity and scalar fields of mixture fraction and are shown for the cases 5, 6 and 7 of Table 2 in this subchapter with the purpose of visualize the jet structure. The objective of this second part of the work was not to compare the obtained results with previous works, but instead to get a better understanding of the behavior of the numerical model. 


\subsection{Study of the density ratio influence}

The study of the density ratio influence in the injection behavior is the main objective of the present work.

Barata et al. ${ }^{18}$ performed an initial investigation aimed to evaluate the capabilities of a computational method developed for incompressible but variable density flows when applied to supercritical conditions. Their results have shown a good agreement with the experimental data, but they only considered intermediate density ratios from 0.05 to 0.14 . The present work extends the investigation of Ref. 18 to much lower density ratios and investigates the limits of application of the variable density approach to supercritical jets. For this objective the same computational model is used in sub-near critical conditions that are beyond the conditions for which the computational model was initially developed. In the present investigation several different density ratios were tested in order to define the limits of applicability of the numerical model. After the limit of applicability of the variable density model has been found, three different conditions were studied in detail within the range of applicability. The results achieved for these three conditions are shown in the present subchapter. The conditions outside the limit of applicability of the variable density model were not subjected to further studies, since it was verified that the numerical model does not work properly at those conditions.

Figures 4, 5 and 6 show the graphics of the velocity, mixture fraction and density fields for the density ratios of $\omega=0.025,0.030$ and 0.045 respectively. In these graphics the horizontal axis represents the axial distance to the injector $(X)$ while the vertical axis represents the radial distance to the centerline of the jet flow $(r)$. In the graphics of the velocity field, the velocity is represented by a vector for each point of the domain where the length of the vector represents the magnitude of the velocity. The mixture fraction and density scalar fields are represented by colored contours and with a color legend to allow the identification of the mixture fraction and density values in the graphic. The mixture fraction is zero when all fluid is chamber fluid and has the value 1 when is injected fluid. These graphics allow the visualization of the main structure of the jet.

Figures 7, 8 and 9 show the axial variation of the centerline density for the density ratios of $\omega=0.025,0.030$ and 0.045 respectively. The horizontal axis represents the axial distance to the injector, normalized by the injector diameter, while the vertical axis represents the density expressed in $\mathrm{kg} \cdot \mathrm{m}^{-3}$. These graphics allow the determination of the dense core length and this way helping to know the jet penetration. The Figure 10 shows the same results of Figures 7 to 9 but in the same graphic and in a logarithmic scale to facilitate the comparison between each other.

Figures 11,12 and 13 show the centerline velocity decay for the density ratios of $\omega=$ $0.025,0.030$ and 0.045 respectively. The horizontal axis represents the axial distance to the injector, normalized by the injector diameter, while the vertical axis represents axial velocity at the injector divided by the centerline axial velocity. These graphics, like the axial 
variation of the centerline density, is useful to determinate the jet penetration. The three obtained results are presented in the same graphic to allow the comparison between each other in the Figure 14.

The Half Width of Half Maximum Velocity (HWHMV) is shown in Figures 15, 16 and 17 for the density ratios of $\omega=0.025,0.030$ and 0.045 respectively. In these graphics the horizontal axis represents the axial distance to the injector while the vertical axis represents the radial distance to the centerline normalized by the injector diameter. These graphics show the radial distance at which the flow axial velocity is half the value of the axial velocity at the centerline. The point at which the axial velocity has this value is interpreted, in the present work, as the limit of the jet and is this way used to determine the jet spreading angle. To allow a better comparison between the results of the HWHMV the results of the three density ratios are shown in Figure 18.

Figure 19 shows the decay rate of the velocity and density in order to the chamber-toinjectant density ratio for the three studied cases. This graphic summarizes the conclusions also observed in graphics 7 to 14 .

Finally the Figure 20 shows the tangent of the spreading angle versus the chamber-toinjectant density ratio. This is the same graphic present by Barata et al. ${ }^{18}$ in their numerical work but with the inclusion of the six more density ratios studied in the present work, of this six studied density ratios only three are valid for the mathematical model and presented in detail in this subchapter. Thus, Figure 21 shows the same results that are presented in Figure 20 but without the three non valid results and with the inclusion of the representation of the variable density applicability limit. These graphics also allow the comparison between the results obtained in the present work and the results obtained by other authors. 


\subsubsection{Velocity and scalar fields for $\omega=0.025,0.035$ and 0.045}

a)

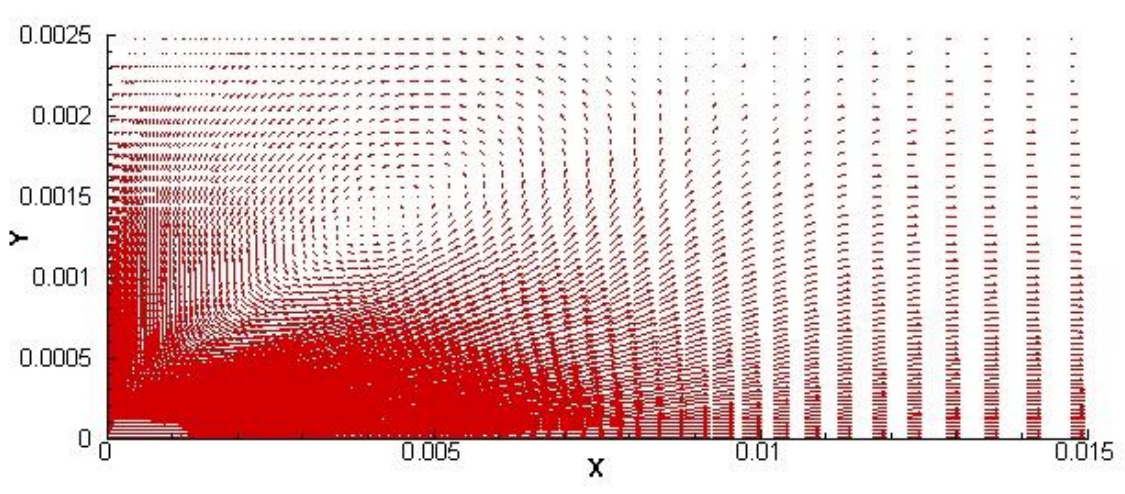

b)

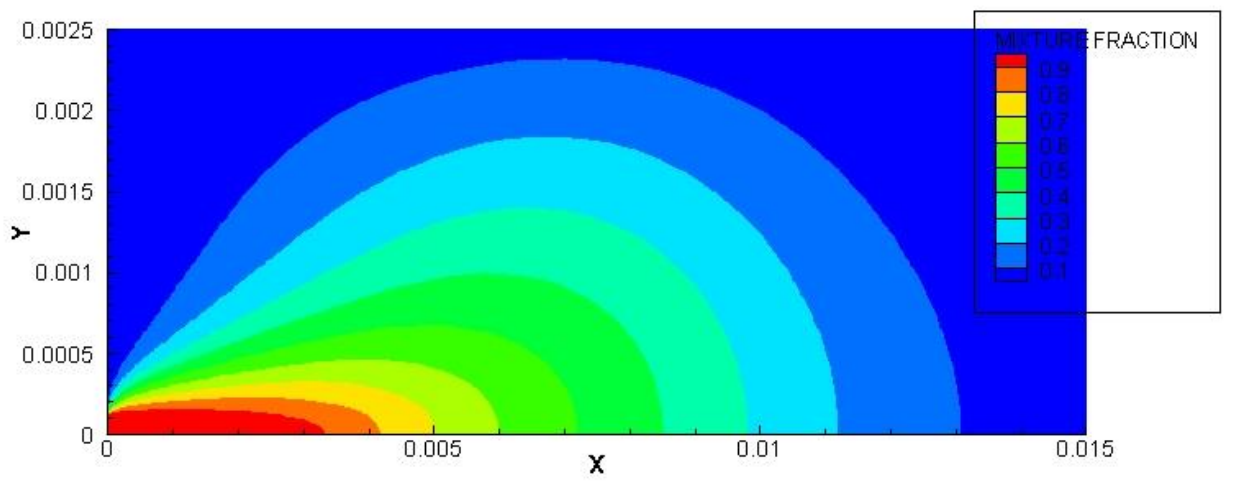

C)

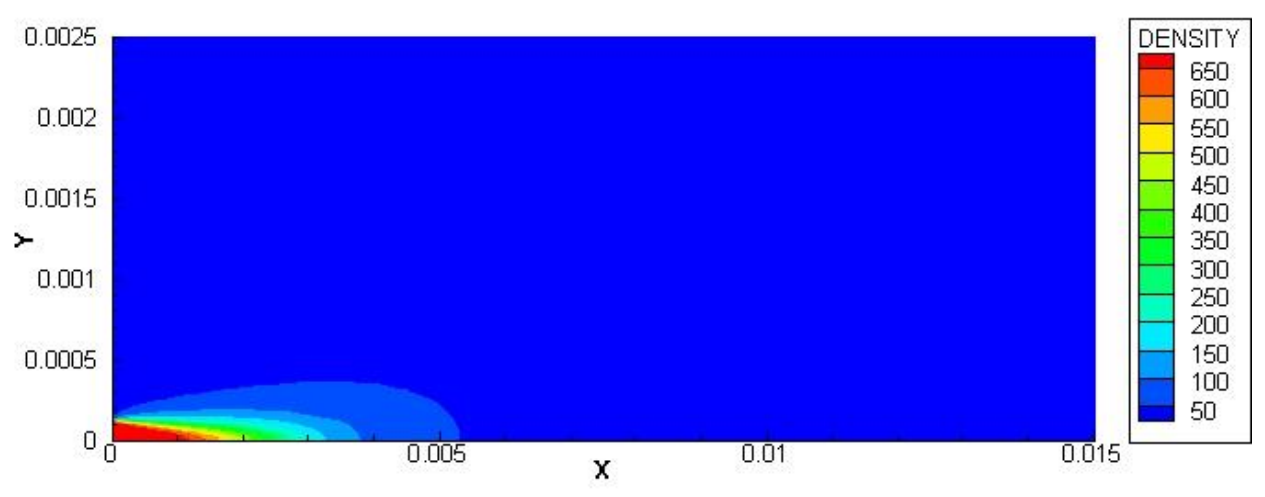

Figure 4 - Velocity and scalar fields of the jet with a density ratio of $\omega=0.025$ and a chamber pressure of $\operatorname{Pr}=0.583$, (a) Velocity vectors, (b) Mixture fraction contours, (c) Density contours. 
Figure 4 shows the velocity, mixture fraction and density fields for the Case 1 described in Table 1.

Figure 4-a) shows the velocity field within the studied domain. Close to the east boundary and at the injector exit it is observed that the velocity obeys to the 1/7 power law turbulent velocity profile having this way the maximum value at the centerline and then decreasing until the injector limit. The flow velocity above the injector nozzle shows a radial component of the velocity pointing at the centerline, this is due to the low pressures caused by the jet flow. As the jet progress it starts to drag the surrounding fluid giving it an axial component of the velocity. The two velocity components, imposed to the chamber fluid by the jet flow, are called as "entrainment" and, when combined, cause the formation of a vortex with the center localized approximately at an axial distance from the injector of $X=4.4 \mathrm{~mm}$ and a radial distance from the centerline of $Y=1.4 \mathrm{~mm}$. Downstream the of the center of the vortex there is a greater divergence in the jet velocity with an increment of the radial velocity towards the north boundary. After an axial length of approximately $X=10 \mathrm{~mm}$ the radial velocity starts to decrease as the flow leaves the vortex influence zone, and at a length of around $X=13 \mathrm{~mm}$ the flow has only axial velocity.

Figure 4-b) shows the mixture fraction scalar field for the domain of study. From the injector until an axial distance of around $X=3.3 \mathrm{~mm}$, close to the centerline, the mixture fraction has a value above 0.9 indicating that there was almost no mixture of the injected fluid with the chamber fluid. However the jet starts to mix with the chamber fluid in the radial direction, indicating an expansion of the injected fluid. Close to the centerline, at an axial length of approximately $X=7.2 \mathrm{~mm}$, the mixture fraction reaches a value of 0.5 which means that the injected fluid is fully mixed with the chamber fluid at this axial length.

The Figure 4-c) shows the density scalar field for the studied domain. It is observed a fast decrease of the density just after the injector. Close to the centerline the jet density goes from $700 \mathrm{~kg} \cdot \mathrm{m}^{-3}$ to $350 \mathrm{~kg} \cdot \mathrm{m}^{-3}$ in just approximately $\mathrm{X}=2.3 \mathrm{~mm}$. Comparing the density evolution with the mixture fraction evolution it is possible to conclude that while the mixture fraction at a axial distance of $X=3.3 \mathrm{~mm}$ at the centerline is still 0.9 , at the same length, the density close to the centerline is just $144 \mathrm{~kg} \cdot \mathrm{m}^{-3}$, this is, approximately $20.6 \%$ of the initial density. This means that the decrease of density is not caused by the mixture of injected fluid with chamber fluid, but instead caused by the large expansion of the injected fluid when within the injection chamber. The dense core (or dark core [4]), which can be defined as the jet central region of high density [4], has a very small length with just approximately $1.5 \mathrm{~mm}$, this result gives even more emphases to the rapid structural change experimented by the jet. 
a)

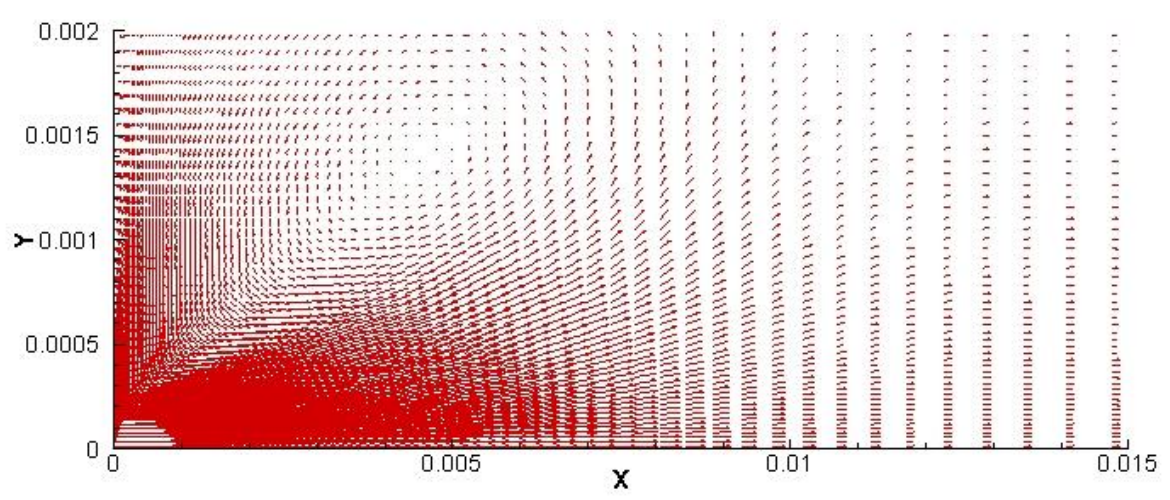

b)

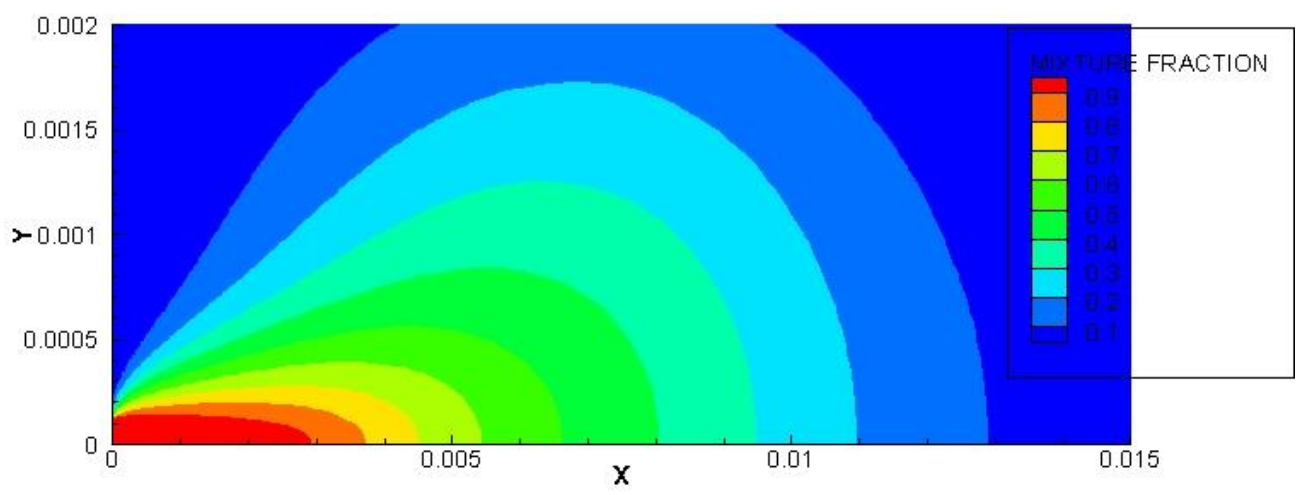

c)

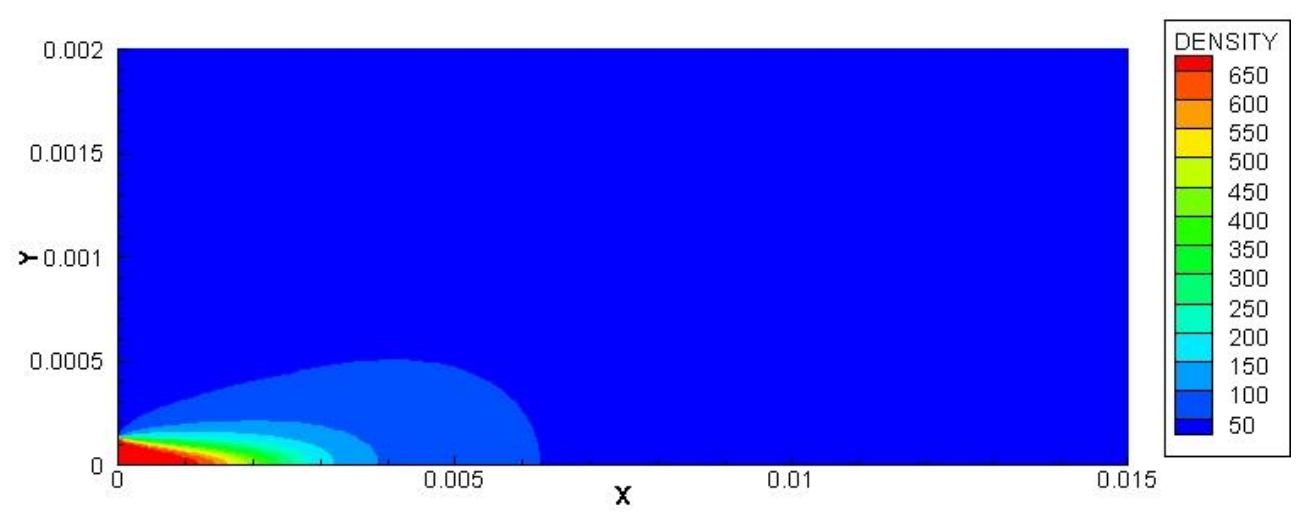

Figure 5 - Velocity and scalar fields of the jet with a density ratio of $\omega=0.035$ and a chamber pressure of $\operatorname{Pr}=0.642$, (a) Velocity vectors, (b) Mixture fraction contours, (c) Density contours. 
Figure 5 shows the velocity, mixture fraction and density fields for the Case 2 described in Table 1.

Figure 5-a) shows the velocity field within the studied domain. The velocity field for this case has the same aspect that the one in the case presented before. Thus, like in the previous case, also in this case is observed the entrainment of the surrounding fluid in the direction of the jet, this causes the increase of radial velocity towards the centerline and the increase of the axial velocity in the jet direction close to the east boundary, this ultimately origin the formation of a vortex. However in this case the center of the vortex is localized downstream the localization of the previous case, at an axial distance from the injector of $X=4.8 \mathrm{~mm}$ while approximately the same radial position. Downstream the vortex center is also observed a divergence of the velocity with it earning a radial component toward the north boundary. This radial component eventually ceases at an axial distance from the injector of approximately $X=11 \mathrm{~mm}$ and the velocity retains only its axial component.

Figure 5-b) shows the mixture fraction scalar field for the domain of study. The mixture fraction shows a rapid decrease across the jet progression. Following the centerline the mixture fraction remains above 0.9 until an axial distance to the injector of $X=2.9 \mathrm{~mm}$, this means that until this distance the jet fluid practically does not mixture with the ambient fluid. The point of equal composition between the injected fluid and the chamber fluid (mixture fraction equal to 0.5 ) is reached, in the centerline, at an axial distance to the injector of $X=6.6 \mathrm{~mm}$. Comparing with case 1 this case shows a faster mixing process as the same mixture fraction values are obtain upstream of the localization found for case 1.

Figure 5-c) shows the density scalar field within the domain of study. The density shows an even faster decrease along the jet progression than the mixture fraction. While the mixture fraction has a decrease of $50 \%$ along the centerline in $X=6.6 \mathrm{~mm}$, the density decreases to half in just approximately $X=2.2 \mathrm{~mm}$. Again, this shows that the decrease of the density value is caused mainly by the jet expansion and not by the mixture with the ambient fluid. The dense core for this case has a length of approximately $1.2 \mathrm{~mm}$ which is smaller than in the previous case. It can be conclude that in this the jet suffers a larger expansion that in the case 1. 
a)

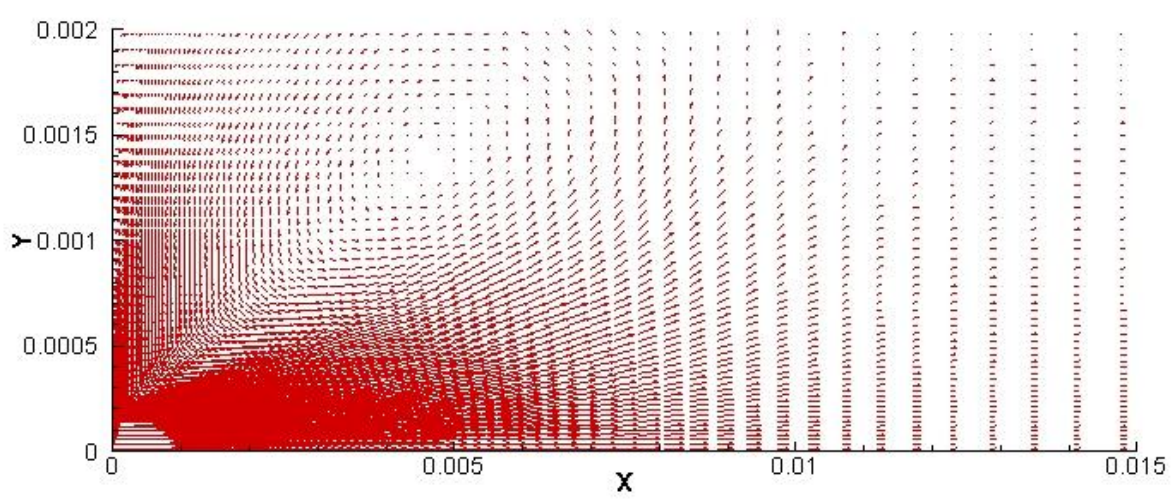

b)

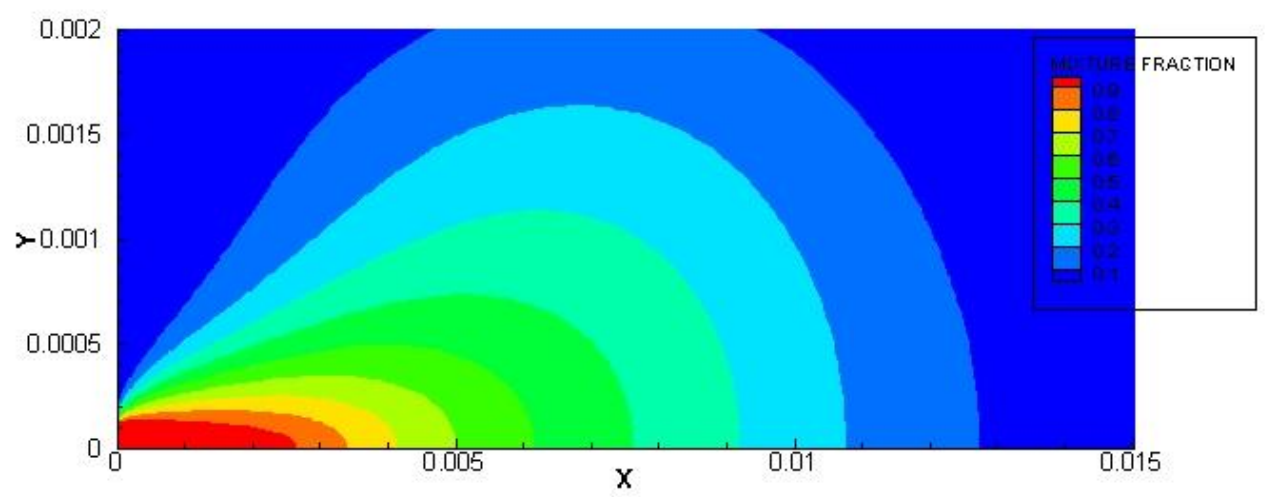

c)

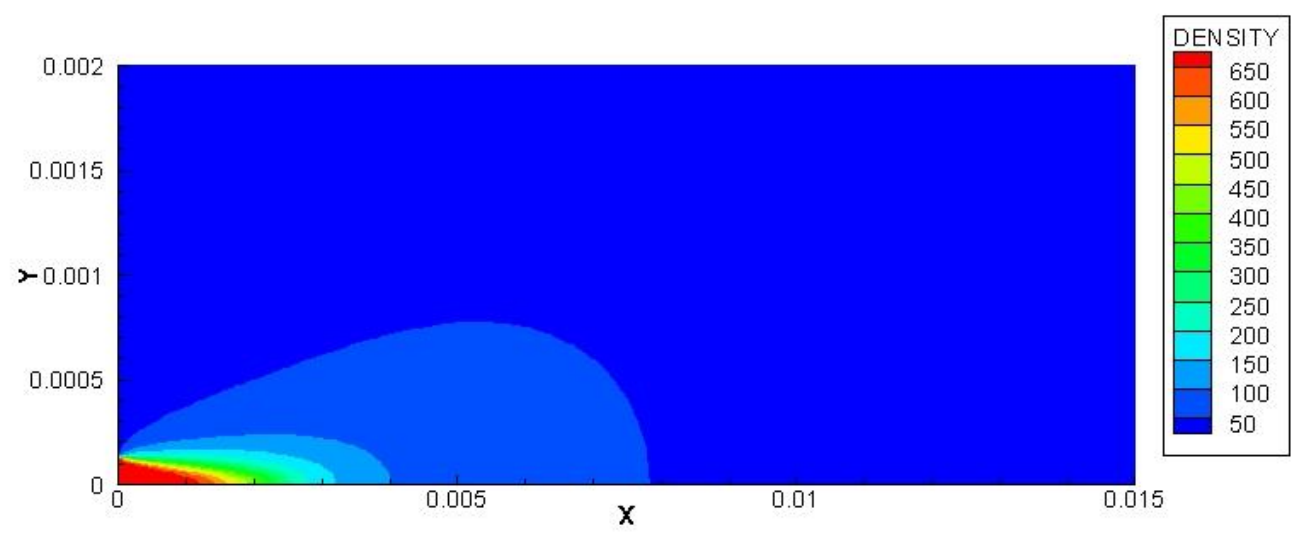

Figure 6 - Velocity and scalar fields of the jet with a density ratio of $\omega=0.045$ and a chamber pressure of $\operatorname{Pr}=0.825$, (a) Velocity vectors, (b) Mixture fraction contours, (c) Density contours. 
Figure 6 shows the velocity, mixture fraction and density fields for the Case 3 described in Table 1.

Figure 6-a) represents the velocity field within the studied domain. It is observed like in other cases that the ambient fluid next to the east wall and above the injector gains a radial component of velocity towards the jet centerline ("entrainment" phenomena). The surrounding fluid is also entrained in the direction of the jet and a vortex appears with its center at an axial distance from the injector of $X=4.8 \mathrm{~mm}$ and a radial distance from the centerline of $Y=1.4 \mathrm{~mm}$, approximately the same position that it was on case 2 . The jet velocity then gains a radial component towards the north wall after the center of the vortex. This radial component of the velocity starts to decrease at an axial distance of approximately $X=8 \mathrm{~mm}$ and eventually becomes close to zero at an axial distance of approximately $X=11$ $\mathrm{mm}$.

The Figure 6-b) shows the mixture fraction scalar field for the domain of study. The jet mixture fraction shows a rapid decrease along the centerline. In this case the jet remains almost unmixed (with a mixture fraction value above 0.9) until an axial distance of $X=2.6$ $\mathrm{mm}$ and then it starts to mix with the ambient fluid reaching a 0.5 mixture fraction on the centerline at an axial distance of approximately $X=6.1 \mathrm{~mm}$. In the centerline, at an axial distance to the injector of approximately $X=12.7 \mathrm{~mm}$, the mixture fraction is below 0.1 indicating that after this point injected fluid is fully dispersed into the ambient fluid, comparing this with the velocity field at the same distance, can be stated that the jet does not exist anymore after this point. Comparing with the previous cases, it is possible to conclude that for this case the mixture fraction decreases faster than in previous studied cases with lower chamber pressures.

Figure 6-c) shows the density scalar field within the domain of study. The jet shows a very fast decrease of the density along the centerline with the value of the density in the centerline decreasing by half in less than $2.2 \mathrm{~mm}$ from the injector. When compared with the mixture fraction which is half of its initial value at an axial distance from the injector of $X=$ $6.1 \mathrm{~mm}$, this show that the density decrease of the jet fluid is not caused by the mixing of the jet fluid with the less dense ambient fluid, because at the point that the density is half the initial density the mixture fraction is still above 0.9 indicating that almost no mixing with the ambient fluid has already happen at this point. So the decrease in density must be caused by the rapid expansion of the jet fluid as it enters in the injection chamber. The jet dense core in this case has a length in the centerline of approximately $1.2 \mathrm{~mm}$.

Comparing the velocity and scalar fields for the three studied cases it is possible to conclude that in general the jet behavior is very similar between each other. This was expected because all the test cases are in the subcritical range with just only slightly increments of pressure from case to case. However, some differences can be noted between the test cases, for example in the velocity field is observed that at an axial length of $X=15$ $\mathrm{mm}$ the flow has a greater velocity for the case with lower pressure and density ratio (case 1 ) 
while the flow with lower velocity is the case with higher pressure and density ratio (case 3), this could indicate a greater interaction between the injected fluid and the ambient fluid in the case with higher pressure. Analyzing the graphics of the mixture fraction scalar field it is observed that at higher density ratios the same mixture fraction values are obtained more upstream, this indicates that for conditions closer to the critical point the mixing process between the injected and the ambient fluid is amplified, this conclusion is in order to the conclusions found in the majority of experimental and numerical works performed previously and also reinforces the idea of a greater interaction between the fluids at higher density ratios. The comparison of the small differences between the density scalar fields for the three cases also allows some conclusions. At higher pressures and density ratios the density shows a faster decrease. As stated previously, the density decrease is consequence of the jet expansion, thus for higher density ratios the injected fluid shows a faster expansion. As the injected fluid is initially in liquid state (and liquids are viewed as incompressible), in a real injection this means that, to expand, the liquid must be firstly warmed into a gaseous state, and then starts the expansion. Bellan [2] refers in her work that the evaporation of a single droplet of fluid is faster as the pressure increases and approaches the critical value. This allows the deduction that greater pressures also cause faster expansions of the fluid. Thus, even without the consideration of the phase transition by the present model, the faster expansion at higher pressures near the critical pressure is verified in the obtained results. 


\subsubsection{Axial variation of centerline density for $\omega=0.025,0.035$ and 0.045}

The Figure 7 shows the axial variation of the centreline density for the Case 1 described in Table 1.

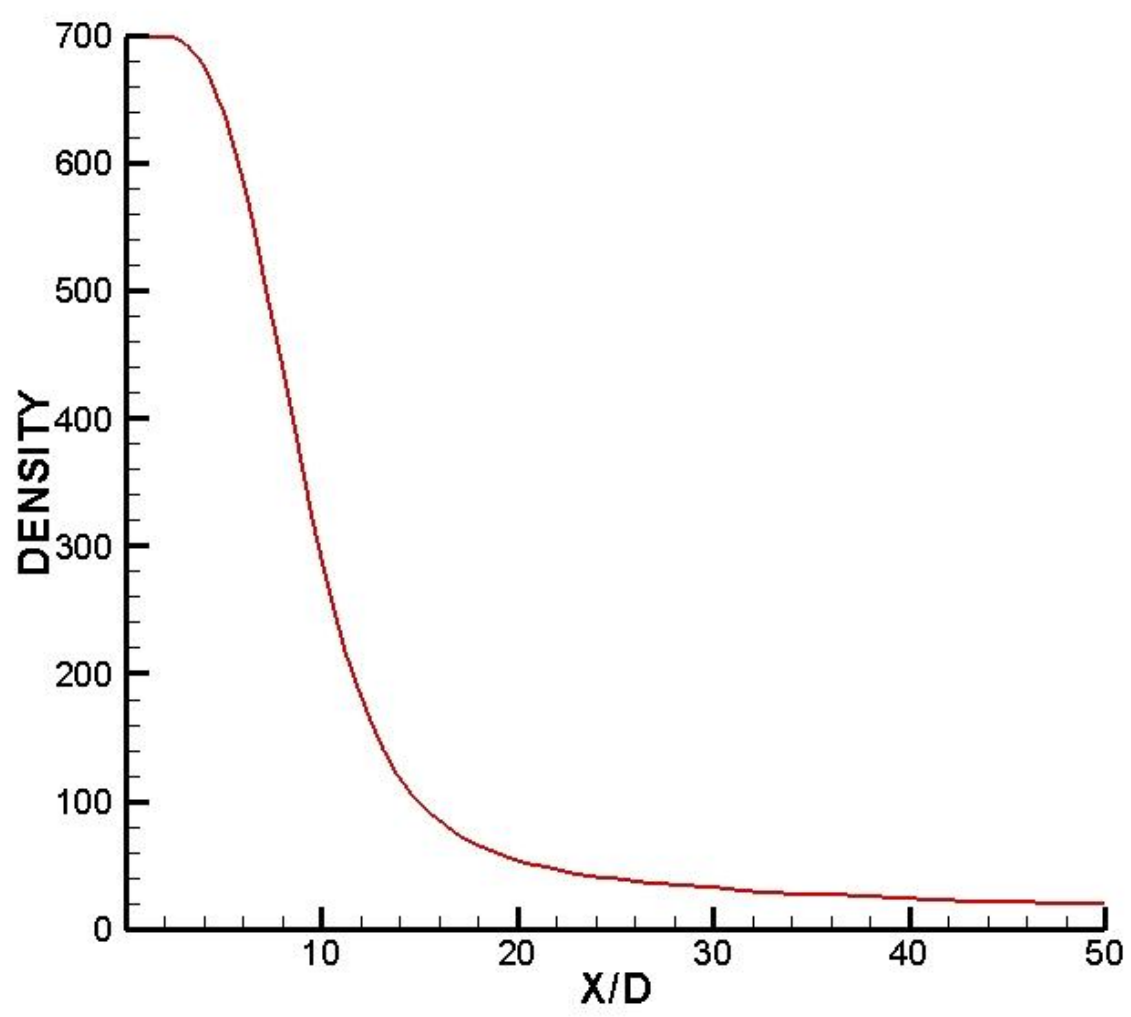

Figure 7 - Axial variation of the centerline density with a density ratio of $\omega=0.025$ and a chamber pressure of $\operatorname{Pr}=0.583$.

The density remains approximately its initial value of $\rho=700 \mathrm{~kg} \cdot \mathrm{m}^{-3}$ until an axial distance of $X / D=3$ diameters, which corresponds to the potential core. After this point, the density starts an accentuated decrease which corresponds to the jet rapid expansion. The density decrease starts to slows down at an axial distance of $X / D=15$ diameters, at this point the centerline density has a value of approximately $\rho=100 \mathrm{~kg} \cdot \mathrm{m}^{-3}$. After this point the centerline density decreases at a slower rate, reaching a value of around $\rho=20 \mathrm{~kg} \cdot \mathrm{m}^{-3}$ at an axial distance of $X / D=50$ diameters. Comparing this graphic with Figure $4-c$ ) it is possible to see that the start of the accentuated decrease of the centerline density is close to the point at which the jet dense core disappears. 
The Figure 8 shows the axial variation of the centreline density for the Case 2 described in Table 1.

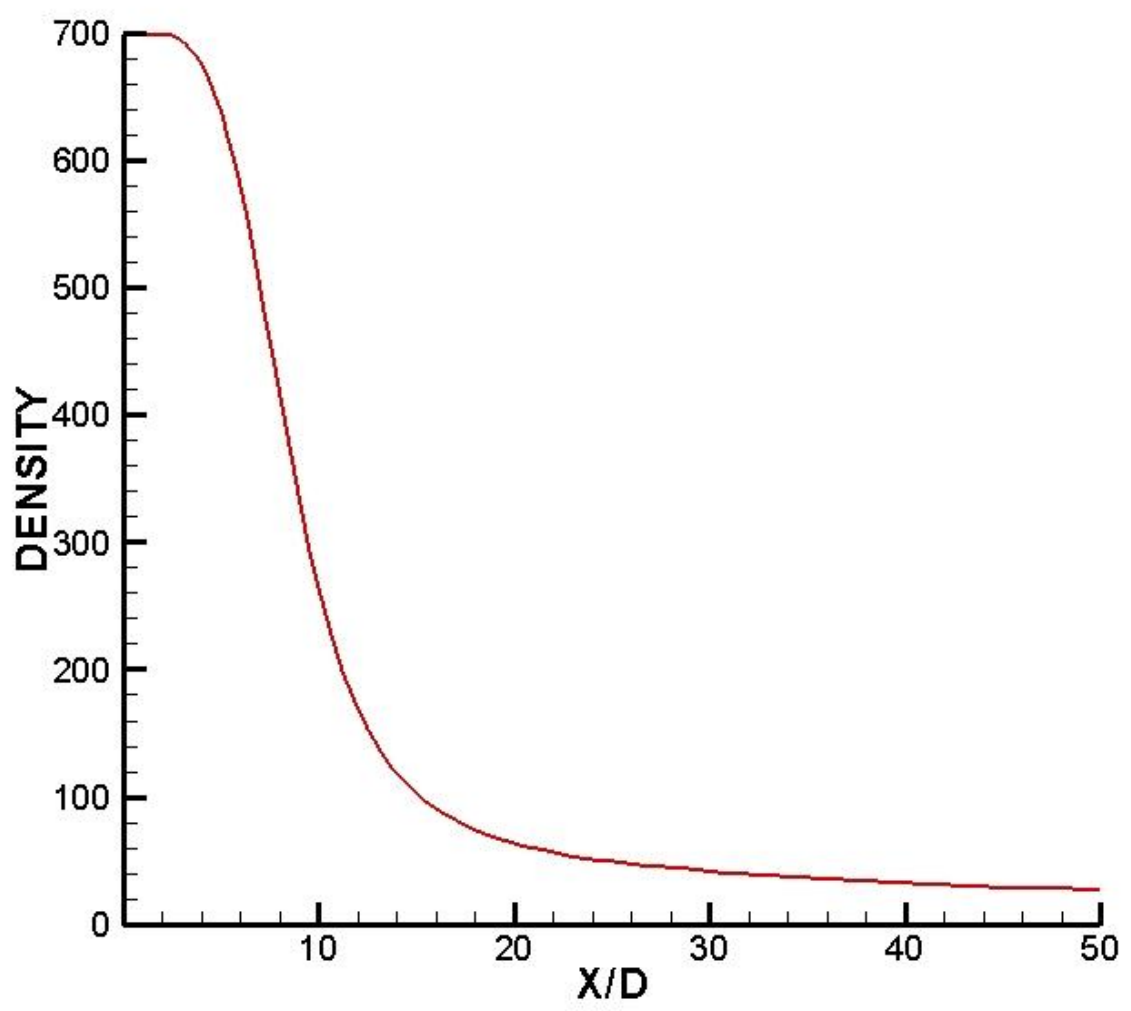

Figure 8 - Axial variation of the centerline density with a density ratio of $\omega=0.035$ and a chamber pressure of $\operatorname{Pr}=0.642$.

The potential core based on the density exhibits its initial value of $\rho=700 \mathrm{~kg} \cdot \mathrm{m}^{-3}$ until an axial distance of $X / D=3$ diameters. Then the centerline density starts to rapidly decrease until an axial distance around $X / D=15$ jet diameters at which the centerline density has a value of approximately $\rho=113 \mathrm{~kg} \cdot \mathrm{m}^{-3}$. After this point the centerline density continues to decrease but at a lower rate and reaching approximately $\rho=27 \mathrm{~kg} \cdot \mathrm{m}^{-3}$ at an axial distance of $X / D=50$ jet diameters. Also like in the case 1 , comparing this graphic with Figure $5-c$ ) it is possible to see that the start of the accentuated decrease of the centerline density is close to the point at which the jet dense core ends. 
The Figure 9 shows the axial variation of the centreline density for the Case 3 described in Table 1.

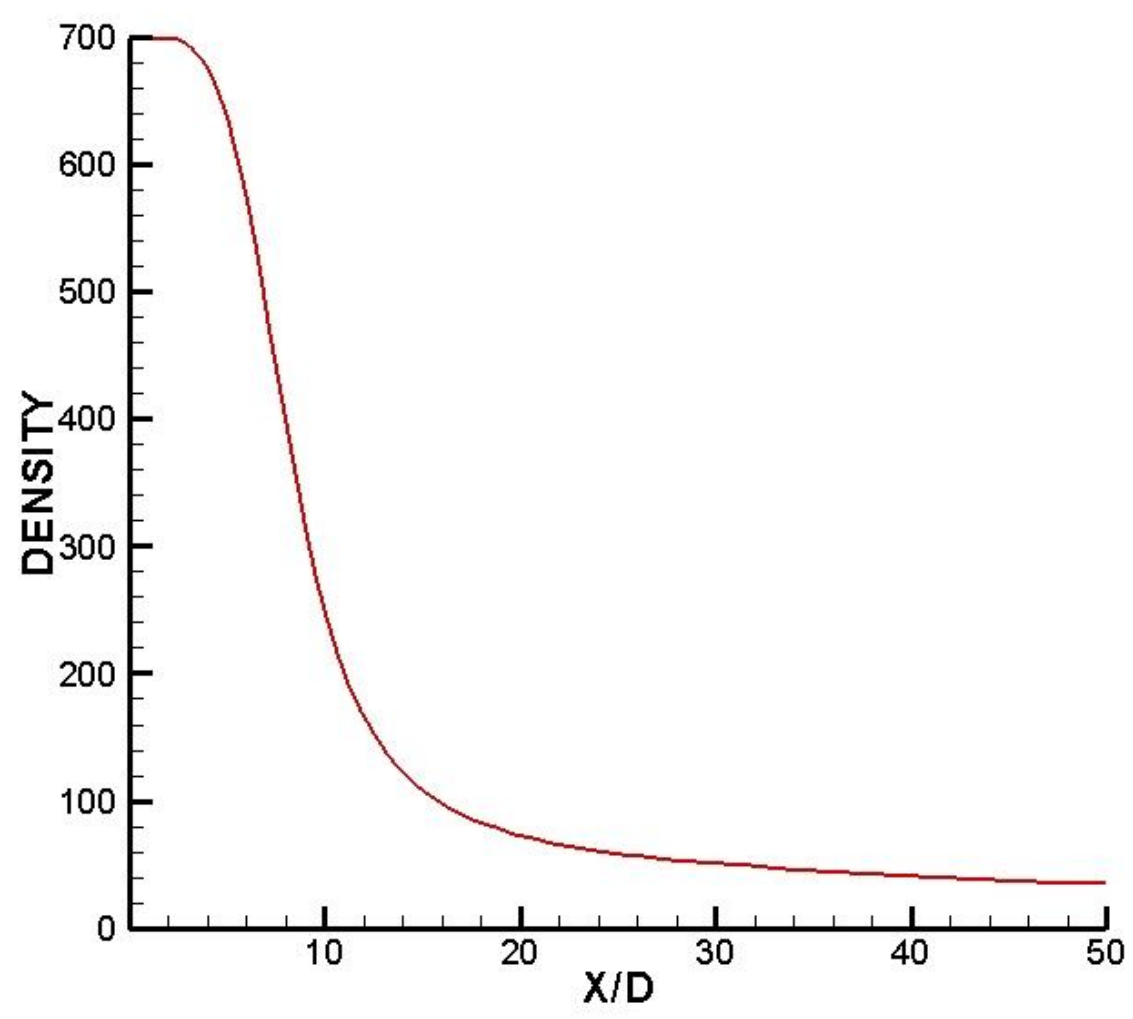

Figure 9 - Axial variation of the centerline density with a density ratio of $\omega=0.045$ and a chamber pressure of $\operatorname{Pr}=0.825$.

Observing the graphic it is concluded that it is very similar to the graphics for cases 1 and 2. The centerline density for this case also remains approximately at its initial value of $\rho=$ $700 \mathrm{~kg} \cdot \mathrm{m}^{-3}$ until approximately an axial distance of $X / D=3$ diameters, again corresponding to the potential core size. Then it undergoes into an accentuated decrease, which corresponds to the jet rapid expansion, until approximately an axial length of $X / D=15$ diameters at which the centerline density has approximately the value of $\rho=108 \mathrm{~kg} \cdot \mathrm{m}^{-3}$. After this, the centerline density continues to decrease, at a lower rate, reaching a value of approximately $\rho$ $=35 \mathrm{~kg} \cdot \mathrm{m}^{-3}$ at $\mathrm{X} / \mathrm{D}=50$ jet diameters of axial distance. Again, comparing this graphic with Figure $6-c$ ) it is possible to see that the start of the accentuated decrease of the centerline density is close to the point at which the jet dense core disappears.

There are no big differences between the axial variation of the centerline density for the three studied cases, with the graphics being very similar for all, and revealing a potential core size of 3 diameters. However, looking to Figure 10, which represent the results shown 
above, in the same graphic and in a logarithmic scale, is possible to notice some small differences and take some conclusions from them. The accentuated decrease of the centerline density starts at the same axial length for all the studied cases. However, in the cases with higher chamber pressure and density ratio the decrease happens at a slightly higher rate, indicating this way a faster expansion despite the pressure gradient, between the injection pressure and the chamber pressure, is lower in these cases. As the ambient fluid density is higher in the cases with higher pressure, after the jet expansion, the density decrease rate slows down faster for the cases with higher chamber pressure and the final jet centerline density is higher for these cases. A final conclusion is that the length and thickness of the dense core slightly decrease as the chamber pressure increases, this conclusion was also achieved by the analysis of Figures $4-c$ ), 5-c) and 6-c) in the last section.

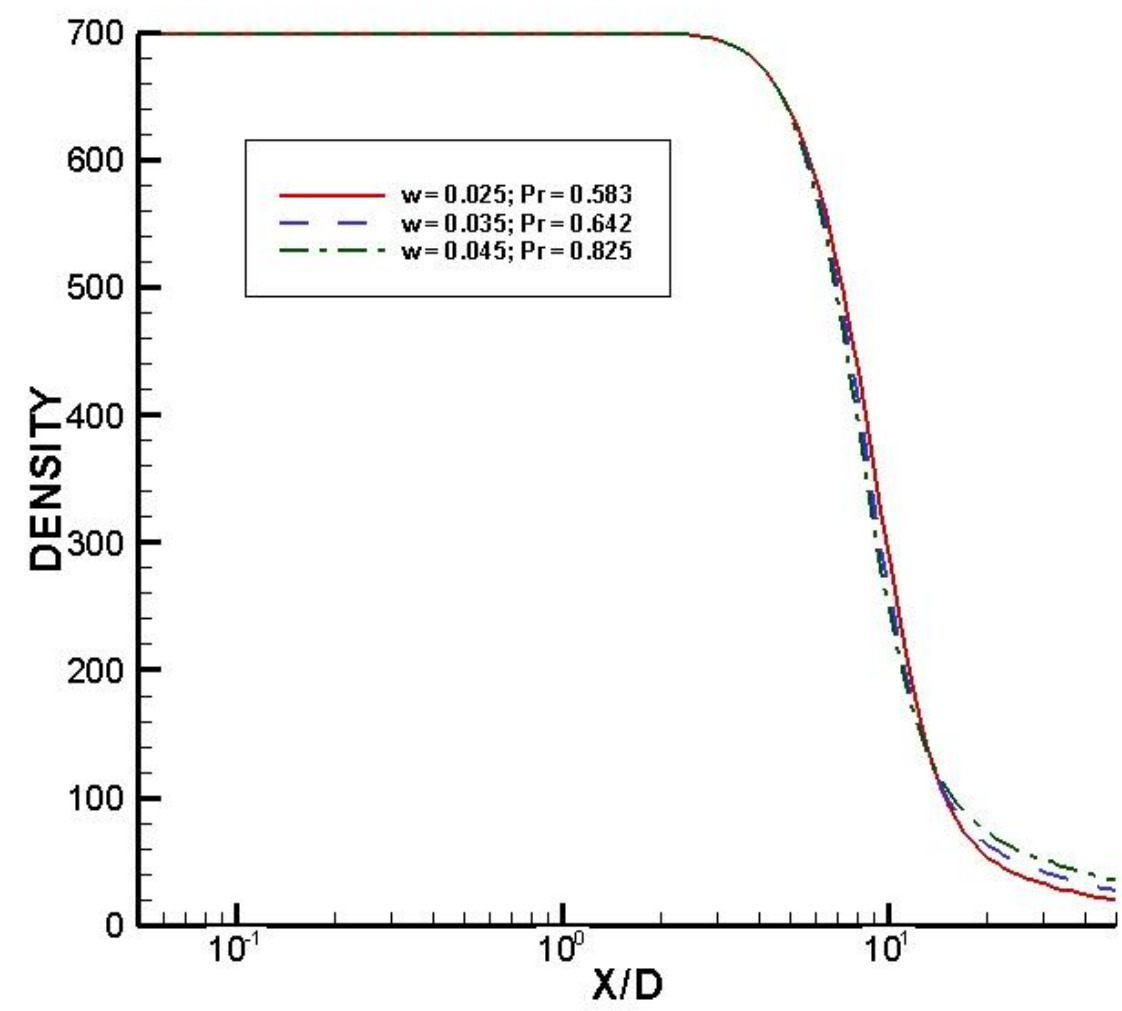

Figure 10 - Axial variation of the centerline density in a logarithmic scale for the cases 1, 2 and 3. 


\subsubsection{Centerline velocity decay for $\omega=0.025,0.035$ and 0.045}

The Figure 11 shows the centerline velocity decay for the case 1 described in Table 1.

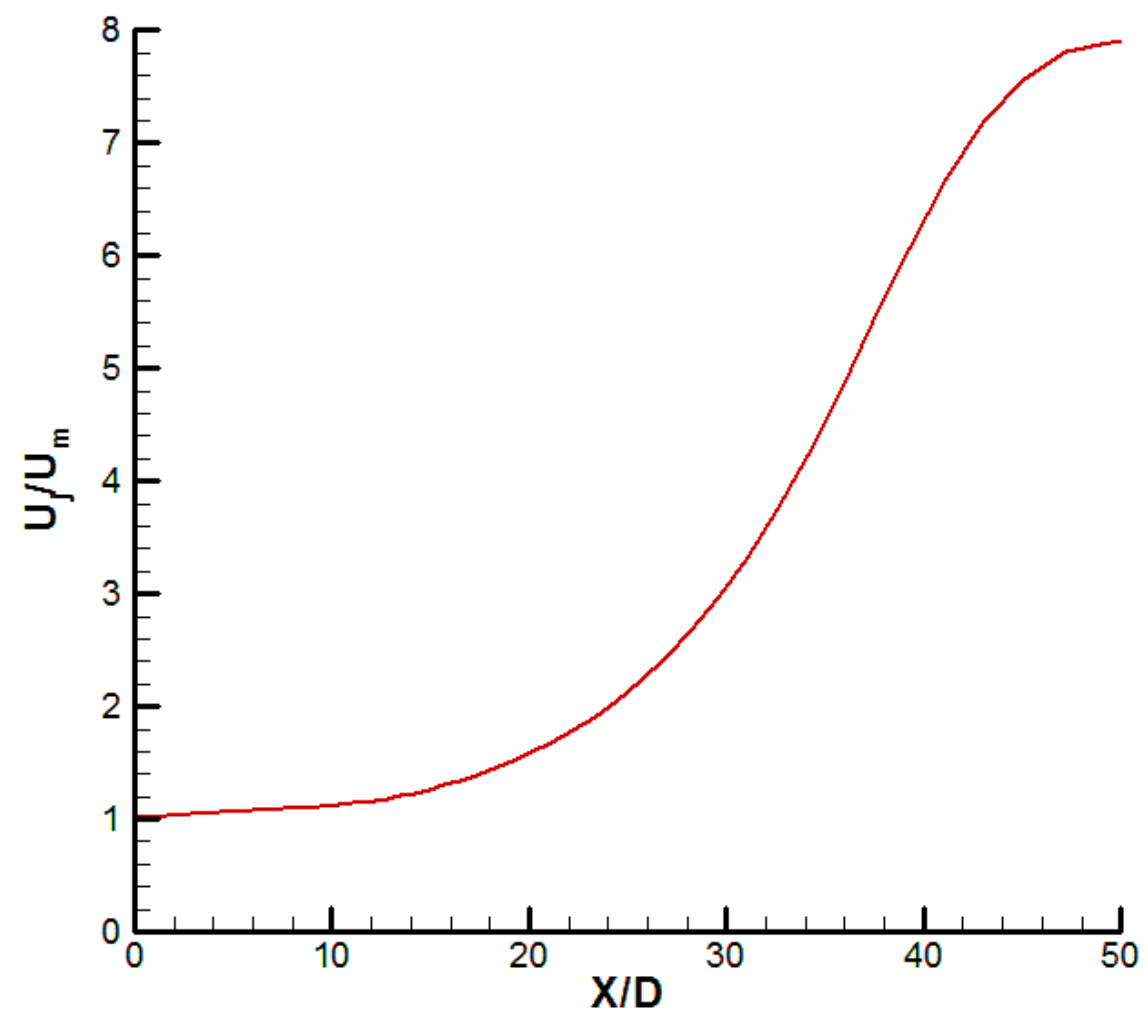

Figure 11 - Centerline velocity decay for a density ratio of $\omega=0.025$ and a chamber pressure of $\operatorname{Pr}=$ 0.583 .

This graphic show, at each point, how many times the centerline velocity has decreased from its initial injection value. Analyzing the graphic it can be seen that the centerline velocity slightly decreases until an axial length of approximately $X / D=15$ injector diameters at which the velocity is 1.27 times lower than the initial velocity. After this axial length the velocity decay rate starts to rise and the velocity experiences a faster decrease, at this stage the velocity decay is almost linear with a rate of $\alpha=0.35280$. Comparing with Figure $4-a$ ), it is possible to see that this increase of the centerline velocity decay correspond approximately to the axial localization of the center of the vortex. This suggests that the vortex can be responsible for the dissipation of a large part of the jet energy. The centerline velocity decreases by half in an axial length of approximately $X / D=24$ injector diameters. The rate of the centerline velocity decay starts to slow down at a length of 45 diameters and at $X / D=50$ diameters the centerline velocity has decayed approximately 7.9 times. 
By the analysis of the present graphic it is possible to determine the potential core size based on the velocity decay. In this investigation it is considered the existence of the potential core until a velocity decay of $10 \%$, thus it was conclude that the potential core based on the centerline velocity decay has a size of $X / D=8.30$ diameters.

The Figure 12 shows the centerline velocity decay for the case 2 described in Table 1.

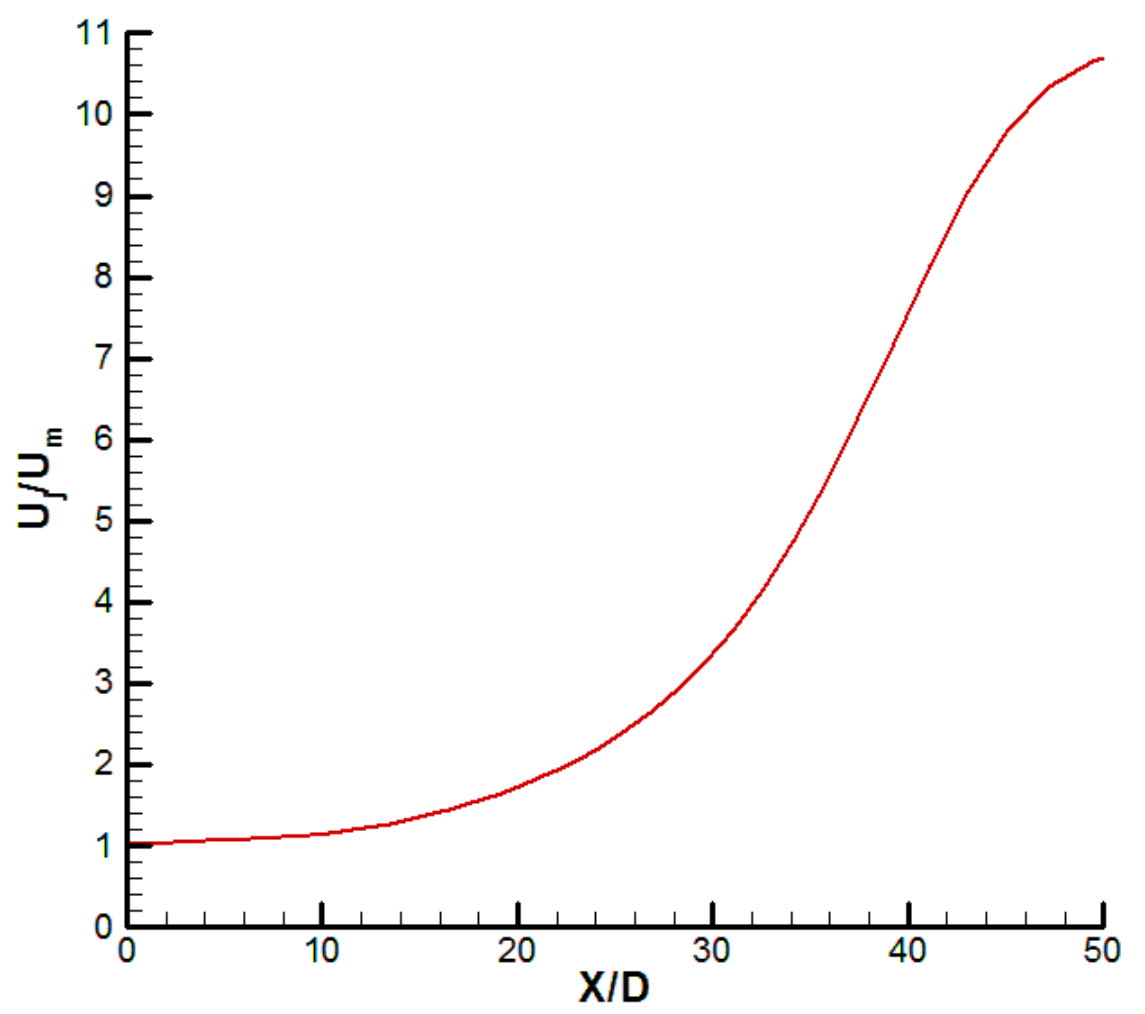

Figure 12 Centerline velocity decay for a density ratio of $\omega=0.035$ and a chamber pressure of $\operatorname{Pr}=$ 0.642 .

By the analysis of the graphic it is observed that, like in the previous case, the centerline velocity decay initially rises at a slow rate and then, after a length of approximately X/D $=15$ diameters, at which the velocity has decayed 1.35 times, the rate of the centerline velocity decay starts to rise. Again the bigger rise of the velocity decay's rate is verified close to the distance at which, in Figure 5-a), is seen the center of the vortex. At this stage, the velocity decay is almost linear with a decay's rate of $a=0.48244$. The centerline velocity has half the value of the initial velocity at an axial distance of approximately $X / D=22.5$ injector diameters. The rate of velocity decay starts to decrease at around X/D $=46$ diameters and at a length of $X / D=50$ diameters the centerline velocity has decayed approximately 10.7 times.

The $10 \%$ decay of the centerline velocity is achieved after an axial length of approximately $\mathrm{X} / \mathrm{D}=7.84$ diameters which corresponds to the potential core size. 
The Figure 13 shows the centerline velocity decay for the case 3 described in Table 1 .

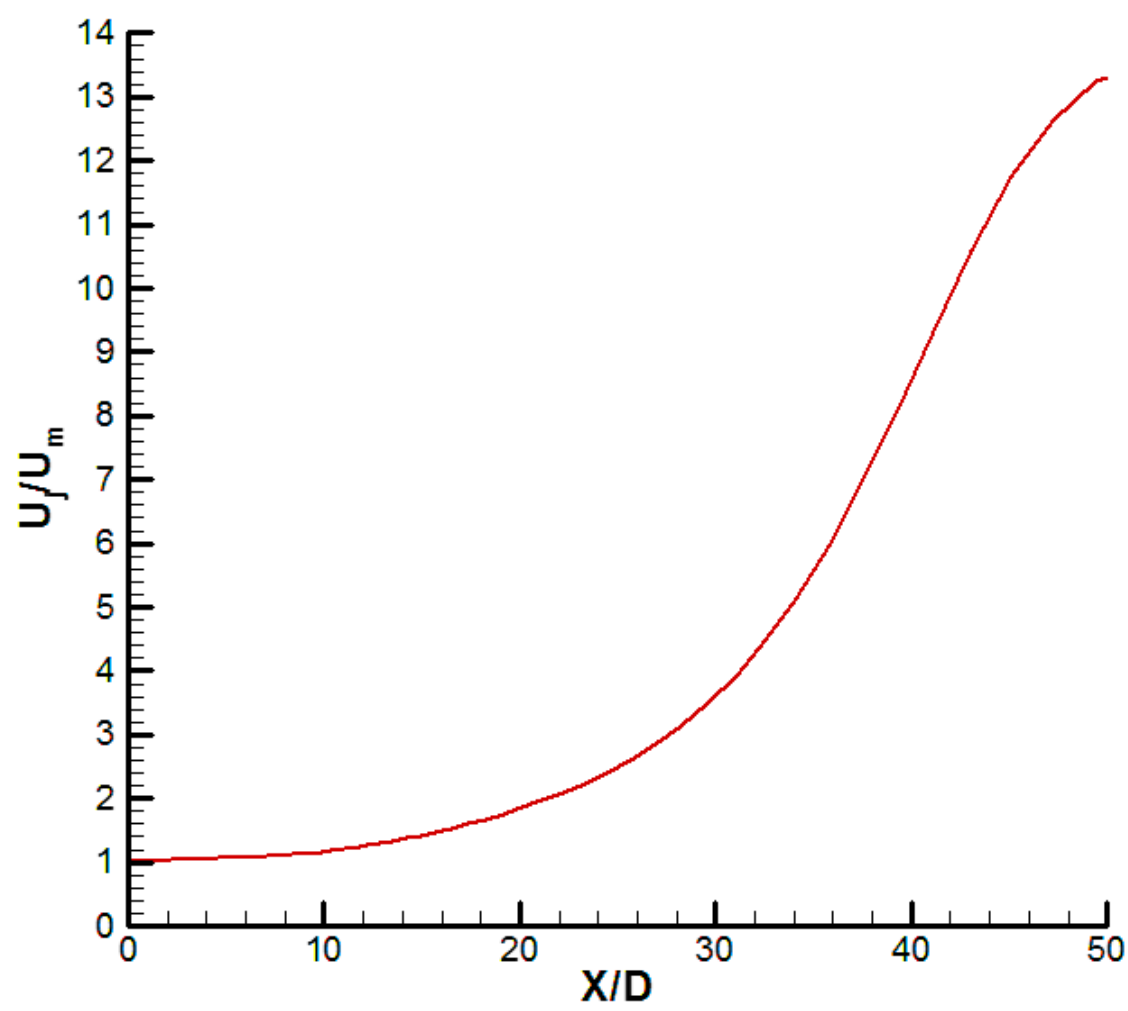

Figure 13 Centerline velocity decay for a density ratio of $\omega=0.045$ and a chamber pressure of $\operatorname{Pr}=$ 0.825 .

Observing the graphic can be seen that the centerline velocity slightly decreases until an axial length of approximately $X / D=15$ injector diameters at which the velocity is 1.43 times lower than the initial velocity. Then, like in previously analyzed cases, the centerline velocity decay rate starts to rise and the velocity experiences a faster decrease, also in this case the greater variation in the centerline velocity decay rate is observed at the same length of the center of the vortex seen on Figure 6-a). The centerline velocity decay has the value equal to 2 , which corresponds to a decrease in the centerline velocity by half, at an axial distance of approximately $X / D=21.6$ diameters. The centerline velocity decay continues to rise at a fast, almost constant, rate of $a=0.62668$, until an axial distance of 48 diameters. Finally at a length of $X / D=50$ diameters the centerline velocity has decayed approximately 13.3 times.

The potential core based on the centerline velocity decay has a length of approximately $\mathrm{X} / \mathrm{D}=7.47$ diameters. 
The kind of evolution of the centerline velocity decay is very similar for the three studied cases, as described above. However at higher density ratios is observed a greater increase of the centerline velocity decay than for lower density ratios. As can be observed in Figure 14, which combines the three results presented above in a single graphic for an easier comparison between each other, the velocity decay is almost equal for the three cases until an axial distance of approximately X/D $=10$ diameters. Then the higher pressure cases starts to have a slightly higher increase which, as the axial distance to the injector increases, becomes a bigger difference. These results complement the conclusions achieved in the join analysis of the Figures 4-a), 5-a) and 6-a) that at higher chamber pressures the jet experiences a major loss of velocity and indicates that the greater loss of velocity happens as the jet passes at the same length of the vortex center, suggesting this way that a great part of the kinetic energy loss by the jet is dissipated by the formed vortex.

One interesting result is the potential core sized based on the centerline velocity decay which is different to the density based potential core size obtained previously. The density based potential core has almost the same size of $X / D=3$ diameters for the three test cases, however, when based in the centerline velocity decay the potential core has a bigger size and is different for the three test cases. It is also observed that the potential core size based on the centerline velocity decay slightly decreases as the density ratio increases. These results are different from the ones obtained for the one-phase flow where usually the potential core based on the centerline density and on the centerline velocity decay have approximately the same value.

The difference between the potential core based on the centerline density and the one based on the centerline velocity decay together with the evidences of the jet expansion, reached in section 3.1.1, suggest that the fluid expansion after injection may contribute with kinetic energy to the flow reducing the centerline velocity decay, and this way increasing the jet potential core based on the centerline velocity decay. 


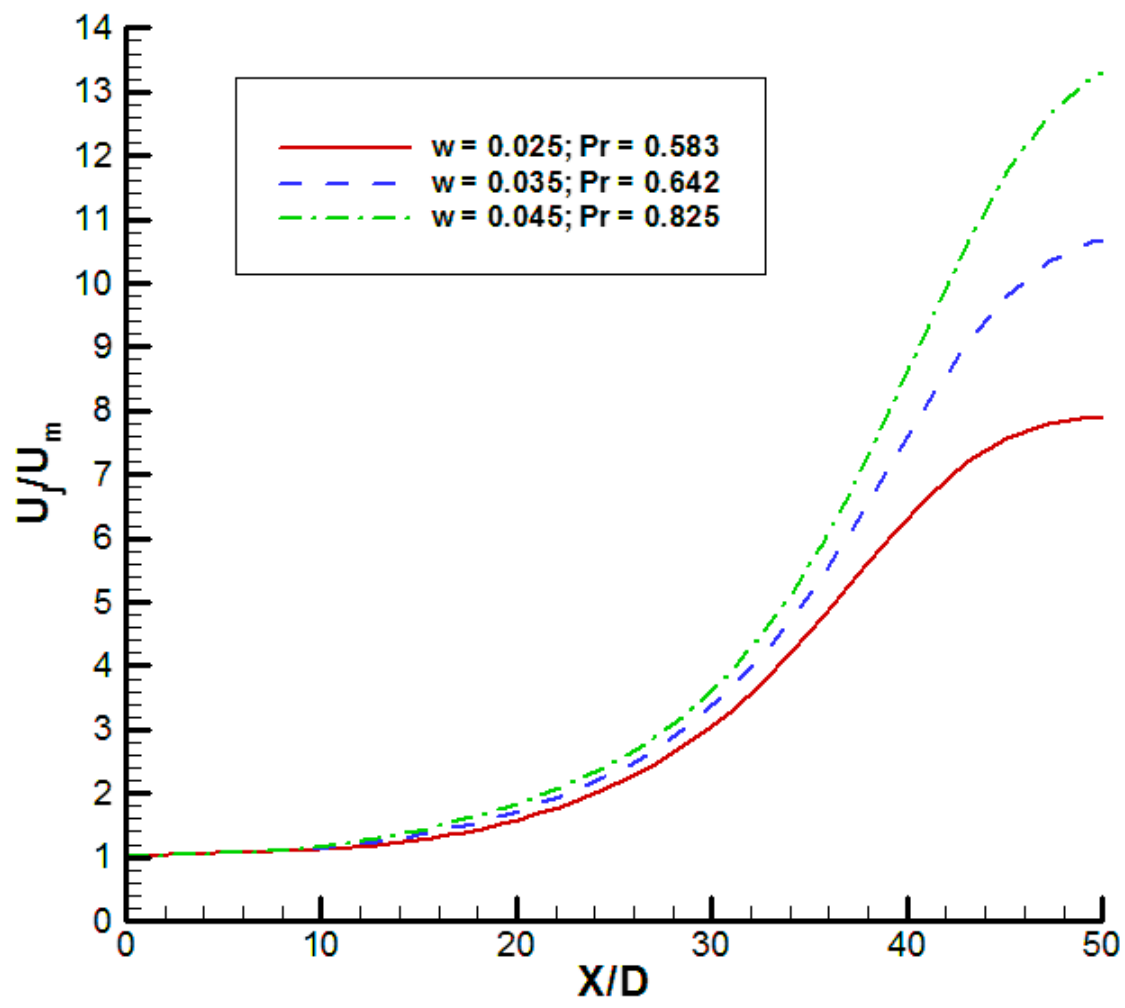

Figure 14 - Centerline velocity decay for the cases 1, 2 and 3 of Table 1. 


\subsubsection{Half width of half maximum of the velocity for $\omega=0.025,0.035$ and}

\subsection{5}

The Figure 15 shows the half width of half maximum of the velocity for the case 1 described in Table 1.

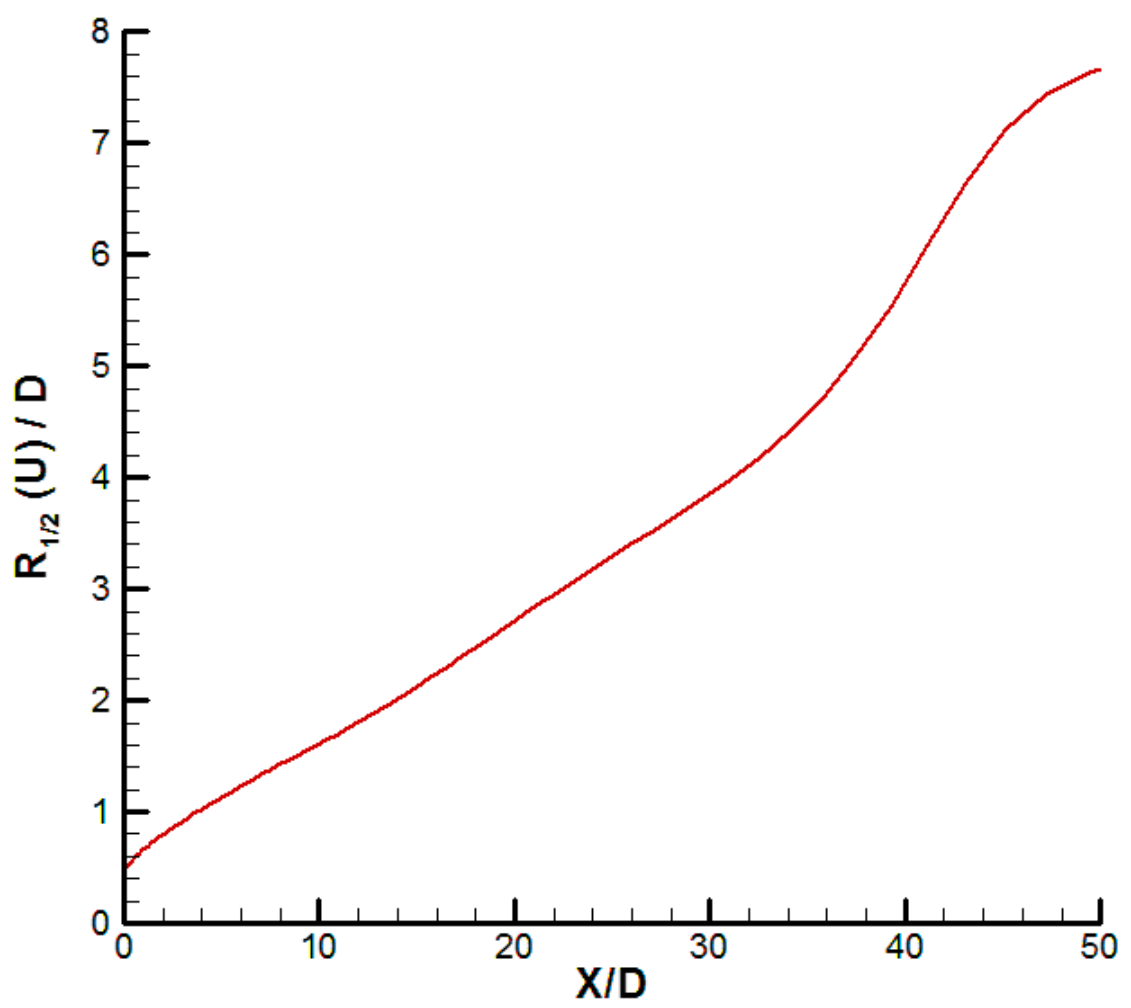

Figure 15 - Half width of half maximum of the velocity for a density ratio of $\omega=0.025$ and a chamber pressure of $\operatorname{Pr}=0.583$.

The radial distance to the centerline normalized by the injector diameter, at which the axial velocity is half of the centerline axial velocity, is represented in this graphic. The radial distance where the axial velocity is half of the maximum is used to determinate the jet growth. Thus, it can be observed in the graphic that the half width of half maximum of the velocity has two different types of progression. One almost linear which lasts from the injector until an axial length of approximately X/D = 34 injector diameters, and other non linear after this length. The linear part of the graphic can be defined by a straight line from which it is possible to determinate the slope. In the non linear part of the graphic, the definition by a straight line is not so easy. For the present work the interest lies in the linear 
section of the graphic and its slope is calculated using two points. The points used were $P_{1}:\left[X / D=10.050, R_{1 / 2}(U) / D=1.6168\right]$ and $P_{2}:\left[X / D=30.062, R_{1 / 2}(U) / D=3.8701\right]$. Thus the slope of the straight line which represents the tangent of the spreading angle of the jet is tan $=0.11261$.

The Figure 16 shows the half width of half maximum of the velocity for the case 2 described in Table 1.

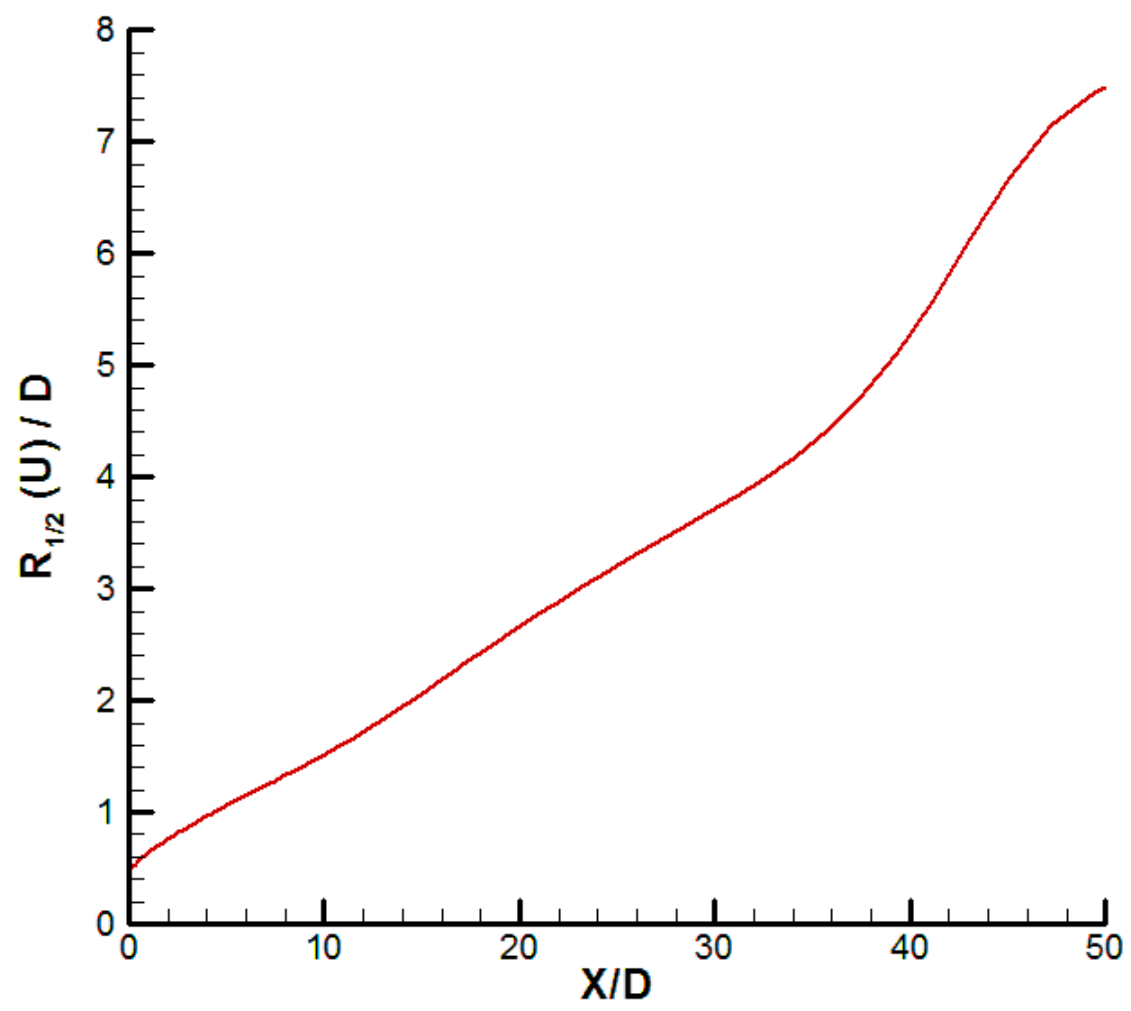

Figure 16 - Half width of half maximum of the velocity for a density ratio of $\omega=0.035$ and a chamber pressure of $\operatorname{Pr}=0.642$.

By the analysis of the graphic it is possible to indentify an almost linear evolution of the half width of half maximum of the velocity that extends since the injector until an axial distance of approximately X/D $=36$ injector diameters and after this point until the end of the graphic a non linear evolution. Choosing two points in the almost linear part of the graphic, $P_{1}:\left[X / D=10.050, R_{1 / 2}(U) / D=1.5206\right]$ and $P_{2}:\left[X / D=30.062, R_{1 / 2}(U) / D=3.7244\right]$, it is possible to draw a straight line with a slope of 0.11016. Again, the straight line's slope represents the tangent of the spreading rate of the jet. The non linear evolution of the half 
width of half maximum of the velocity is made at a bigger rate that the linear part, this is probably caused by the lower velocity in the jet centerline at this part of the domain, this way the half velocity of the centerline is only found at greater radial distances and eventualy, as the jet centerline velocity decays to lower values, becomes impossible to find within the domains of study, a point were the velocity is half of the centerline velocity. After this distance the half width of half maximum of the velocity is no more applicable to study the jet growth.

The Figure 17 shows the half width of half maximum of the velocity for the case 3 described in Table 1.

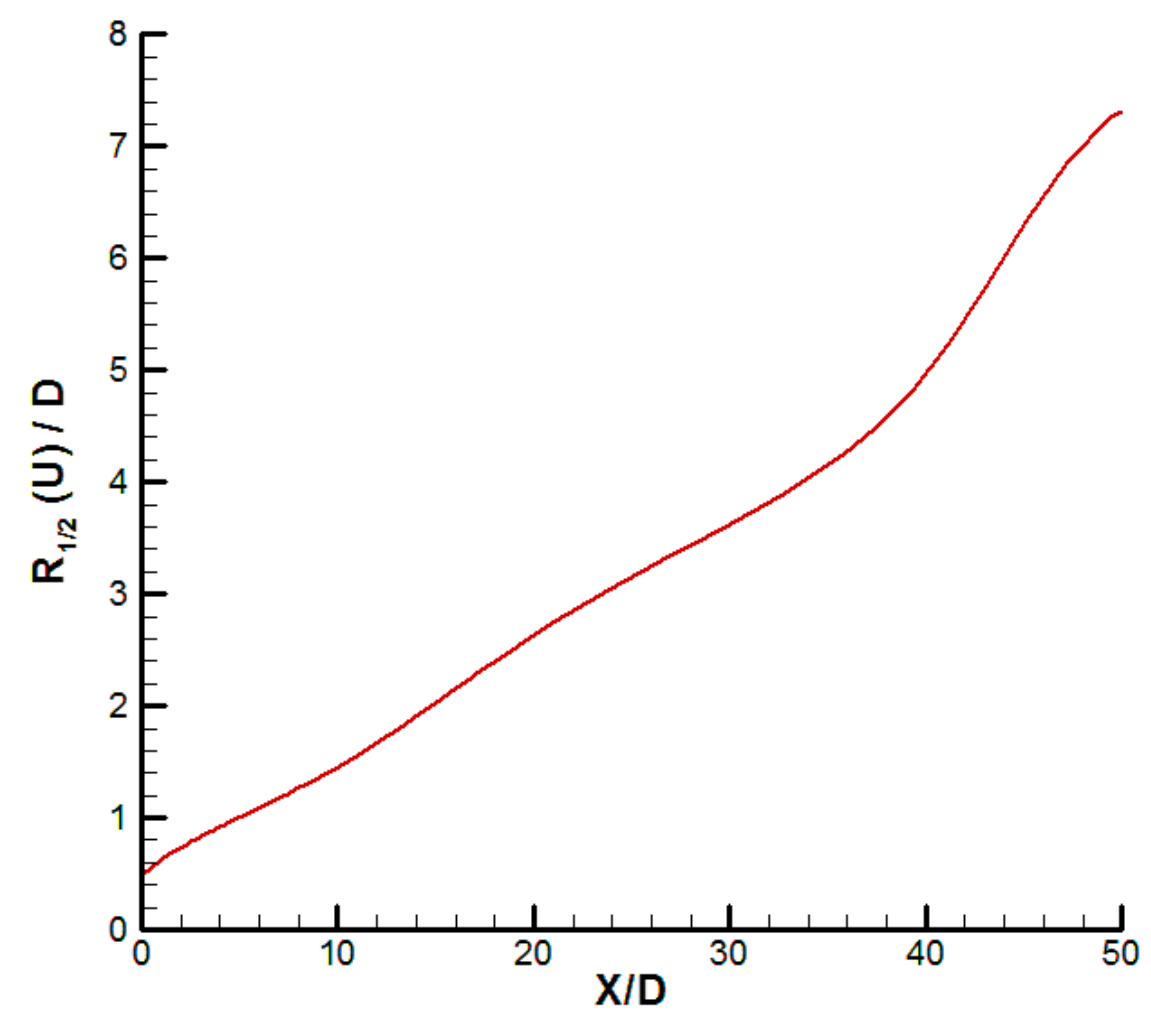

Figure 17 - Half width of half maximum of the velocity for a density ratio of $\omega=0.045$ and a chamber pressure of $\operatorname{Pr}=0.825$.

In this graphic, like in Figures 15 and 16, there are also two different parts, one almost linear part from which is possible to draw a straight line, and one non linear part. The almost linear part of the graphic extends from the injector until approximately a distance of $X / D=$ 37 injector diameters, after this the graphic enter in the non linear part. To draw the straight line that best reflects the half width of half maximum of the velocity in the linear part were 
choosen to different point of the graphic, $P_{1}:\left[X / D=10.050, R_{1 / 2}(U) / D=1.4598\right]$ and $P_{2}:[X / D=$ 30.062, $\left.R_{1 / 2}(U) / D=3.6326\right]$. The calculated slope of the straight line is 0.10857 . As said before, the slope of the straight line represents the jet growth.

Figure 18 includes the results shown above in a single graphic to allow a better comparison between each other. The behavior of the half width of half maximum of the velocity is very similar for the three cases. In all of theme there are one linear and other non linear part. However it is possible to verified that, in the almoast linear zone, the HWHMV slope slightly decreases as the chamber pressure rises indicating that is slightly higher at lower pressures. This result was not expected because in several investigations performed previously it was verified that the spreading rate increased with the increase of the chamber pressure. It can also be verified that for the three cases, the end of the linear zone approximately corresponds, in the graphic of the centerline velocity decay, to the axial distance where the rate of velocity decay is more accentuated. On the other hand, was concluded in the analysis of the centerline velocity decay that the increase of its rate happened after the vortex center and this way could be cause by the vortex existence. Thus, one possible conclusion is that the appearance of the non linear zone of the half width of half maximum of the velocity could be caused by the vortex existence. At the non linear zone the evolution of the HWHMV still remains similar in the three cases but there is an amplification of the HWHMV difference between the cases. Finally it is possible to see that in all cases the HWHMV has a bigger increase in the non linear zone, indicating a bigger spreading rate. The graphic not shows the domain after X/D = 50 diameters, this is because the HWHMV algorithm only functions in a domain were a point with half the centerline velocity can be found. After an axial distance of $\mathrm{X} / \mathrm{D}=50$ diameters the axial velocity is shows a small variation across the radial section and centerline velocity has experienced a high decay (Figures 11, 12, 13 and 14), this way it becomes difficult to find, within the domain, a point with half the velocity of the centerline.

Comparing the half width of half maximum of the velocity graphics with the centerline velocity decay it's possible to observe that, at each case, the jet growth rate has a different value from the centerline velocity decay rate. Also the influence of the chamber pressure variation is different for the two variables. While for the jet growth rate obtained by the HWHMV the pressure change has little effect, with only a slight decrease of the jet growth rate, for the centerline velocity decay rate the increase of the chamber pressure has a greater effect of increasing its value. 


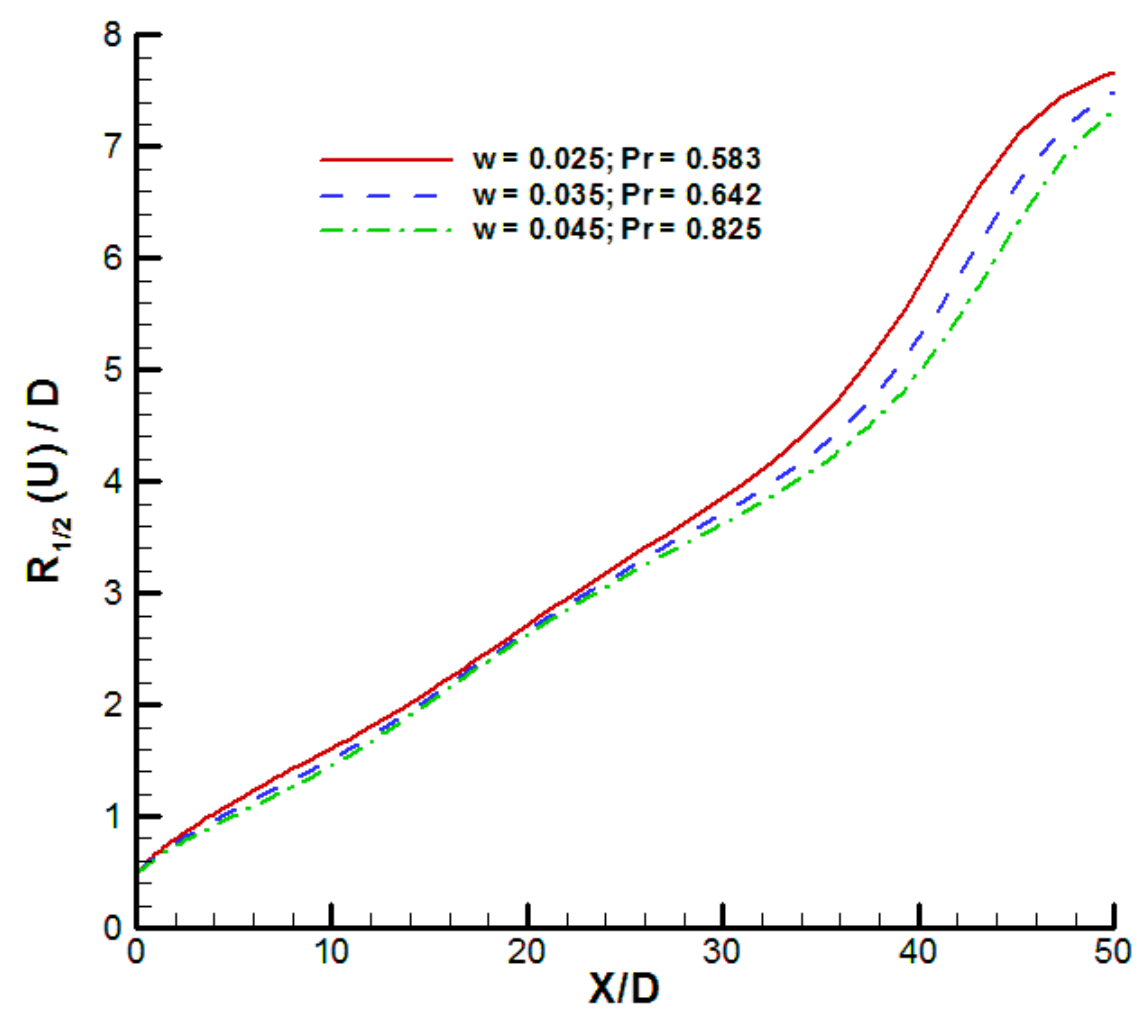

Figure 18 - Half width of half maximum of the velocity for the cases 1,2 and 3 of Table 1.

\subsubsection{Centerline velocity and density decay rate at various chamber-to- injectant density ratios}

Figure 19 shows the decay rates of velocity and density after the potential core for the cases of Table 1 summarizing this way some of the information already shown in figures 7 to 14. The centerline velocity decay rate is calculated using a similar method used for the tangent of the spreading rate. For the centerline density decay were chosen two points in the almost linear part of the graphic of the centerline density variation, the initial centerline density was divided by density at each point and then was made the difference between the two obtained values and divided by the axial distance between the two values. For both velocity and density an increase in the injection chamber pressure has as result an increase of the decay rate which is in agreement with previous works from other authors, however this effect is more pronounced in the velocity decay. The velocity also shows for the three cases a higher decay rate then the density although is important to remember that the density decay starts earlier then the velocity decay due to the longer potential core obtained for the velocity. 


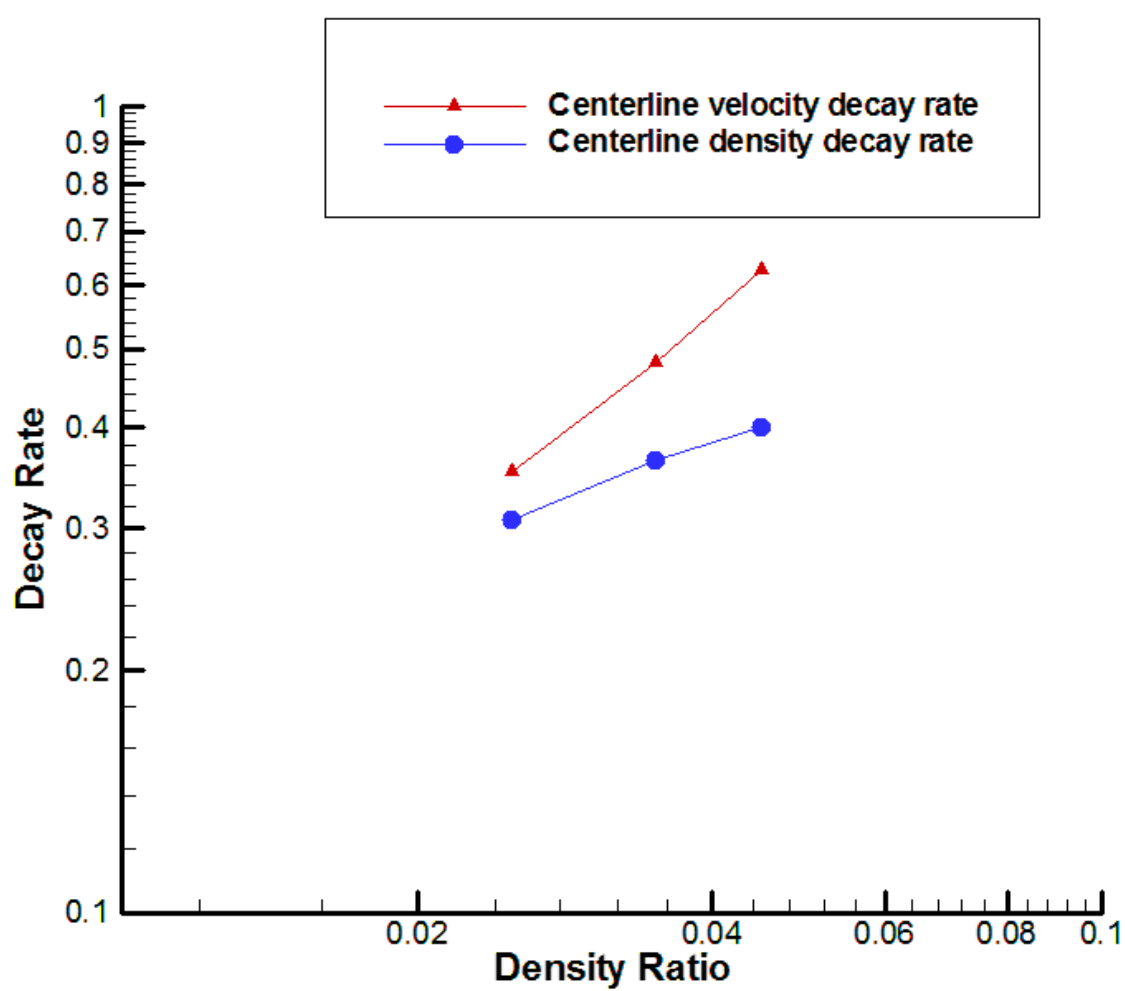

Figure 19 - Decay rate of the centerline velocity and density.

\subsubsection{Tangent of the spreading angle at various chamber-to-injectant density ratios}

Figure 20 shows the tangent of the jet spreading angle calculated by the half width of half maximum of the velocity in order to the density ratio. In addition to the three cases studied previously in the present subchapter the graphic contains three more cases with different density ratios also study in the present work, but for which no detaild results are presented, and results obtained by other authors in previous investigations including the results of Barata et al. [18], the investigation which the present work pretends to extend. The results achieved in the present work are represented in red while those achieved by other authors are represented in black.

The three other cases tested in this work had density ratio values of $\omega=0.010,0.015$ and 0.020 . This cases were not presented in detail at the present work due to the fact that no valid results were found for these cases, as shown in the graphic. The spreading rates for this cases were calculed using the half width of half maximum of the velocity like in the cases presented above but the results obtained are too low in order to be considered valid. The 
analysis of the other results obtained for these cases also shown not to be congruent with any theorys or experimental and numerical results obtained by other authors. Thus these results were simply ignored in the detailed analysis presented previously for the cases 1,2 and 3 of the present work. However, the results obtained for the density ratios of $\omega=0.010,0.015$ and 0.020 were essencial to achieved an important conclusion of the present work. By the analysis of the results obtained for the lower density ratios it is possible to conclude that the present numerical model can not be applied for conditions at which the density ratio between ambient fluid and the injection fluid is bellow approximately $\omega=0.025$.

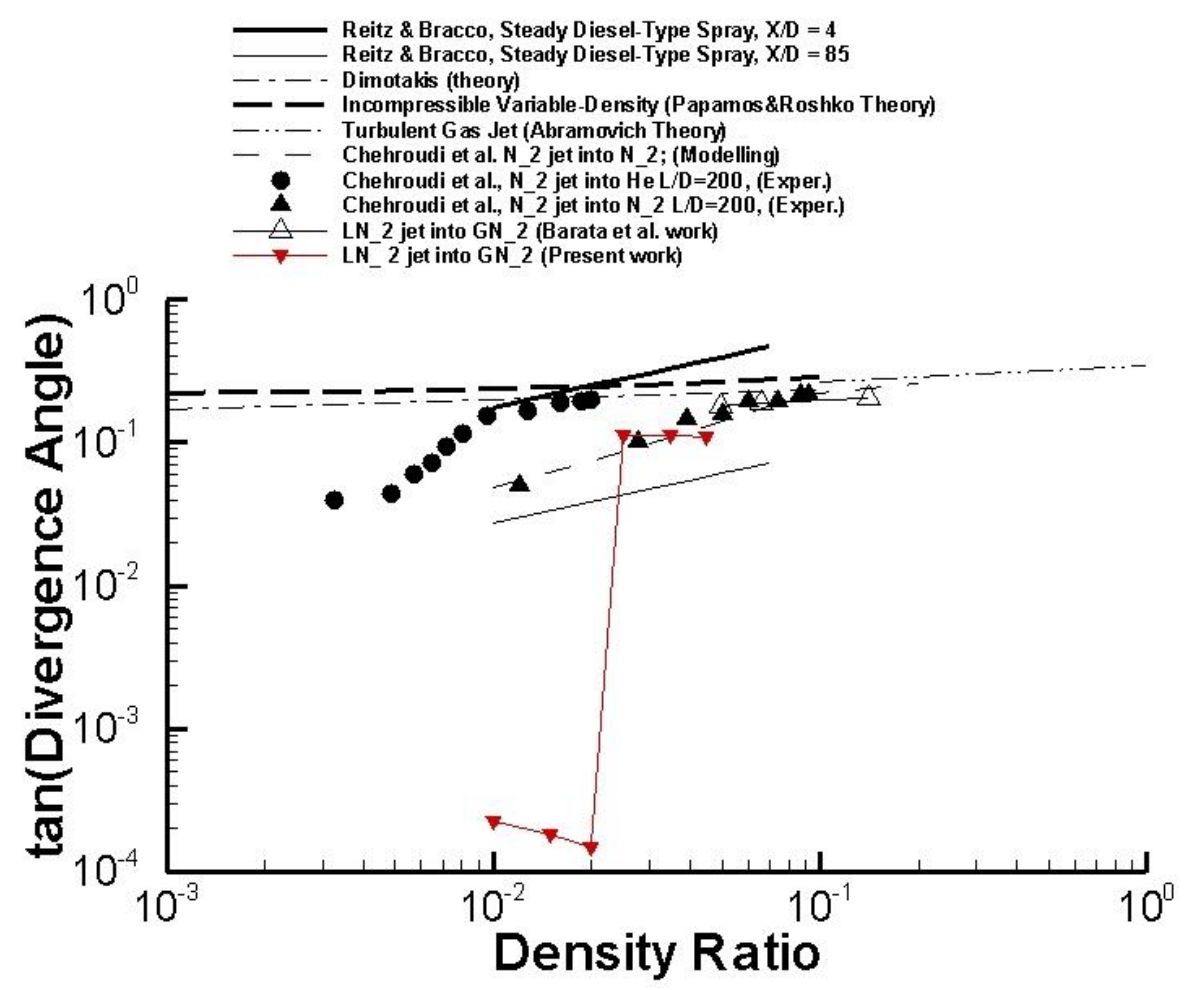

Figure 20 - Tangent of the spreading angle versus the chamber-to-injectant density ratio.

Figure 21 also shows the tangent of the jet spreading angle in order to the density ratio for the cases 1, 2 and 3 studied in the present work and for results of other athors. This graphic is similar to the graphic presented by Barata et al. [18] and to the graphic of Figure 19. But instead of present the results obtained in this investigation for the lower density ratios, like in Figure 20, it presents the limit of applicability of the variable density approach used in the present work. As verified in Figure 20, conditions below a density ratio of $\omega=$ 0.025 can not be predited by the present numerical model, in fact, the numerical model shown not be able to give valid results below this density ratio. Thus, it was established as a limit of applicability of the variable density approach a minimum value of $\omega=0.025$. This limit is represented in Figure 21 by the red vertical line with a black arrow on the rigth. 
The three studied cases are represented in red at the graphic while the results of other author are again represeted in black. It is possible to verified that the results obtain are very close to the experimental results of Chehroudi et al. and also with the modelling of the same authors. However some discrepancies are observed when comparing with results from other authors. While all the other authores have results that show an increase the divergence angle as the density ratio increases, in the present work that does not happens, in fact, the divergence angle shows a sligth decrease as the density ratio increases.

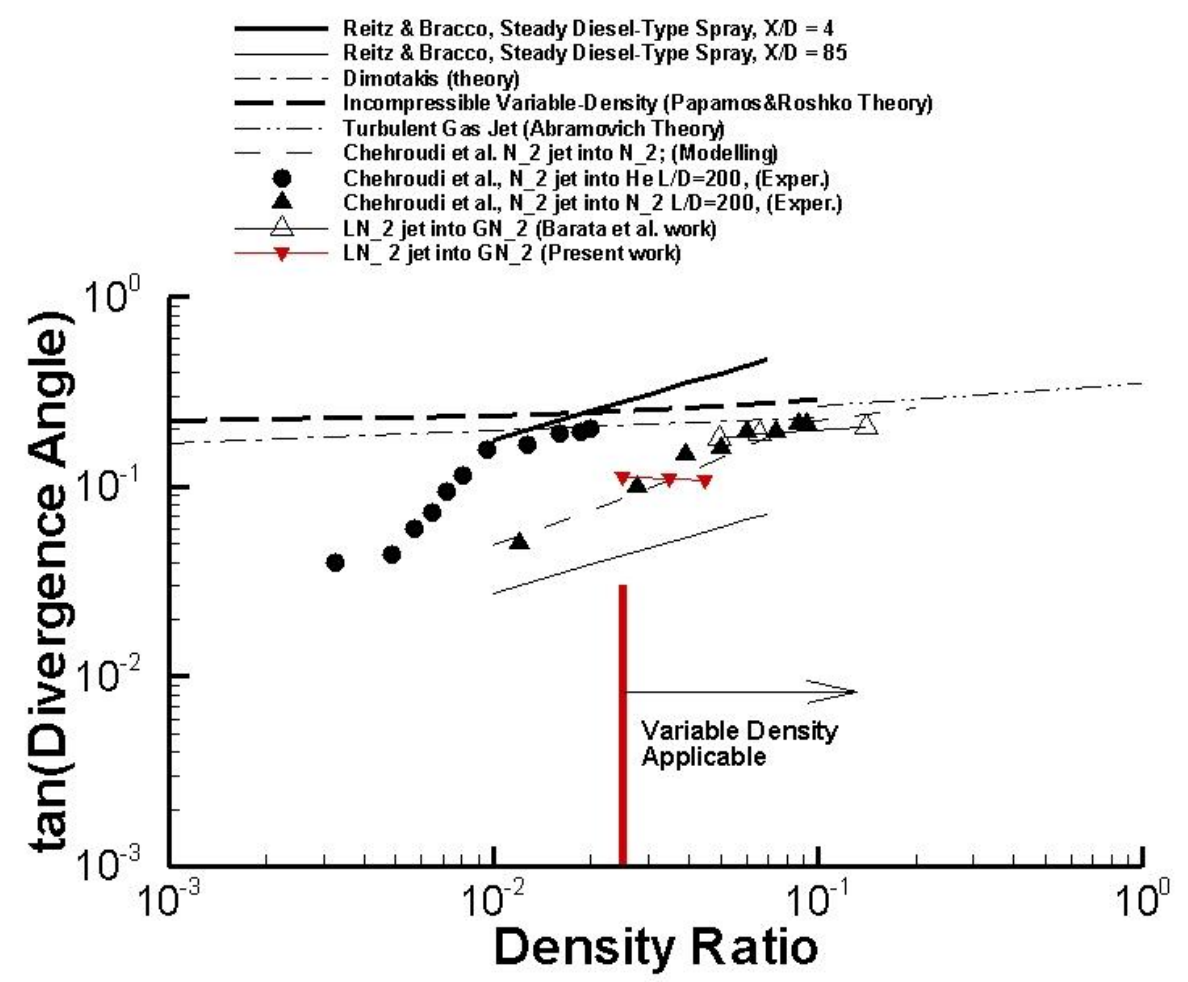

Figure 21 - Tangent of the spreading angle versus the chamber-to-injectant density ratio and representation if limit of applicability of the Variable Density approach. 


\subsection{Study of the injection velocity influence}

The study of the injection velocity $\left(\mathrm{U}_{\text {in }}\right)$ influence in the jet behavior is performed in the present investigation with the main purpose of characterize the mathematical model. This study has a smaller relevance in the present work and therefore it will be not treated as extensively as the density ratio influence study presented in the previous subchapter. The test cases analyzed in this subchapter are presented in Table 2. For this study all the cases have the same chamber-to-injector fluid density ratio of $\omega=0.025$, but the injection velocities are ranging from 3 to $20 \mathrm{~m}_{\mathrm{s}} \mathrm{s}^{-1}$. It is important to refer that the case 4 of Table 2 has exactly the same conditions that the case 1 of Table 1 and so, no more graphics and analysis will be present for this case and it will be only compared with the other cases at the present subchapter. For the other cases will be presented the results obtained by the mathematical model.

In this study no comparisons will be made with theories or results obtained by other authors as the main objective is to identify the behavior change of the mathematical model at different injection velocities.

Figures 22, 23 and 24 show the graphics of the velocity, mixture fraction and density fields for the injection velocities of $U_{i n}=5,10$ and $20 \mathrm{~m} \cdot \mathrm{s}^{-1}$ respectively. In these graphics the horizontal axis represents the axial distance to the injector $(X)$ while the vertical axis represents the radial distance to the centerline of the jet flow (r). In the graphics of the velocity field, the velocity is represented by a vector for each point of the domain where the length of the vector represents the magnitude of the velocity. The mixture fraction and density scalar fields are represented by colored contours and with a color legend to allow the identification of the mixture fraction and density values in the graphic. The mixture fraction is zero when all fluid is chamber fluid and has the value 1 when is injected fluid. These graphics allow the visualization of the main structure of the jet.

Figure 25 shows the axial variation of the centerline density for the four test cases of Table 2. The horizontal axis represents the axial distance to the injector, normalized by the injector diameter, while the vertical axis represents the density expressed in $\mathrm{kg} \cdot \mathrm{m}^{-3}$. These graphics allow the determination of the dense core length and this way helping to know the jet penetration.

Figure 26 shows the centerline velocity decay for the four test cases of Table 2 . The horizontal axis represents the axial distance to the injector, normalized by the injector diameter, while the vertical axis represents axial velocity at the injector divided by the centerline axial velocity. These graphics, like the axial variation of the centerline density, is useful to determinate the jet penetration.

Finally Figure 27 shows the Half Width of Half Maximum Velocity (HWHMV) for the four test cases of Table 2. In these graphics the horizontal axis represents the axial distance to the injector while the vertical axis represents the radial distance to the centerline normalized by the injector diameter. This graphic allows the determination of the jet spreading angle. 


\subsubsection{Velocity and scalar fields for $U_{\text {in }}=5,10$ and $20 \mathrm{~m} . \mathrm{s}^{-1}$}

a)

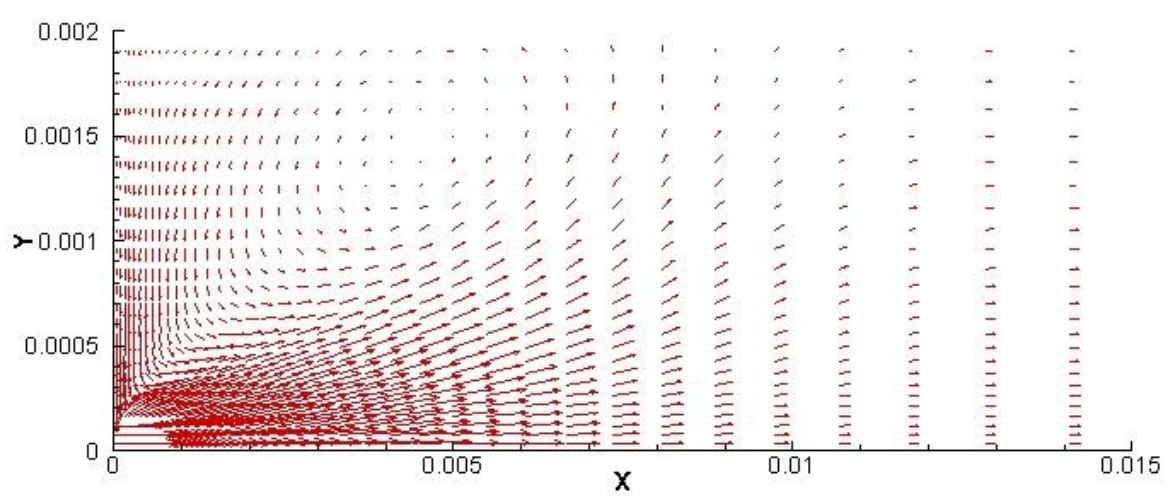

b)

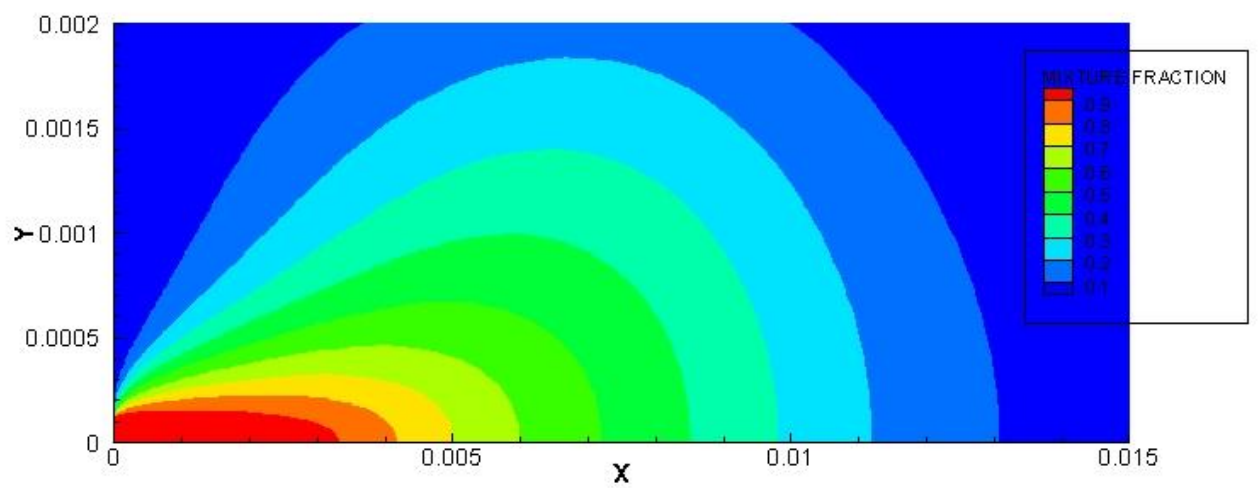

c)

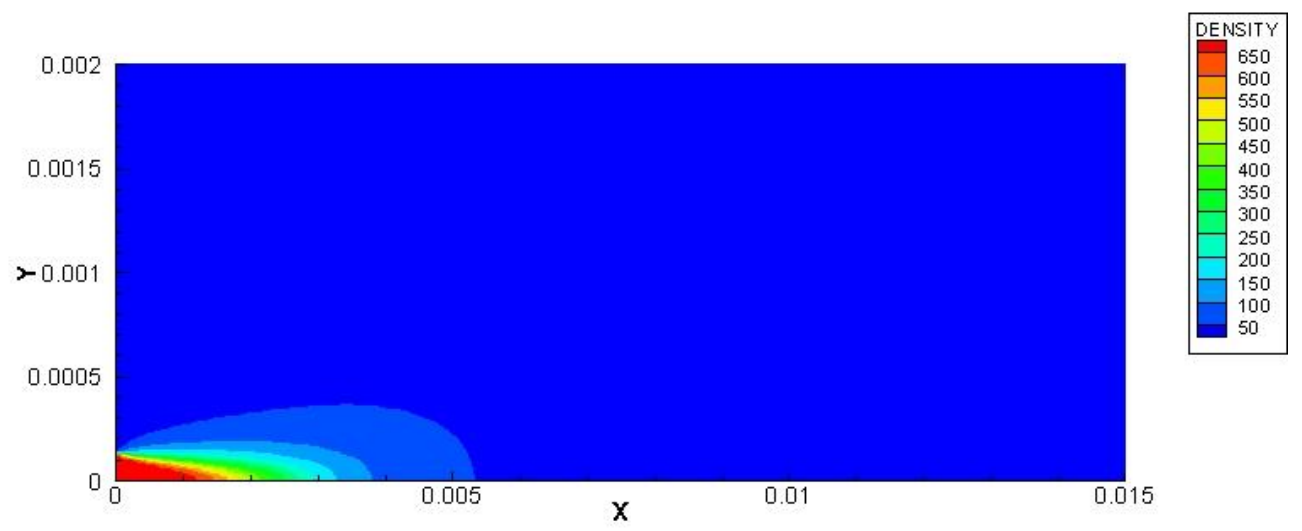

Figure 22 - Velocity and scalar fields of the jet with a density ratio of $\omega=0.025$ and an injection velocity of $U_{\text {in }}=5 \mathrm{~m} \cdot \mathrm{s}^{-1}$, (a) Velocity vectors, (b) Mixture fraction contours, (c) Density contours. 
a)

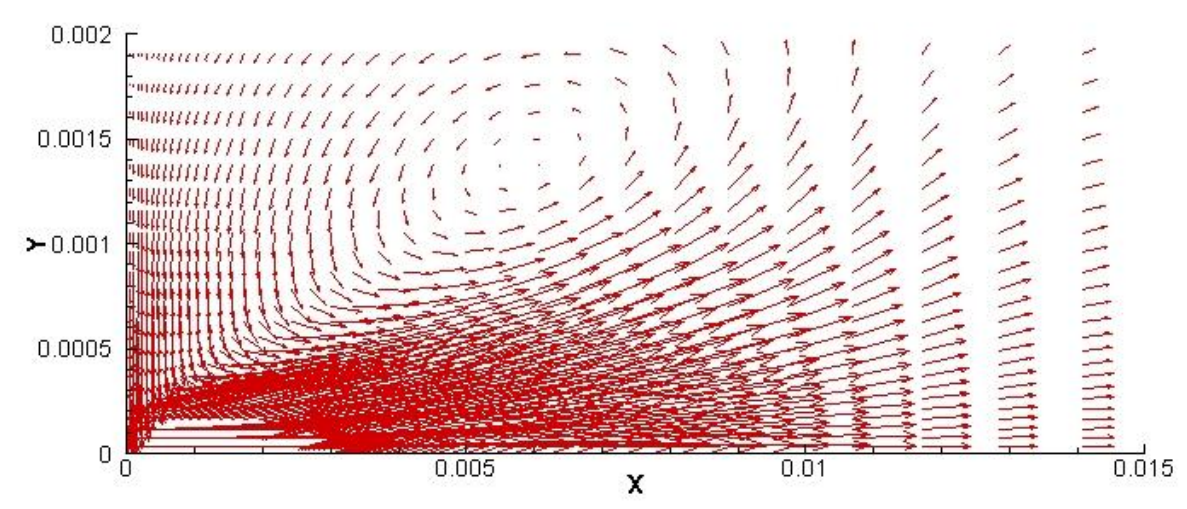

b)

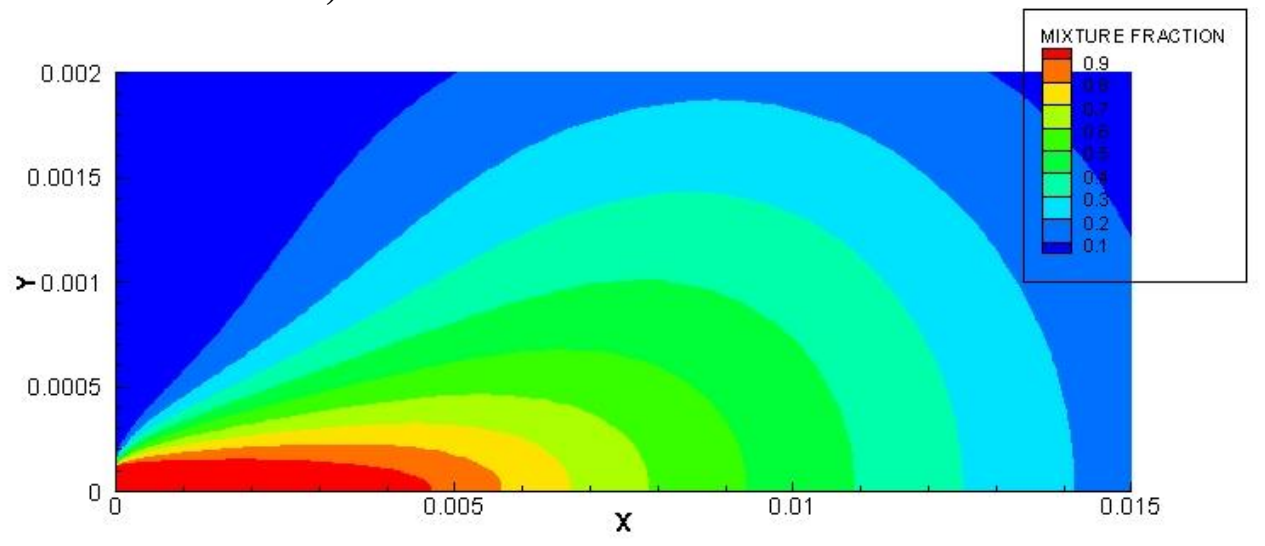

c)

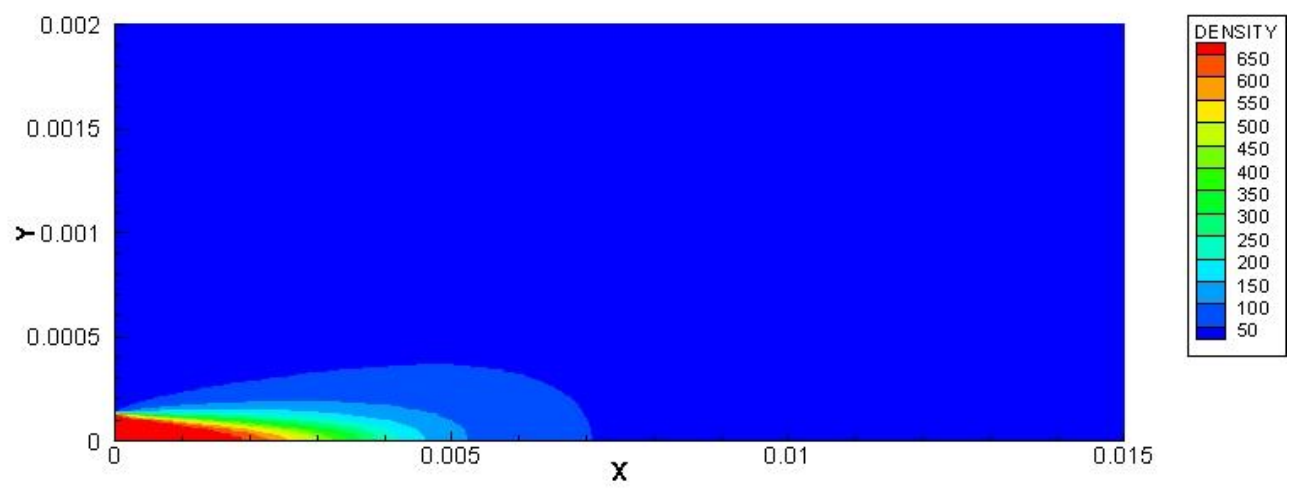

Figure 23 - Velocity and scalar fields of the jet with a density ratio of $\omega=0.025$ and an injection velocity of $U_{\text {in }}=10 \mathrm{~m} \cdot \mathrm{s}^{-1}$, (a) Velocity vectors, (b) Mixture fraction contours, (c) Density contours. 
a)

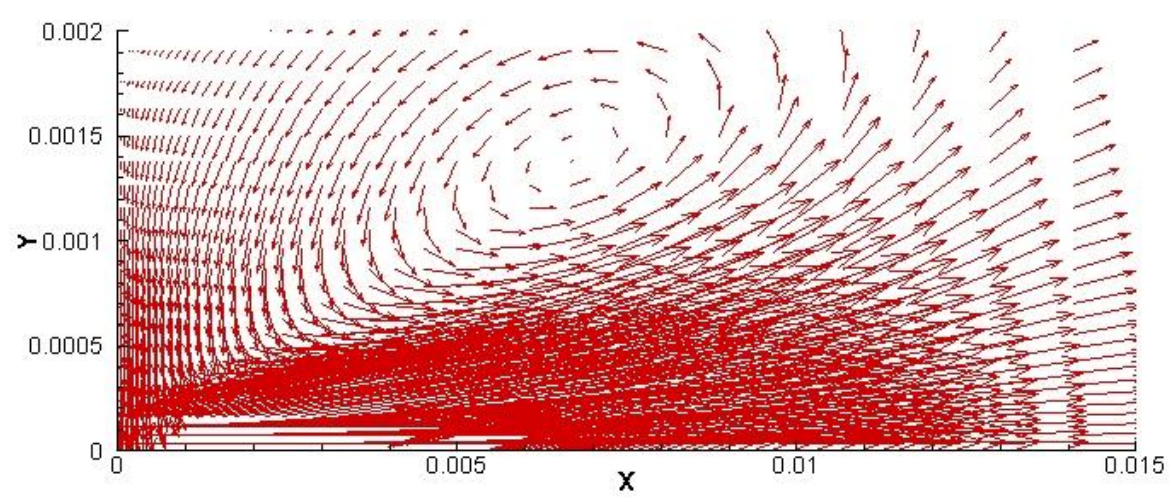

b)

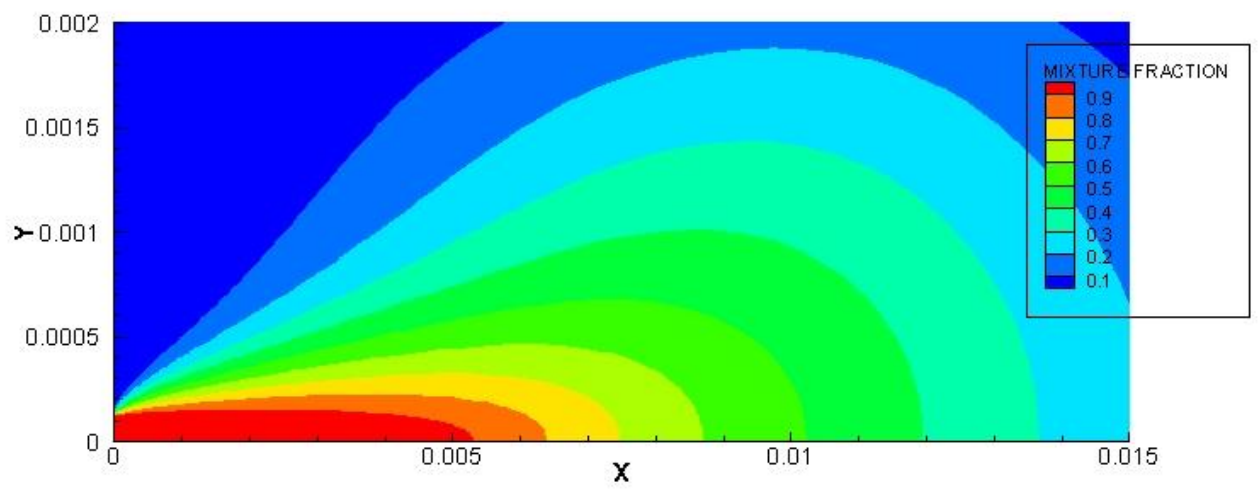

c)

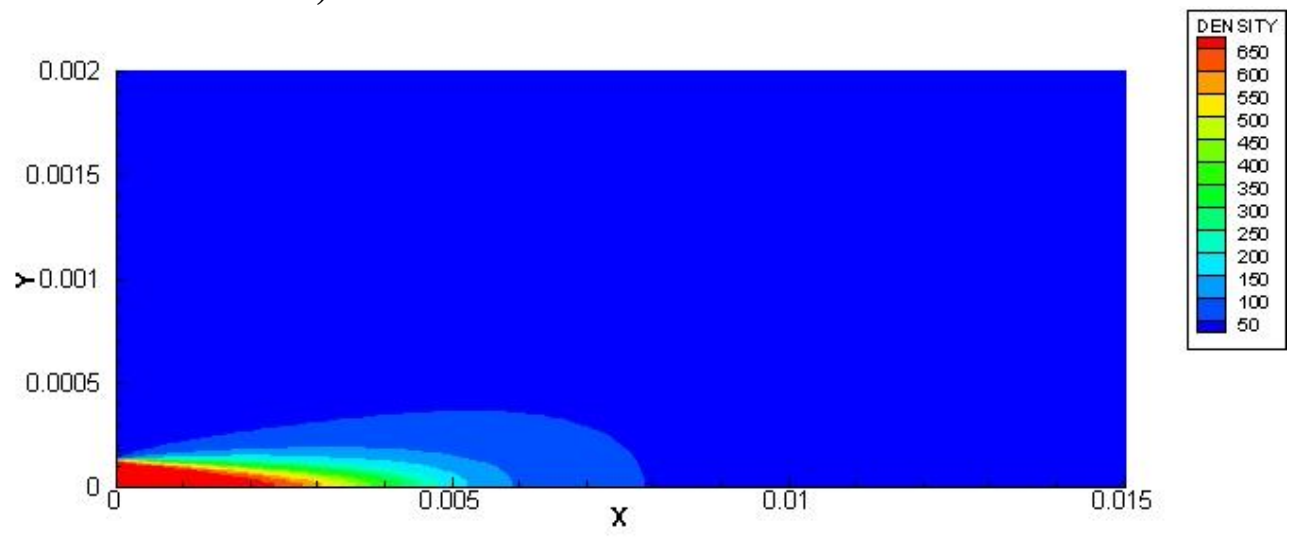

Figure 24 - Velocity and scalar fields of the jet with a density ratio of $\omega=0.025$ and an injection velocity of $U_{\text {in }}=20 \mathrm{~m} \cdot \mathrm{s}^{-1}$, (a) Velocity vectors, (b) Mixture fraction contours, (c) Density contours. 
Figures 22-a), 23-a) and 24-a) show the velocity field for the cases 5, 6 and 7 of Table 2 respectively. Comparing these graphics between each other and with Figure 4-a), case 4, can be concluded that the velocity field for all the injection velocities have the same kind of structure found for an injection velocity of $U_{\text {in }}=3 \mathrm{~m}^{-\mathrm{s}^{-1}}$ (case 4). Figures 22-a), 23-a) and 24a) show the existence of the entrainment phenomena also found in the case 4 . The appearance of a vortex above the jet flow approximately at the same location that in case 4 is visible in Figure 21-a) for the case with a injection velocity of $U_{\text {in }}=5 \mathrm{~m}^{-1} \mathrm{~s}^{-1}$. For the cases 6 and 7 is also observed the appearance of a vortex above the jet flow, however in these cases the vortex has its location further downstream that in cases 4 and 5 , with its center at an axial position of approximately $X=5.8 \mathrm{~mm}$ and $X=6.7 \mathrm{~mm}$ respectively for cases 6 and 7 .

The mixture fraction scalar field is shown in Figures 22-b), 23-b) and 24-b). The case with an injection velocity of $U_{\text {in }}=5 \mathrm{~m} . \mathrm{s}^{-1}$ has almost the same mixture fraction evolution obtained for an injection velocity of $U_{\text {in }}=3 \mathrm{~m} \cdot \mathrm{s}^{-1}$. Increasing the injection velocity to $U_{\text {in }}=10 \mathrm{~m} . \mathrm{s}^{-1}$ the mixture fraction field shows a slower change of the mixture fraction value, thus pointing to a poorer mixing process between the injected and the ambient fluid. A further increase in the injection velocity to $U_{\text {in }}=20 \mathrm{~m} \cdot \mathrm{s}^{-1}$ causes an even poorer mixing process that the one shown in the case with an injection velocity of $U_{\text {in }}=10 \mathrm{~m} \cdot \mathrm{s}^{-1}$, with the mixture fraction at the centerline maintaining a value above 0.9 after an axial length of $X=5 \mathrm{~mm}$.

Figures $22-c$ ), 23-c) and 24-c) show the density scalar field for the cases 5, 6 and 7 of Table 2 respectively. Looking to the Figure $21-c$ ) and comparing it with Figure $4-c$ ) is verified that both are very similar, this way an increment of the injection velocity to $U_{\text {in }}=5 \mathrm{~m} \cdot \mathrm{s}^{-1}$ does not have any visible influence in the jet density scalar field. The same does not happens when the injection velocity increases to $U_{\text {in }}=10 \mathrm{~m} . \mathrm{s}^{-1}$, with this injection velocity the dense core increases and jet expansion becomes slower. A further increase in the injection velocity to $U_{\text {in }}$ $=20 \mathrm{~m} \cdot \mathrm{s}^{-1}$ is responsible for an even greater length of the dense core and a slower jet expansion. These results suggest that despite the increase of the injection velocity $U_{\text {in }}$ the rate of the fluid expansion doesn't increases in the same order and this way the injected fluid shows a smaller interaction with the chamber fluid close to the injector.

The velocity and scalar fields are very similar for the two lower injection velocities. However, as the injection velocity increases for values above $U_{\text {in }}=10 \mathrm{~m} . \mathrm{s}^{-1}$ the velocity and scalar field experienced some changes. So, the injection velocity must be considered as a variable which affects the result obtained for the velocity and scalar field of density and mixture fraction in the application of the mathematical model used in the present work. 


\subsubsection{Axial variation of the centerline velocity for $U_{\text {in }}=3,5,10$ and 20}

$\mathrm{m} \cdot \mathrm{s}^{-1}$

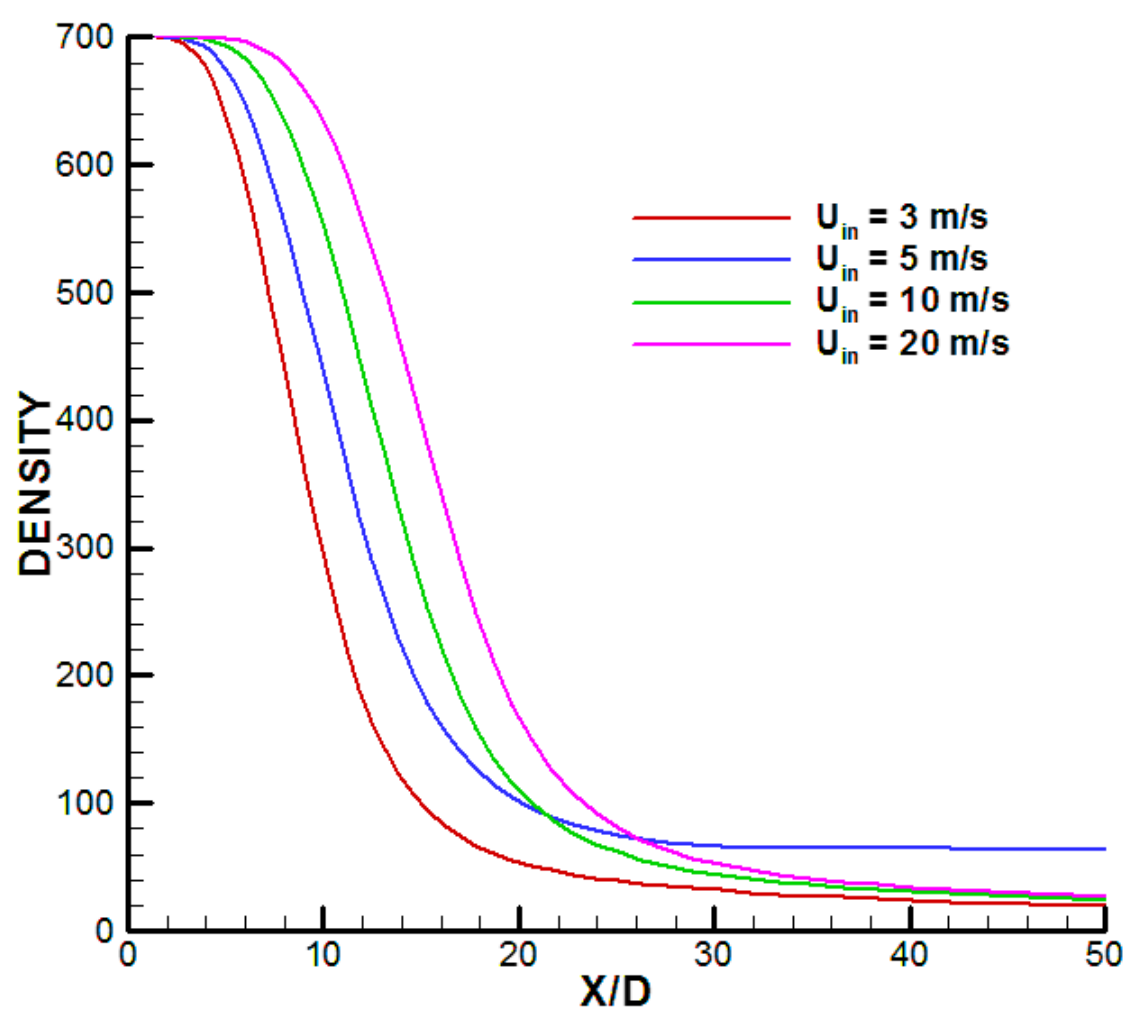

Figure 25 - Axial variation of the centerline density for the cases 4, 5, 6 and 7 of the Table 2 .

The Figure 25 shows the axial variation of centerline density for the studied cases. Having the case 4 as reference, by the observation of the graphic it is possible to see that an initial increase of the injection velocity to $U_{\text {in }}=5 \mathrm{~m}^{-1} \mathrm{~s}^{-1}$ causes the centerline density to decrease at a slower rate. The case 5 also shows a longer potential core based on the density with a length of $X / D=6.36$ diameters when compared with the $X / D=5.16$ diameters found in case 4 , for this study the potential core based on the density is calculated by the axial distance where the density has decayed $10 \%$. At an axial length of around 15 diameters the density decrease rate starts to slow down and at a length of approximately $X / D=30$ diameters the centerline density becomes almost constant until a length of $X / D=50$ injector diameters were the density has a value around $60 \mathrm{~kg} \cdot \mathrm{m}^{-3}$, much higher than in the other cases. In case 6 the centerline density starts to have a lower decrease rate than the case 5 , however at a length of 15 diameters continues to decrease at a higher rate that the previous case and after an axial length of around X/D $=21$ diameters the centerline density for an injection velocity of $U_{\text {in }}=10 \mathrm{~m} \cdot \mathrm{s}^{-1}$ is lower that for $U_{\text {in }}=5 \mathrm{~m} \cdot \mathrm{s}^{-1}$. For the case 6 the potential core shows $\mathrm{a}$ 
length of $X / D=8.11$ diameters. Finally the test case 7 , with an injection velocity of $U_{i n}=20$ $\mathrm{m} . \mathrm{s}^{-1}$, the centerline density shows a lower decrease rate than in the previous case following a similar difference from case 6 that this case had to the case 5 . It is also observable in this case a bigger potential core than in the previous cases with a length of approximately $X / D=$ 10.0 diameters. At an axial length of $X / D=50$ diameters the value centerline density for case 7 reaches approximately the same value observed in cases 4 and 6 .

Here is seen that the centerline density evolution can be highly affected by the injector velocity, having the tendency to follows a slower evolution with the increase of the injection velocity, so, it must be taken in consideration when the objective is to study the density variation.

\subsubsection{Centerline velocity decay for $U_{\text {in }}=3,5,10$ and $20 \mathrm{~m} . \mathrm{s}^{-1}$}

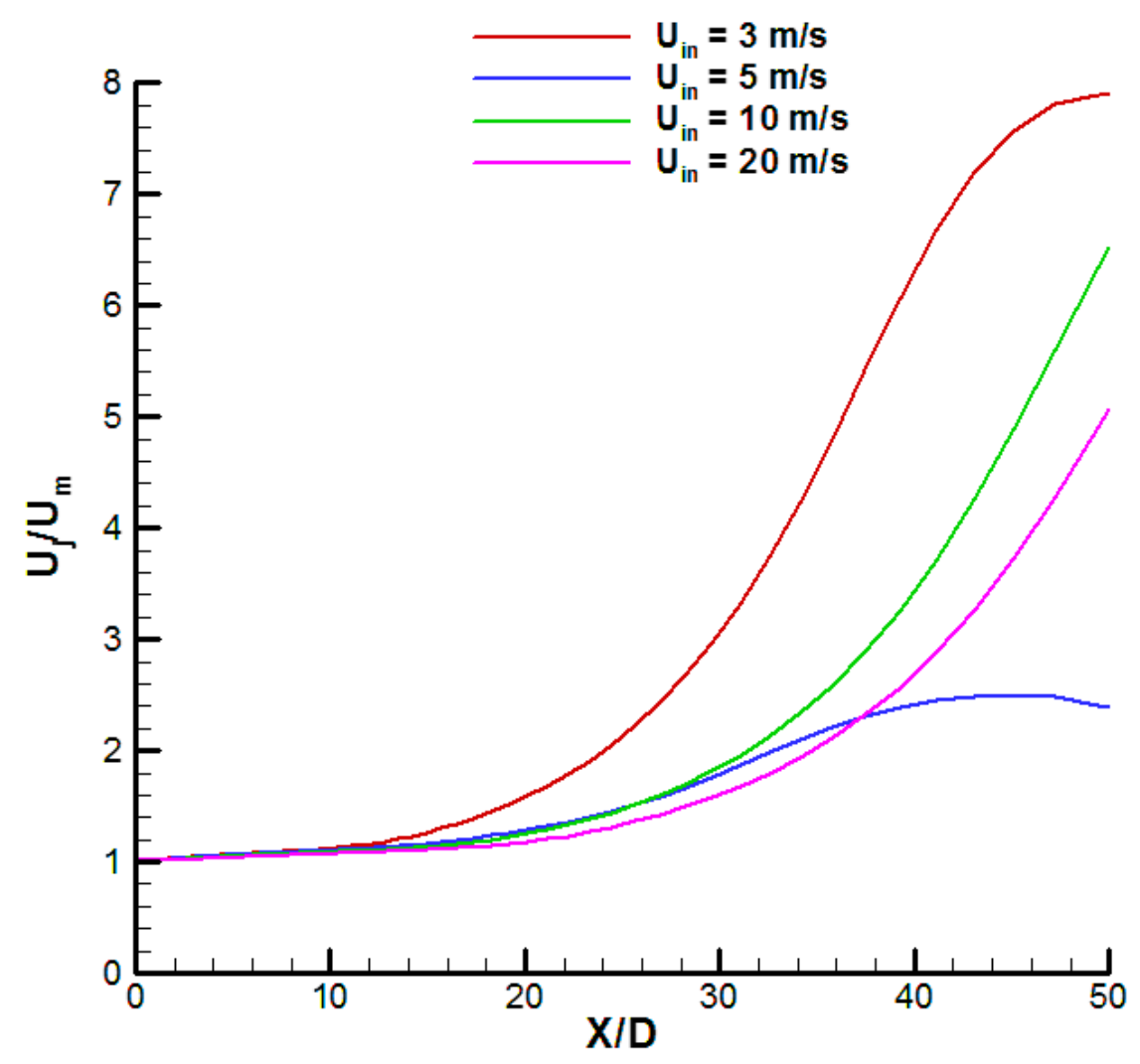

Figure 26 - Centerline velocity decay for the cases 4, 5, 6 and 7 of the Table 2.

The Figure 26 shows the centerline velocity decay for the studied cases. It is observed that the case with higher velocity decay is the one with lower injection velocity, $U_{\text {in }}=3 \mathrm{~m} \cdot \mathrm{s}^{-1}$. A 
small increase on the injection velocity to $U_{\text {in }}=5 \mathrm{~m} \cdot \mathrm{s}^{-1}$ causes the centerline velocity decay at a length of $X / D=50$ diameters to decrease from around 7.9 to 2.4 , which represents a decrease of approximately 70\%. A further increase in the injection velocity to $U_{\text {in }}=10 \mathrm{~m} . \mathrm{s}^{-1}$ is responsible for an increase in $170 \%$ of the centerline velocity decay comparatively to the case 5 , reaching a value of 6.5 at an axial length of $X / D=50$ injector diameters. In the case with higher injection velocity, $U_{\text {in }}=20 \mathrm{~m} \cdot \mathrm{s}^{-1}$, the centerline velocity decay at an axial length of $X / D=50$ injector diameters reaches a value of approximately 5.1 which, compared with the previous case corresponds to a decrease of approximately $22 \%$.

By the analysis of the graphic it is possible to calculate the potential core based on the velocity decay as well as the rate of the centerline velocity decay in the almost linear part for the four studied cases. Case 5 shows a bigger potential core with a length of $X / D=9.40$ diameters when compared with case 4 which has a potential core of $X / D=8.30$ diameters. This trend of an increase of the potential core with the increase of the injection velocity happen in both other cases is also verified cases 6 and 7 with potential core lengths of respectively $X / D=11.5$ diameters and $X / D=14.02$ diameters. On the other hand the rate of the centerline velocity decay in the almost linear part follows the opposite trend. The case 4 has a rate of velocity decay of approximately $\alpha=0.353$, in case 5 it is not possible to identify an almost linear part of the graphic, using the same point used in case 4 is possible to achieved a rate of $a=0.0715$ which is a value quite different from the values achieved for other injections velocities, finally cases 6 and 7 show a rate of velocity decay of respectively $a=0.319$ and $a=0.251$.

Was demonstrated by the present graphic that the injection velocity has influence in the centerline velocity decay. The general trend observed is that an increase of the injection velocity causes a decrease of the centerline velocity decay, however it is observed that the case 5 with an injection velocity of $U_{\text {in }}=5 \mathrm{~m} \cdot \mathrm{s}^{-1}$ does not fallows this trend. Thus, the injection velocity influence must be taken into account when studying the centerline velocity decay. 


\subsubsection{Half width of half maximum of the velocity for $U_{\text {in }}=3,5,10$ and 20 $\mathrm{m} \cdot \mathrm{s}^{-1}$}

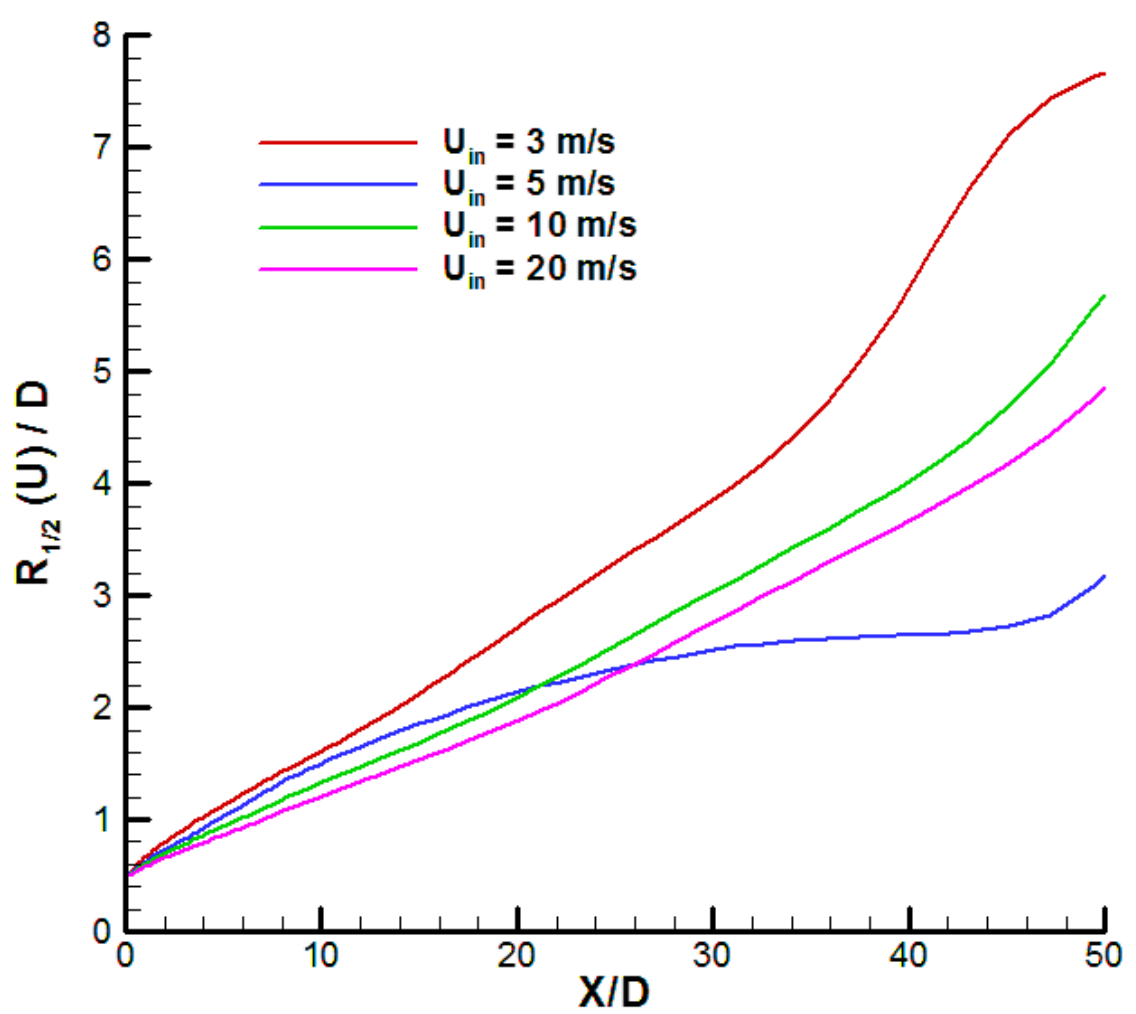

Figure 27 - Half width of half maximum of the velocity for the cases 4, 5, 6 and 7 of the Table 2 .

Finally, the half width of half maximum of the velocity is presented in Figure 27 for the test cases of Table 2. As it has been said in the previous subchapter these results allow the determination of the jet spreading angle. The spreading rate is determined through the calculation of the straight line's slope that defines the almost linear part of the graphic. The tangent of the spreading angle for the case 4 was determined previously having a value of 0.11261 . The half width of half maximum of the velocity for case 5 , with an injection velocity of $U_{\text {in }}=5 \mathrm{~m} . \mathrm{s}^{-1}$, does not show an initial almost linear part, however for comparison purposes, it was defined a straight line using the same $X / D$ points used in case 4 , and the tangent of the spreading rate calculated was 0.0504 . The graphic of the case 6, for $U_{\text {in }}=10 \mathrm{~m} \cdot \mathrm{s}^{-1}$, like the case 4 , has an almost linear part. The calculation of straight line's slope that defines the almost linear part of the graphic and represents the tangent of the spreading angle has the value of 0.0856 . 
The case 7 with an injection velocity of $U_{i n}=20 \mathrm{~m}_{\mathrm{s}} \mathrm{s}^{-1}$, follows the same trend observed for case 6 , and the graphic has the same evolution however with a lower value of the jet spreading rate. The tangent of the spreading angle calculated for this case was 0.0790 .

As for the results presented above, the injection velocity is one variable that shows to have a strong influence in the half width of half maximum of the velocity. Analyzing the Figure 27, manly cases 4, 6 and 7, we can conclude that this influence is made in the way of reducing the jet spreading angle as the injection velocity increases. Like in the previous two graphics the case 5 with an injection velocity of $U_{\text {in }}=5 \mathrm{~m} \cdot \mathrm{s}^{-1}$ shows not follow the same trend observed in the other cases.

The analysis of the results obtained in the injection velocity study allows the conclusion that in fact injection velocity influences the behavior of the jet. In general the increase of the injection velocity causes a slower change of the jet properties across the axial direction. Both dense and potential core of the jet growth and the rates of density and velocity decay as well as the jet spreading rate decrease. One possible explication for this behavior can be the fact that despite the increase of the injection velocity, the rate of change of the jet properties does not increase in the temporal reference in the same way that the velocity, and so, the same values of the jet properties are obtained further downstream as the jet fluid travels a greater distance in the same length of time.

The case 5 of Table 2 with an injection velocity of $U_{\text {in }}=5 \mathrm{~m} \cdot \mathrm{s}^{-1}$ appears in this study as an exception or an anomaly. 
Eduardo Luís Santos Farias Antunes 


\section{Chapter 4}

\section{Conclusion}

The present chapter exhibits the main conclusions achieved in this thesis.

Barata et al. [18] had concluded that the variable density mathematical model could be successfully used in the study of cryogenic jets injected initially at subcritical temperatures into an environment at a supercritical temperature over a range of subcritical and supercritical pressures [18]. The objective of the present work was to extend the study performed by Barata et al. [18] to lower chamber-to-injection fluid density ratios. Like in Barata et al. [18], for this investigation an injection of cryogenic liquid nitrogen into a gaseous nitrogen environment was simulated.

The major parameter of comparison between the results obtained in this work and others experimental and numerical works was the jet growth rate. It is important to refer that the computation model was not written for the study of liquid injections into gaseous ambient, but instead to study incompressible variable density gaseous jets. The test conditions imposed in the present work are completely outside of the range of application for which the computational model was though. However, it was conclude that for the three test cases the jet spreading angle obtained by the variable density approach are in general agreement with the experimental and modeling result of Chehroudi et al.[23]. These results show the capability of the incompressible variable density computational model to study cryogenic jets, injected initially at subcritical temperatures into an environment at a supercritical temperature over a range of subcritical pressures.

The study of conditions with lower density ratios allowed finding the limit of applicability of the incompressible variable density approach. It was verified that for density ratios bellow $\omega=0.025$ the computational model shows a dramatic change in the jet behavior and the results obtained for these conditions not fit with any experimental or numerical results obtained by other authors. This was the major conclusion achieved in the present thesis.

The study of the injection velocity in the jet behavior, despite being an investigation with less relevance in the present thesis, also allowed some interesting conclusions. It was found that for this computational model, a change in the injection velocity produces some differences in jet behavior. Moreover, it was verified a general trend that an increase in the injection velocity is responsible slower variation of the jet properties across the domain of study, however this conclusion is not observable in all the studied injection velocities. A further more complete analysis of the jet injection velocity influence in the jet behavior could be an interesting subject for a future investigation. 
Eduardo Luís Santos Farias Antunes 


\section{References}

[1] - Chehroudi, B., Cohn, R., and Talley, D., "Visual Characteristics and Initial Growth Rates of Round Cryogenic Jets at Subcritical and Supercritical Pressures," Physics of Fluids, Vol. 14, No. 2, 2002, pp. 850-861.

[2] - Bellan, J., "Supercritical (and Subcritical) Fluid Behavior and Modeling: Drops, Streams, Shear and Mixing Layers, Jets and Sprays," Progress in Energy and Combustion Science, Vol. 26, Issues 4-6, 2000, pp. 329-366.

[3] - Yang, V., "Modeling of Supercritical Vaporization, Mixing, and Combustion Processes in Liquid-Fueled Propulsion Systems," Proceedings Of the Combustion Institute, Vol. 28, 2000, pp. 925-942.

[4] - Segal, C., and Polikhov, S.A., "Subcritical to Supercritical Mixing," Physics of Fluids, Vol. 20, Issue 5, 2008, pp. 052101-1-052101-7.

[5] - Oschwald, M., Smith, J. J., Branam, R., Hussong, J., Schik, A., Chehroudi, B. and Talley, D. (2006) "Injection of Fluids into Supercritcal Environments," Combustion Science and Technology, Vol. 178, No. 1-3, 2006, pp. 49-100.

[6] - Mayer, W.O.H., Schick, A. H. A., Vielle, B., Chauveau, C., Gökalp, I., Talley, D.G., and Woodward, R.D., "Atomization and Breakup of Cryogenic Propellants under High-Pressure Subcritical and Supercritical Conditions," Journal of Propulsion and Power, Vol.14, No.5, 1998, pp.835-842.

[7] - Chehroudi, B., Cohn, R., and Talley, D., "Cryogenic Shear Layers: Experiments and Phenomenological Modeling of the Initial Growth Rate under Subcritical and Supercritical Conditions," International Journal of Heat and Fluid Flow, Vol. 23, Issue 5, 2002, pp. 554563.

[8] - Givler, S.D., John Abraham, J., "Supercritical Droplet Vaporization and Combustion Studies," Progress in Energy and Combustion Science, Vol. 22, Issue 1, 1996, pp. 1-28.

[9] - Zhang, H., Raghavan, V., Gogos, G., "Subcritical and Supercritical Droplet Evaporation within a Zero-Gravity Environment: Low Weber Number Relative Motion," International Communications in Heat and Mass Transfer, Vol. 35, Issue 4, 2008, pp. 385-394. 
[10] - Nomura, H., Ujiie, Y., Rath, H.J., Sato, J., Kono, M., "Experimental Study of HighPressure Droplet Evaporation using Microgravity Conditions," Twenty-Sixth Symposium (International) on Combustion/The Combustion Institute, 1996, pp. 1267-1273.

[11] - Tsue, M., Nomur, H., Niwa, M., Miyano, H., Sato, J., Kono, M., "Evaporation of a Fuel Droplet in a High Pressure High Temperature Atmosphere," Japan Society for Aeronautical and Space Sciences, Journal (ISSN 0021-4663), Vol. 37, No. 420, 1989, pp. 21-28.

[12] - Fieberg, C., Reichelt, L., Martin, D., Renz, U., and Kneer, R., "Experimental and Numerical Investigation of Droplet Evaporation under Diesel Engines Conditions," International Journal of Heat and Mass Transfer, Vol. 52, Issues 15-16, 2009, pp. 3738-3746.

[13] - Zhu, G.S., and Aggarwal, S.K., "Transient Supercritical Droplet Evaporation with emphasis on the Effects of Equation of State", International Journal of Heat and Mass Transfer, Vol. 43, Issue 7, 2000, pp. 1157-1171.

[14] - Emekwuru, N.G., Watkins, P., "Analysis of a Two-Fluid Sprayer and its Use to Develop the Number Size Distribution Moments Spray Model Part II: Computational Analysis," Atomization and Sprays, Vol. 20, Issue 8, 2010, pp. 653-672.

[15] - Oschwald, M., and Schik, A., "Supercritical Nitrogen Free Jet Investigated by Spontaneous Raman Scattering," Experiments in Fluids, Vol. 27, No. 6, 1999, pp. 497-506.

[16] - Mayer, W., and Telaar, J., "Investigation of Breakup of Turbulent Cryogenic VariableDensity Jets," Atomization and Sprays, Vol. 12, Issue 5-6, 2002, pp. 651-666.

[17] - Sanders, J. P. H., B. Sarh, B., Gokalp, I., “Variable Density Effects in Axisymmetric Isothermal Turbulent jets: a Comparison Between a First- and a Second-order Turbulence Model," Int. J. Heat Mass Transfer. Vol. 40, No. 4, 1997, pp. 823-842.

[18] - Barata, J.M.M., Gokalp, I., Silva, A.R.R., "Numerical Study of Cryogenic Jets under Supercritical Conditions," Journal of Propulsion and Power, Vol. 19, No.1, 2003, pp. 142-147.

[19] - Zong, N., Meng, H., Hsieh, S., and Yang, V., "A Numerical Study of Cryogenic Fluid Injection and Mixing under Supercritical Conditions," Physics of Fluids, Vol. 16, No. 12, 2004, pp. 4248-4261.

[20] - Sierra-Pallares, J., Parra-Santos, M.T., García-Serna, J., Castro, F., Cocero, M.J., "Numerical Analysis of High-pressure Fluid Jets: Application to RTD Prediction in Supercritical Reactors," Journal of Supercritical Fluids, Vol. 49, Issue 2, 2009, pp. 249-255. 
[21] - Schmitt, T., Selle, L., Cuenot, B., and Poinsot, T., "Large-Eddy Simulation of Transcritical Flows," Comptes Rendus Mécanique, Vol. 337, Issues 6-7, June-July 2009, pp. 528-538.

[22] - Kim, T., Kim, Y., Kim, S., "Numerical study of cryogenic liquid nitrogen jets at supercritical pressures," The Journal of Supercritical Fluids, Vol. 56, Issue 2, 2011, pp. 152 163.

[23] - Chehroudi, B., Cohn, R., and Talley, D., "Spray/Gas Behaviour of Cryogenic Fluids under Sub- and Supercritical Conditions," Eighth International Conference on Liquid Atomization \& Sprays Systems, ICLASS-2000, Pasadena, California, USA, July 16-20, 2000. 
Eduardo Luís Santos Farias Antunes 


\section{Attachment 1 \\ Extended Abstract send to AIAA}





\title{
Evaluation of Numerical Variable Density Approach to Cryogenic Jets
}

\author{
Eduardo L.S.F. Antunes ${ }^{1}$, André R. R. Silva ${ }^{2}$, Jorge M. M. Barata ${ }^{3}$ \\ Universidade da Beira Interior, Covilhã, 6201-1, Portugal
}

\begin{abstract}
Fuel injection presents itself as one of the great challenges in engineering of diesel engines, gas turbines and rocket engines, combining in the last one also the injection of oxidizer. It is widely known that the increase of operating pressure and temperatures in combustion chamber, or thrust chamber in rocket engines, leads to an increase of engine efficiency, reducing this way the fuel consumption. Thereby is a general trend in new engine designs to operate with higher chamber pressures and temperatures. Also the appearance of new and more resistant materials is other reason that could make grow this tendency. As a result of these increasingly higher pressures, the injected fluids may experience ambient conditions exceeding the critical values. The Space Shuttle Main Engine and the Vulcain with thrust chamber pressures of respectively 22.3 MPa and 28.2 MPa are both examples of engines in which the chamber pressure exceeds the critical pressure of $\mathrm{P}_{\mathrm{cr}}=5.043 \mathrm{MPa}$ for liquid oxygen and $\mathrm{P}_{\mathrm{cr}}=1.28 \mathrm{MPa}$ for liquid hydrogen ${ }^{1}$. In these application, the initial temperature of the oxygen can initially be below the critical temperature of the oxygen $\left(\mathrm{T}_{\mathrm{cr}}=154.58 \mathrm{~K}\right)$ but it then undergoes to a transition, when in the combustion chamber, reaching supercritical temperatures. At these conditions the liquid fuel is on supercritical conditions and its physical state is named as fluid. As the fluid reach pressure and temperature values exceeding the critical ones it suffers significantly changes in its properties. The effective mass diffusivity, the surface tension and the latent heat of the liquid all vanish in critical conditions. On the other hand, the heat capacity at constant pressure, $C \mathrm{p}$, the isentropic compressibility, $\kappa_{\mathrm{s}}$, and the thermal conductivity, $\lambda$, all become infinite [2]. These changes in the fluid behaviour cause the inapplicability of the traditional two-phase flow models used in fuel injection under subcritical conditions, thus there is a need to develop new models with can correctly be applied to supercritical fuel injection.

Several authors investigated the fuel injection in supercritical condition both in experimentally and numerically ${ }^{3-22}$. The first experimental investigations performed had as principal objective the study of the visual structure of the jet without obtaining any quantitative result ${ }^{1,6}$, these investigations observed that the jet structure suffers significantly changes as the pressure increases, firstly the surface tension reduction leads to the formations of jet ligaments and droplets that detach from the main jet structure and a further increase of the chamber pressure into supercritical conditions leads to gas-gas like structure of the jet. More recent experimental work made quantitative studies in which results like the jet spreading angle, density and temperature were obtained ${ }^{4,7,15,16}$. These quantitative experimental results allowed the comparison with the results obtained in numerical studies and this way validate the numerical models ${ }^{17-22}$.

Barata et al. ${ }^{18}$ performed an initial investigation aimed to evaluate the capabilities of a computational method developed for incompressible but variable density flows when applied to supercritical conditions. Their results have show a good agreement with the experimental data, but they only considered intermediate density ratios from 0.05 to 0.14 . The present work extend the investigation of Ref. 18 to much lower density ratios and investigates the limits of application of the variable density approach to supercritical jets.

The flow configuration is the same used before experimentally ${ }^{1,7}$ and numerically ${ }^{18}$ and is shown in Figure 1. A cryogenic nitrogen jet $\left(\mathrm{LN}_{2}\right)$ is injected initially at subcritical temperature of $100-110 \mathrm{~K}$ into an environment at an ambient temperature $(300 \mathrm{~K})$ gaseous nitrogen $\left(\mathrm{GN}_{2}\right)$ but in this work is only over a range of subcritical pressures close to the critical value.

The reduced pressure $\operatorname{Pr}$ which is defined as the ratio of the chamber pressure to the critical pressure $\left(\mathrm{P}_{\text {critical }}=3.39 \mathrm{Mpa} ; \mathrm{T}_{\text {critical }}=126.2 \mathrm{~K}\right)$ in this work is change between $\operatorname{Pr}=0.583$ and $\operatorname{Pr}=0.825$.

The computational method was developed to solve variable density jet flows, and is based on the solution of the conservation equations for momentum and mass. Turbulence is modelled with the " $\mathrm{k} \sim \mathrm{\varepsilon}$ " turbulence model. In the conservation equations, mass weighted averaging is applied to avoid the appearance of many terms involving density fluctuations for which additional models are needed. To describe mixing of gases, the mixture fraction $F$, which represents the mass fraction of the nozzle fluid, is
\end{abstract}

\footnotetext{
${ }^{1}$ MEng Student, Aerospace Sciences Department, Rua Marques Avila e Bolama.

${ }^{2}$ Assistant Professor, Aerospace Sciences Department, AIAA Member.

${ }^{3}$ Full Professor, Aerospace Sciences Department, Associate Fellow of AIAA.
} 
introduced. The approach adopted here has been applied to variable density jets and its performance and limits of applicability are evaluated for lower density ratios.

The typical velocity and scalar field of the jet for lower density ratios are shows in Figure 2. For this figure was chosen the case with a density ratio of $\omega=0.025$ which is the studied case with the lower density ratio. This case corresponds to a liquid nitrogen injection at a temperature of $100 \mathrm{~K}$ into an environment at a pressure of $0.583 \mathrm{P}_{\mathrm{cr}}$ and a temperature of $300 \mathrm{~K}$. The velocity field shows the formation of a vortex above the jet and some divergence of the jet is visible after a length of approximately $0.004 \mathrm{~m}$ due to the vortex. The density suffers a rapid decrease near the injector with the jet reaching the ambience density in a very small length, the mixture fraction also shows a rapid decrease, although slower than the density decrease.

The Figure 3 shows the axial velocity for the tree different chamber pressures, it is observed bigger velocity decay for de case with higher chamber pressure, this can indicate a smaller jet penetration in the case with higher pressure.

The jet spreading is determined by the full width at half maximum of velocity profile and it is showed in Figure 4. Numerical results show a slightly decrease of the jet spreading angle as the pressure increases in the subcritical pressure ranges.

Figure 5 shows the influence of the chamber-to-injectant density ratio into jet spray or mixing layer divergence angle. The results obtained agree relatively well with both the experimental measurements for the $\mathrm{LN}_{2}$ injection into $\mathrm{GN}_{2}$ environment. The present results are also shown in Figure 5, and revel that the variable density approach does not offers valid data for density ratios below $\omega=0.025$.

\section{References}

${ }^{1}$ Chehroudi, B., Cohn, R., and Talley, D., "Visual Characteristics and Initial Growth Rates of Round Cryogenic Jets at Subcritical and Supercritical Pressures," Physics of Fluids, Vol. 14, No. 2, 2002, pp. 850-861.

${ }^{2}$ Bellan, J., "Supercritical (and Subcritical) Fluid Behavior and Modeling: Drops, Streams, Shear and Mixing Layers, Jets and Sprays," Progress in Energy and Combustion Science, Vol. 26, Issues 4-6, 2000, pp. 329-366.

${ }^{3}$ Yang, V., "Modeling of Supercritical Vaporization, Mixing, and Combustion Processes in Liquid-Fueled Propulsion Systems," Proceedings Of the Combustion Institute, Vol. 28, 2000, pp. 925-942.

${ }^{4}$ Segal, C., and Polikhov, S.A., "Subcritical to Supercritical Mixing," Physics of Fluids, Vol. 20, Issue 5, 2008, pp. 052101-1-052101-7.

${ }^{5}$ Oschwald, M., Smith, J. J., Branam, R., Hussong, J., Schik, A., Chehroudi, B. and Talley, D. (2006) "INJECTION OF FLUIDS INTO SUPERCRITICAL ENVIRONMENTS", Combustion Science and Technology, 178: $1,49-100$

${ }^{6}$ Mayer, W.O.H., Schick, A. H. A., Vielle, B., Chauveau, C., Gökalp, I., Talley, D.G., and Woodward, R.D., "Atomization and Breakup of Cryogenic Propellants under High-Pressure Subcritical and Supercritical Conditions," Journal of Propulsion and Power, Vol.14, No.5, 1998, pp.835-842.

${ }^{7}$ Chehroudi, B., Cohn, R., and Talley, D., "Cryogenic Shear Layers: Experiments and Phenomenological Modeling of the Initial Growth Rate under Subcritical and Supercritical Conditions," International Journal of Heat and Fluid Flow, Vol. 23, Issue 5, 2002, pp. 554-563.

${ }^{8}$ Givler, S.D., John Abraham, J., "Supercritical Droplet Vaporization and Combustion Studies," Progress in Energy and Combustion Science, Vol. 22, Issue 1, 1996, pp. 1-28.

${ }^{9}$ Zhang, H., Raghavan, V., Gogos, G., "Subcritical and Supercritical Droplet Evaporation within a Zero-Gravity Environment: Low Weber Number Relative Motion," International Communications in Heat and Mass Transfer, Vol. 35, Issue 4, 2008, pp. 385-394.

${ }^{10}$ Nomura, H., Ujiie, Y., Rath, H.J., Sato, J., Kono, M., "Experimental Study of High-Pressure Droplet Evaporation using Microgravity Conditions," Twenty-Sixth Symposium (International) on Combustion/The Combustion Institute, 1996, pp. 1267-1273.

${ }^{11}$ Tsue, M., Nomur, H., Niwa, M., Miyano, H., Sato, J., Kono, M., "Evaporation of a Fuel Droplet in a High Pressure High Temperature Atmosphere," Japan Society for Aeronautical and Space Sciences, Journal (ISSN 00214663), Vol. 37, No. 420, 1989, pp. 21-28.

${ }^{12}$ Fieberg, C., Reichelt, L., Martin, D., Renz, U., and Kneer, R., "Experimental and Numerical Investigation of Droplet Evaporation under Diesel Engines Conditions," International Journal of Heat and Mass Transfer, Vol. 52, Issues 15-16, 2009, pp. 3738-3746.

${ }^{13}$ Zhu, G.S., and Aggarwal, S.K., "Transient Supercritical Droplet Evaporation with emphasis on the Effects of Equation of State", International Journal of Heat and Mass Transfer, Vol. 43, Issue 7, 2000, pp. 1157-1171.

${ }^{14}$ Emekwuru, N.G., Watkins, P., "Analysis of a Two-Fluid Sprayer and its Use to Develop the Number Size Distribution Moments Spray Model Part II: Computational Analysis," Atomization and Sprays, Vol. 20, Issue 8 , 2010, pp. 653-672.

${ }^{15}$ Oschwald, M., and Schik, A., "Supercritical Nitrogen Free Jet Investigated by Spontaneous Raman Scattering," Experiments in Fluids, Vol. 27, No. 6, 1999, pp. 497-506.

${ }^{16}$ Mayer, W., and Telaar, J., "Investigation of Breakup of Turbulent Cryogenic Variable-Density Jets," Atomization and Sprays, Vol. 12, Issue 5-6, 2002, pp. 651-666.

${ }^{17}$ Sanders, J. P. H., B. Sarh, B., Gokalp, I., "Variable Density Effects in Axisymmetric Isothermal Turbulent jets: a Comparison Between a First- and a Second-order Turbulence Model," Int. J. Heat Mass Transfer. Vol. 40, No. 4 1997, pp. 823-842. 
${ }^{18}$ Barata, J.M.M., Gokalp, I., Silva, A.R.R., "Numerical Study of Cryogenic Jets under Supercritical Conditions," Journal of Propulsion and Power, Vol. 19, No.1, 2003, pp. 142-147.

${ }^{19}$ Zong, N., Meng, H., Hsieh, S., and Yang, V., "A Numerical Study of Cryogenic Fluid Injection and Mixing under Supercritical Conditions," Physics of Fluids, Vol. 16, No. 12, 2004, pp. 4248-4261.

${ }^{20}$ Sierra-Pallares, J., Parra-Santos, M.T., García-Serna, J., Castro, F., Cocero, M.J., "Numerical Analysis of Highpressure Fluid Jets: Application to RTD Prediction in Supercritical Reactors", Journal of Supercritical Fluids, Vol. 49, 2009, pp. 249-255.

${ }^{21}$ Schmitt, T., Selle, L., Cuenot, B., and Poinsot, T., "Large-Eddy Simulation of Transcritical Flows", Comptes Rendus Mécanique, Vol. 337, Issues 6-7, June-July 2009, pp. 528-538.

${ }^{22}$ Kim, T., Kim, Y., Kim, S., "Numerical study of cryogenic liquid nitrogen jets at supercritical pressures," The Journal of Supercritical Fluids, Vol. 56, Issue 2, 2011, pp. 152-163.

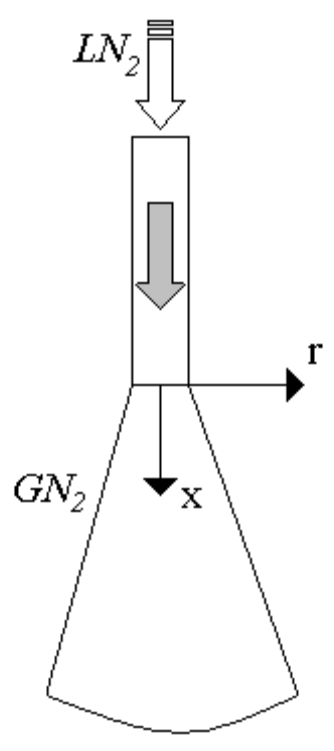

Figure 1. Flow configuration 
a)

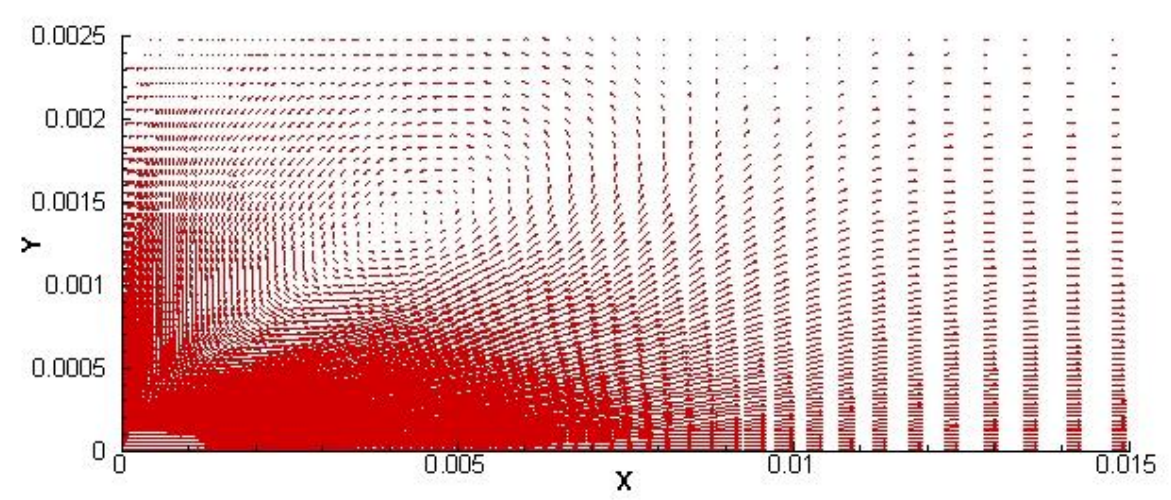

b)

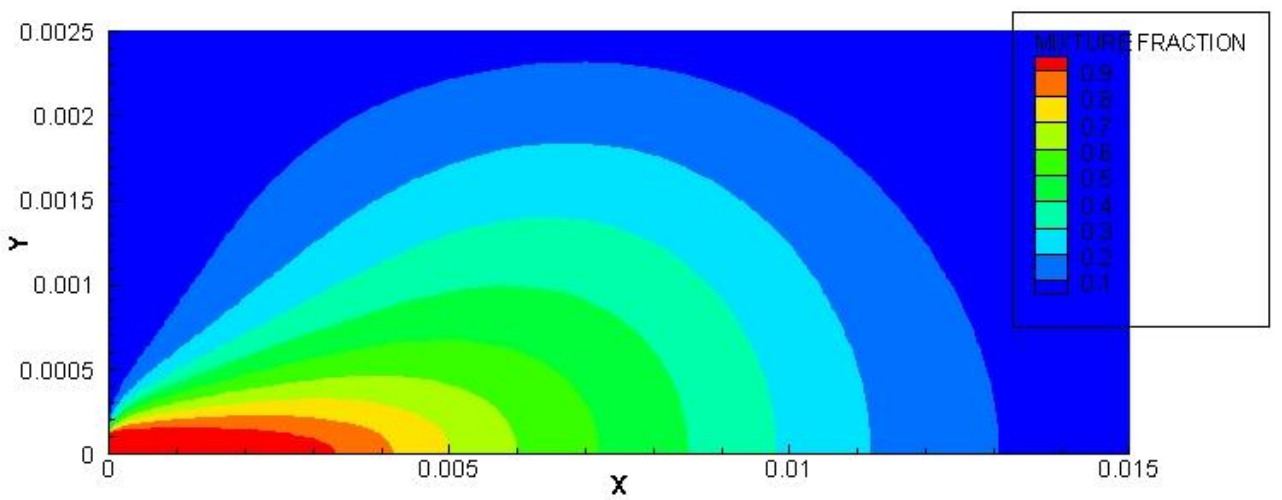

c)

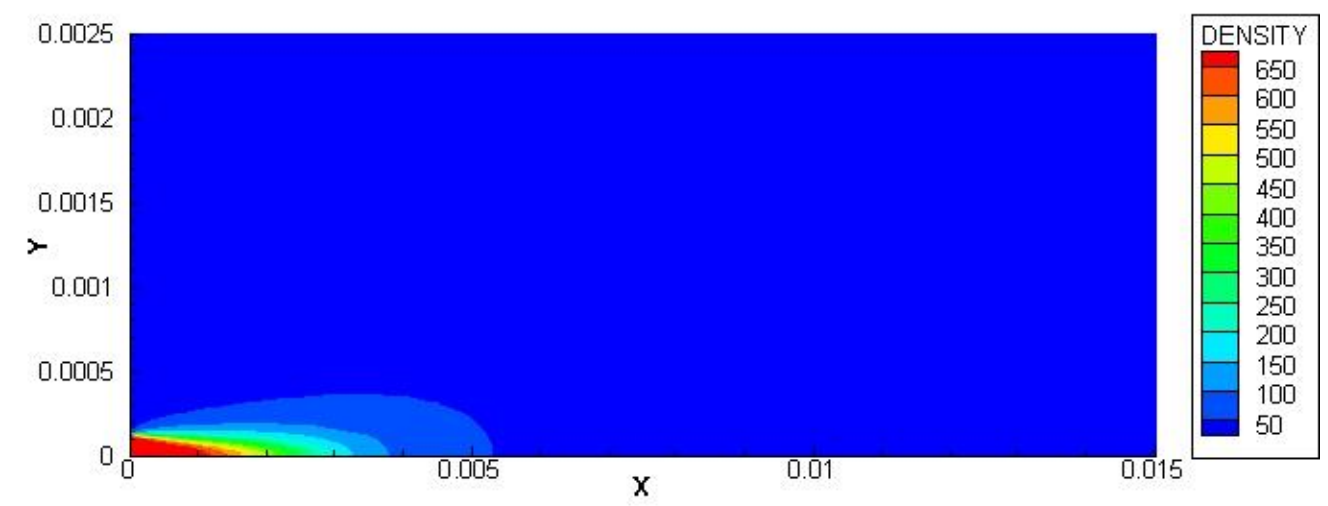

Figure 2. Velocity and scalar fields of the jet with a density ratio of $\omega=0.025$ and a chamber pressure of $\operatorname{Pr}=\mathbf{0 . 5 8 3}$, (a) Velocity vectors, (b) Mixture fraction contours, (c) Density contours. 


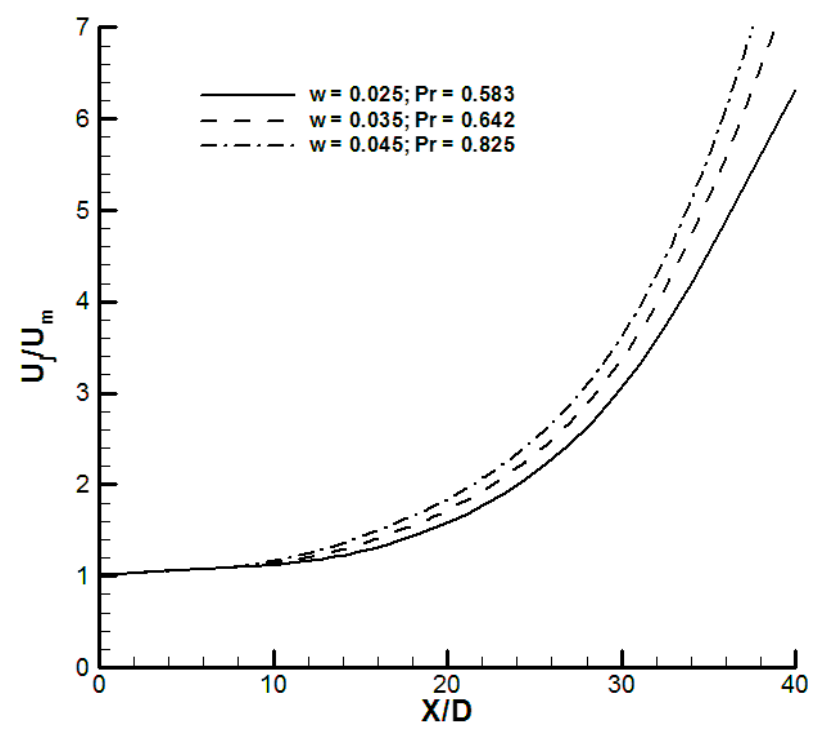

Figure 3. Non-dimensional profile of the mean axial velocity, $U$.

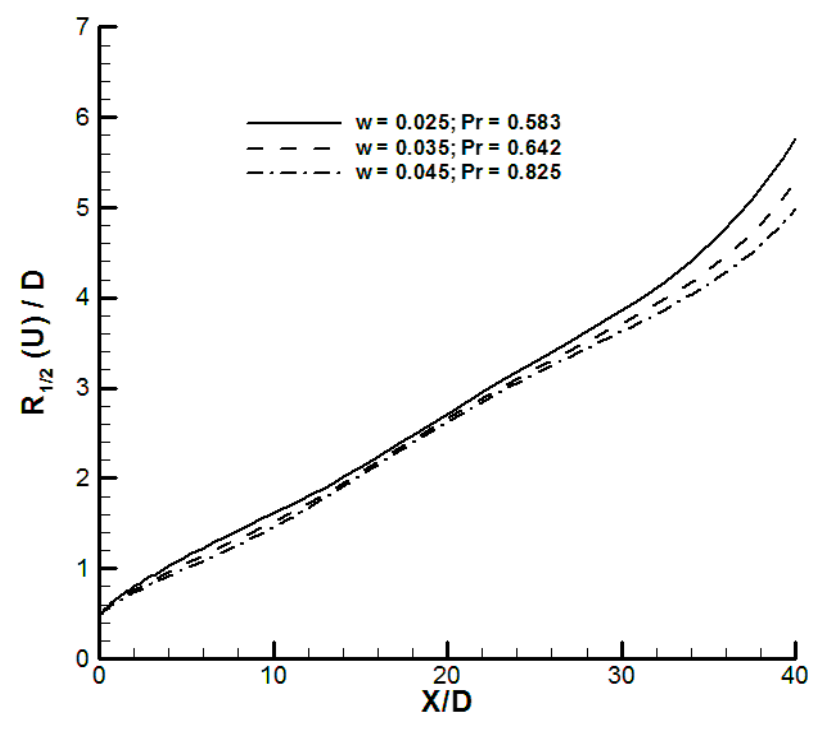

Figure 4. Full width at half maximum of velocity profile. 


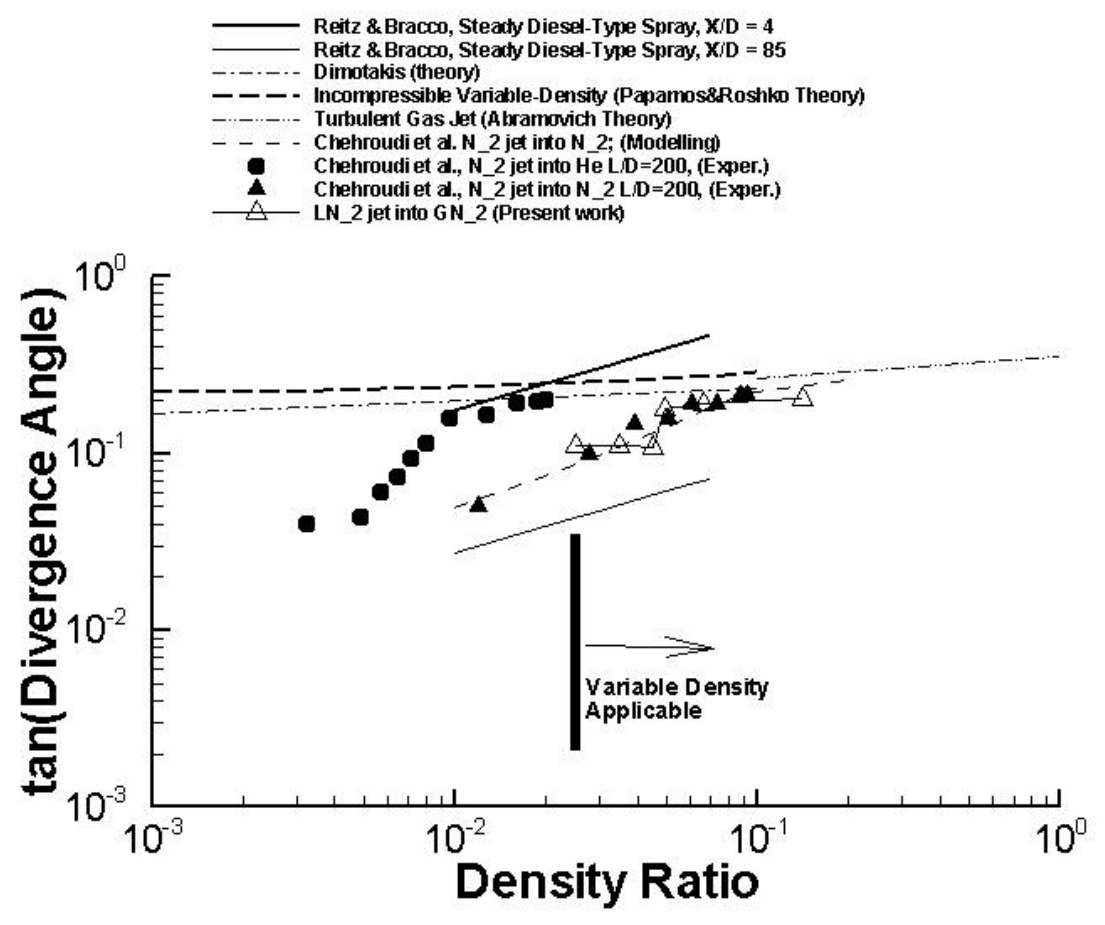

Figure 5. Tangent of the spreading angle versus the chamber-to-injectant density ratio and representation if limit of applicability of the Variable Density approach. 


\section{Attachment 2 \\ Paper send to the International Conference on Engineering UBI2011}





\title{
Application of a Numerical Variable Density Method to cryogenic jets
}

\author{
E.L.S.F. Antunes ${ }^{1}$, A. R. R. Silva ${ }^{2}$, J. M. M. Barata ${ }^{3}$ \\ Universidade da Beira Interior, 6200-001 Covilhã, Portugal \\ Conference Topic - CT 1
}

\begin{abstract}
The present work is devoted to study cryogenic nitrogen jets in high subcritical conditions. It is a general trend in modern engines the operation in increasingly higher pressures which enhance efficiency. However at higher chamber pressures the injected fluids may experience ambient conditions exceeding the critical values and changing their properties, thus requiring new computational models. Barata et al. [18] performed a numerical investigation evaluating the applicability of an incompressible but variable density model in liquid jets under sub-to-supercritical conditions. The results achieved agree well with the experimental data but they only considered intermediate density ratios. This work extends the investigation of Ref. 18 to lower density ratios. The obtained results agree well with the experimental and numerical data of Chehroudi et al. presented in Ref. 18. It was also found in this work that the computational model does not offers valid results for density ratios below $\omega=0.025$.
\end{abstract}

Keywords: Fuel injection; critical point; supercritical conditions; rocket engines; diesel engines; density ratio; jets; variable density.

\section{Nomenclature}

$B \quad=$ transfer number

$B_{v} \quad=$ evaporation rate

$C_{\mu} \quad=$ coefficient in turbulence model

$d_{0} \quad=$ initial droplet diameter

$d \quad=$ droplet diameter

$D \quad=$ injector diameter [m], normalized droplet diameter $\left(\mathrm{d}(\mathrm{t}) / \mathrm{d}_{0}\right)$

$\varepsilon \quad=$ dissipation rate of turbulent energy

$f \quad=$ mixture fraction

$F \quad=$ mean mixture fraction

$i \quad=$ axial direction index

$j \quad=$ radial direction index

$k=$ turbulent kinetic energy

$\phi \quad=$ generalized variable

$\omega=$ chamber-to-injection fluid density ratio $\left(\rho_{\infty} / \rho_{0}\right)$

$P_{c r} \quad=$ critical pressure $[\mathrm{MPa}]$

$P_{\infty} \quad=$ chamber ambient pressure [MPa]

$P_{r} \quad=$ reduced pressure $\left(\mathrm{P}_{\infty} / \mathrm{P}_{\mathrm{cr}}\right)$

$\rho=$ density $\left[\mathrm{kg} \cdot \mathrm{m}^{-3}\right]$

$\rho_{0} \quad=$ injected fluid density $\left[\mathrm{kg} . \mathrm{m}^{-3}\right]$

$\rho_{\infty} \quad=$ injection chamber's fluid density $\left[\mathrm{kg} \cdot \mathrm{m}^{-3}\right]$

$r \quad=$ radial coordinate $[\mathrm{m}]$
${ }^{3}$ Full Professor, Aerospace Sciences Department, AIAA Associate Fellow.

$R_{\text {diam }}=$ injector radius [m]

$R / D \quad=$ radial distance normalized by

injector diameter

$R_{e} \quad=$ Reynolds Number

$S_{\phi} \quad=$ source term

$t=$ time [s]

$T \quad=$ temperature $[\mathrm{K}]$

$u=$ axial velocity $\left[\mathrm{m} . \mathrm{s}^{-1}\right]$

$U \quad=$ mean axial velocity $\left[\mathrm{m} . \mathrm{s}^{-1}\right]$

$U_{i n} \quad=$ injection axial velocity $\left[\mathrm{m} . \mathrm{s}^{-1}\right]$

$v \quad=$ radial velocity $\left[\mathrm{m} . \mathrm{s}^{-1}\right]$

$v_{t} \quad=$ turbulent kinematic viscosity

$V \quad=$ mean radial velocity $\left[\mathrm{m} . \mathrm{s}^{-1}\right]$

$X=$ axial coordinate $[\mathrm{m}]$

$X / D=$ axial distance normalized by

injector diameter

\section{Introduction}

Fuel injection presents itself as one of the great challenges in engineering of diesel engines, gas turbines and rocket engines, combining in the last one also the injection of oxidizer. It is widely known that the increase of operating pressure and temperatures in combustion chamber, or thrust chamber in rocket engines, leads to an increase of engine efficiency, reducing this way the fuel consumption. Thereby is a general trend in new engine designs to operate with higher chamber pressures and temperatures. Also the appearance of new and more resistant materials is other reason that could make grow this tendency. As a 
result of these increasingly higher pressures, the injected fluids may experience ambient conditions exceeding the critical values. The Space Shuttle Main Engine and the Vulcain with thrust chamber pressures of respectively 22.3 $\mathrm{MPa}$ and 28.2 $\mathrm{MPa}$ are both examples of engines in which the chamber pressure exceeds the critical pressure of $\mathrm{P}_{\mathrm{cr}}=$ $5.043 \mathrm{MPa}$ for liquid oxygen and $\mathrm{P}_{\mathrm{cr}}=1.28$ $\mathrm{MPa}$ for liquid hydrogen ${ }^{1}$. In these application, the initial temperature of the oxygen can initially be below the critical temperature of the oxygen $\left(\mathrm{T}_{\mathrm{cr}}=154.58 \mathrm{~K}\right)$ but it then undergoes to a transition, when in the combustion chamber, reaching supercritical temperatures. At these conditions the liquid fuel is on supercritical conditions and its physical state is named as fluid. As the fluid reach pressure and temperature values exceeding the critical ones, it suffers significantly changes in its properties. The effective mass diffusivity, the surface tension and the latent heat of the liquid all vanish in critical conditions. On the other hand, the heat capacity at constant pressure, $\mathrm{Cp}$, the isentropic compressibility, $\mathrm{K}_{\mathrm{s}}$, and the thermal conductivity, $\lambda$, all become infinite [2]. These changes in the fluid behavior cause the inapplicability of the traditional two-phase flow models used in fuel injection under subcritical conditions, thus there is a need to develop new models with can correctly be applied to supercritical fuel injection.

Several authors investigated the fuel injection in supercritical condition both in experimentally and numerically ${ }^{3-22}$. The first experimental investigations performed used techniques like photography and shadowgraphy, and had as principal objective the study of the visual structure of the jet without obtaining any quantitative result $t^{1,6}$, these investigations observed that the jet structure suffers significantly changes as the pressure increases, firstly the surface tension reduction leads to the formations of jet ligaments and droplets that detach from the main jet structure and a further increase of the chamber pressure into supercritical conditions leads to gas-gas like structure of the jet. In more recent experimental works, along with qualitative characterization, were made quantitative studies in which results like the jet spreading angle, density and temperature were obtained ${ }^{4,7,15,16}$. These quantitative experimental results allowed the comparison with the results obtained in numerical studies and this way validate the numerical models ${ }^{17-22}$.
Barata et al. ${ }^{18}$ performed an initial investigation aimed to evaluate the capabilities of a computational method developed for incompressible but variable density flows when applied to supercritical conditions. Their results have show a good agreement with the experimental data, but they only considered intermediate density ratios from 0.05 to 0.14 . The present work extends the investigation of Ref. 18 to lower density ratios, corresponding to sub/nearcritical conditions, and investigates the limits of application of the variable density approach to supercritical jets. An injection velocity study was also performed in the present work with intend of better characterize the variable density approach.

\section{Mathematical Model}

\section{Governing Equations}

The method to solve variable density jet flows is based on the solution of the conservation equations for momentum and mass. Turbulence is modeled with the " $k \sim \varepsilon$ " turbulence model. A similar method has been used for three-dimensional or axisymmetric flows and only the main features are summarized here.

In the conservation equations, mass weighted averaging is applied to avoid the appearance of many terms involving density fluctuations for which additional models are needed. A mass averaged quantity is defined as

$$
\tilde{\phi}=\frac{\overline{\rho \phi}}{\bar{\rho}} .
$$

For the governing equations the standard parabolic truncation is employed. The mass averaged partial differential equations governing the steady, variable density axisymmetric flow may be written in cylindrical polar coordinates as

$$
\begin{gathered}
\frac{\partial \bar{\rho} U U}{\partial x}+\frac{1}{r} \frac{\partial r \bar{\rho} U V}{\partial r}=-\frac{\partial \bar{p}}{\partial x}-\frac{1}{r} \frac{\partial r \bar{\rho} \frac{\tilde{\rho}}{\partial u^{\prime} v^{\prime}}}{\partial r} \\
\frac{\partial \bar{\rho} U V}{\partial x}+\frac{1}{r} \frac{\partial r \bar{\rho} V V}{\partial r}=-\frac{\partial \bar{p}}{\partial x}-\frac{1}{r} \frac{\partial r \bar{\rho} \frac{\tilde{v^{\prime} v^{\prime}}}{\partial r}+\bar{\rho} \frac{\tilde{w^{\prime} w^{\prime}}}{r}}{}
\end{gathered}
$$


and the continuity equation as

$$
\frac{\partial \bar{\rho} U}{\partial x}+\frac{1}{r} \frac{\partial r \bar{\rho} V}{\partial r}=0
$$

To describe mixing of gases, the mixture fraction $F$, that represents the mass fraction of the nozzle fluid, is introduced. It obeys a convection-diffusion equation of the form

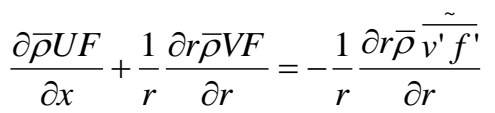

In " $\mathrm{k} \sim \varepsilon$ " turbulence model, the Reynolds stresses are expressed in terms of the local strain rate:

$$
-\bar{\rho} \overline{u_{i}^{\prime} u_{j}^{\prime}}=\bar{\rho}\left(v_{t}+v\right)\left(\frac{\partial \tilde{u}_{i}}{\partial x_{j}}+\frac{\partial \tilde{u}_{j}}{\partial x_{i}}\right)-\frac{2}{3} \delta_{i j}\left(\bar{\rho} k+\bar{\rho}\left(v_{t}+v\right) \frac{\partial \tilde{u}_{j}}{\partial x_{j}}\right)
$$

with

$$
v_{t}=C_{\mu} \frac{k^{2}}{\varepsilon}
$$

The scalar flux in equation (5) is approximated with a gradient transport assumption

$$
\overline{u_{i}^{\prime} f^{\prime}}=-\frac{v_{t}}{\sigma_{f}} \frac{\partial F}{\partial x_{i}}
$$

From the foregoing we can deduced the parabolized set of equations in cylindrical coordinates where the generalized equation is

$$
\frac{\partial}{\partial x}(\bar{\rho} U \tilde{\phi})+\frac{1}{r} \frac{\partial}{\partial r}(r \bar{\rho} \tilde{\phi})=\frac{1}{r} \frac{\partial}{\partial r}\left(r \bar{\rho} D \frac{\partial \tilde{\phi}}{d r}\right)+S_{\phi}
$$

where $\tilde{\phi}$ may stand for any of the velocities, turbulent kinetic energy, dissipation, or scalar property, and $S_{\phi}$ take on different values for each particular $\tilde{\phi}$, described in detail by Sanders et $\mathrm{al}^{17}$.
The mean density can be obtained from the mean mixture fraction using the equation state. With constant pressure this leads to

$$
\frac{1}{\bar{\rho}}=\frac{F}{\rho_{o}}+\frac{1-F}{\rho_{\infty}}
$$

where density fluctuations have been neglected. This is allowed in isothermal jets because the instantaneous density, for which equation (10) is exact, is approximately a linear function of the instantaneous mixture fraction ${ }^{18}$.

\section{Numerical Method}

The governing equations are solved using a parabolized marching algorithm which resembles the (elliptic) TEACH code [17]. The computations are performed by using the continuity equation to obtain the radial velocity $(V)$. Using the radial momentum equation for $V$ and solving a pressure correction equation for $V$ in radial direction did not show any difference with the use of the continuity equation [17]. In this approach the numerical model was applied to variable density jets and for the present case it was used for the study of liquid cryogenic jets under

In order to determine the tangent of the jet spreading angle is used the Half Width of Half Maximum of the Velocity (HWHMV), this routine has the function of determine the radial distance from the centerline $(r)$ at which the axial mean velocity $(U)$ is half of the maximum velocity localized in the centerline. This routine analysis all the points in the radial direction comparing them with half of the centerline velocity, when the routine finds a point bellow this value it saves the point before that.

\section{Boundary Conditions}

There are four boundaries in the computational domain, in these boundaries dependent variables are specified: an inlet and outlet plane, a symmetry axis and a free boundary parallel to the axis. The sensitivity of the solutions to the locations of the boundaries was investigated in previous works, and their final position is sufficiently far away from the jet so that the influence on the computed results is negligible [18]. At the inlet boundary the $1 / 7$ th power law turbulent velocity profile was used for the axial velocity at the jet exit. 


$$
U=U_{\text {in }} \cdot\left(1-\frac{r}{R_{\text {diam }}}\right)^{\frac{1}{7}}
$$

The radial velocity is zero at the jet exit and in ambient. The mixture fraction is one at the jet exit and zero in the ambient. On the symmetry axis, the normal velocity vanishes, and the normal derivatives of the other variables are zero. At the outflow boundary, the gradients of dependent variables in the axial direction are set to zero.
The flow configuration can be observed in Figure 1. The injector nozzle has a diameter of $2.54 \times 10^{-4} \mathrm{~m}$ while the domain of study has an axial length of $1.77 \times 10^{-2} \mathrm{~m}$ and a radial length of $3.49 \times 10^{-3} \mathrm{~m}$. The test conditions for the present study of different densities ratios are presented in Table 1.

It was also performed a parallel study with the objective of evaluate the numerical model for different injection velocities, the test conditions used are summarized in Table 2.

Table 3. Summary of test conditions

\begin{tabular}{|c|c|c|c|c|c|c|c|}
\hline Case & $\operatorname{Pr}$ & $U_{\text {in }}[\mathrm{m} / \mathrm{s}]$ & $\operatorname{Re}$ & $\mathrm{T}_{0}[\mathrm{~K}]$ & $\begin{array}{c}\rho_{0} \\
{\left[\mathrm{Kg} / \mathrm{m}^{3}\right]}\end{array}$ & $\begin{array}{c}\rho_{\infty} \\
{\left[\mathrm{Kg} / \mathrm{m}^{3}\right]}\end{array}$ & $\omega$ \\
\hline 1 & 0,583 & 3.0 & 72060 & 100 & 700 & 17,5 & 0,025 \\
\hline 2 & 0,642 & 3.0 & 72060 & 100 & 700 & 24,5 & 0,035 \\
\hline 3 & 0,825 & 3.0 & 72060 & 100 & 700 & 31,5 & 0,045 \\
\hline
\end{tabular}

Table 4. Summary of test conditions for the injection velocity study

\begin{tabular}{|c|c|c|c|c|c|c|c|}
\hline Case & $\operatorname{Pr}$ & $\mathrm{U}_{\text {in }}[\mathrm{m} / \mathrm{s}]$ & $\operatorname{Re}$ & $\mathrm{T}_{0}[\mathrm{~K}]$ & $\begin{array}{c}\rho_{0} \\
{\left[\mathrm{Kg} / \mathrm{m}^{3}\right]}\end{array}$ & $\begin{array}{c}\rho_{\infty} \\
{\left[\mathrm{Kg} / \mathrm{m}^{3}\right]}\end{array}$ & $\omega$ \\
\hline 4 & 0,583 & 3.0 & 72060 & 100 & 700 & 17,5 & 0,025 \\
\hline 5 & 0,583 & 5.0 & 120100 & 100 & 700 & 17,5 & 0,025 \\
\hline 6 & 0,583 & 10.0 & 240200 & 100 & 700 & 17,5 & 0,025 \\
\hline 7 & 0,583 & 20.0 & 480400 & 100 & 700 & 17,5 & 0,025 \\
\hline
\end{tabular}

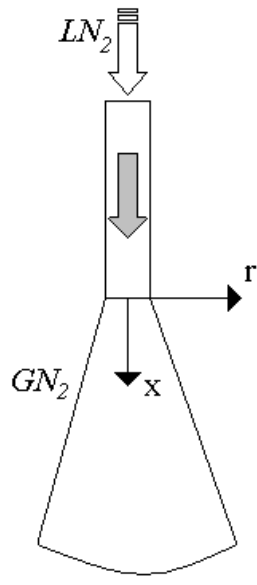

Figure 1 - Flow Configuration

\section{Results and discussion}

In this section are presented the numerical results obtained at the present work and compared with the results exposed by Barata et al. ${ }^{18}$. The main objective is to extend the analysis performed by Barata et al. ${ }^{18}$ to lower density ratios and then compare them with the experimental data in order to investigate the applicability of the variable density approach to liquid jet injection into a gaseous ambient under subcritical chamber pressures but supercritical temperatures. The parameter used to compare the numerical results obtained in the present work with the results 
a)

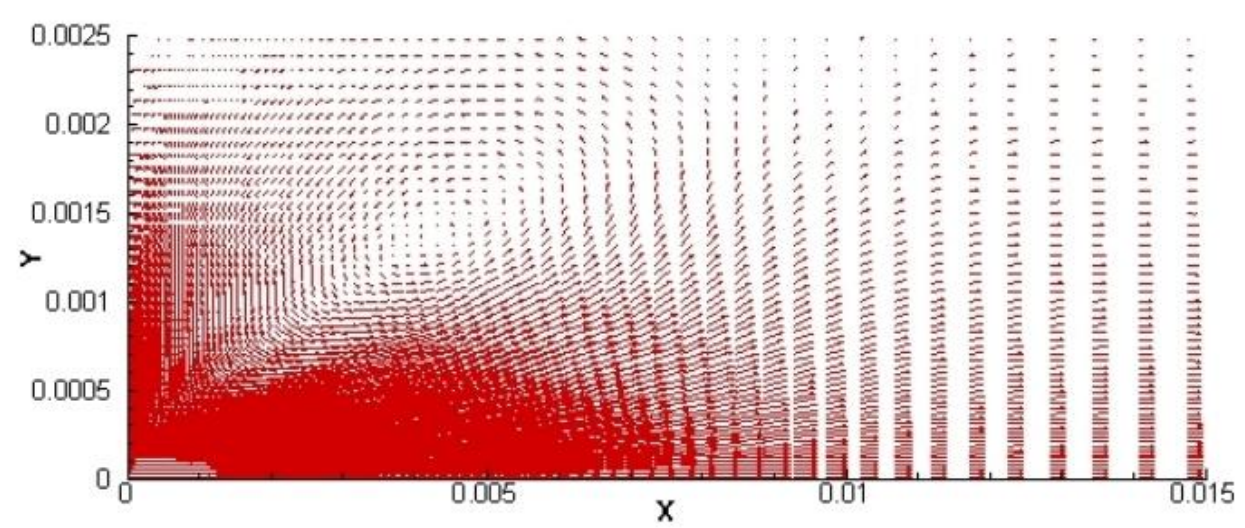

b)

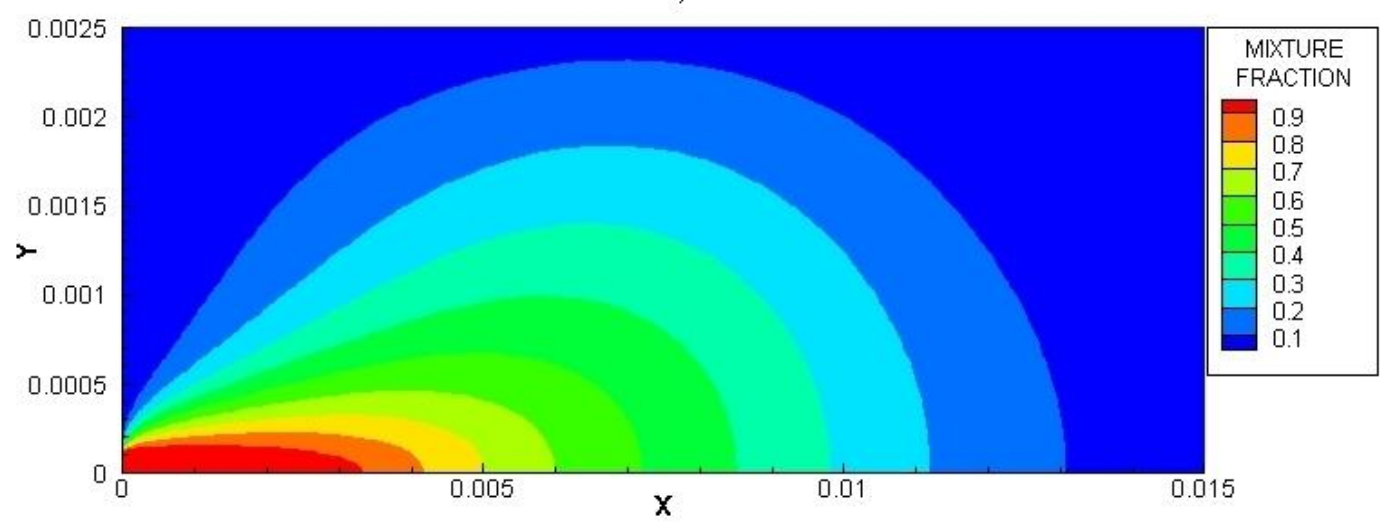

c)

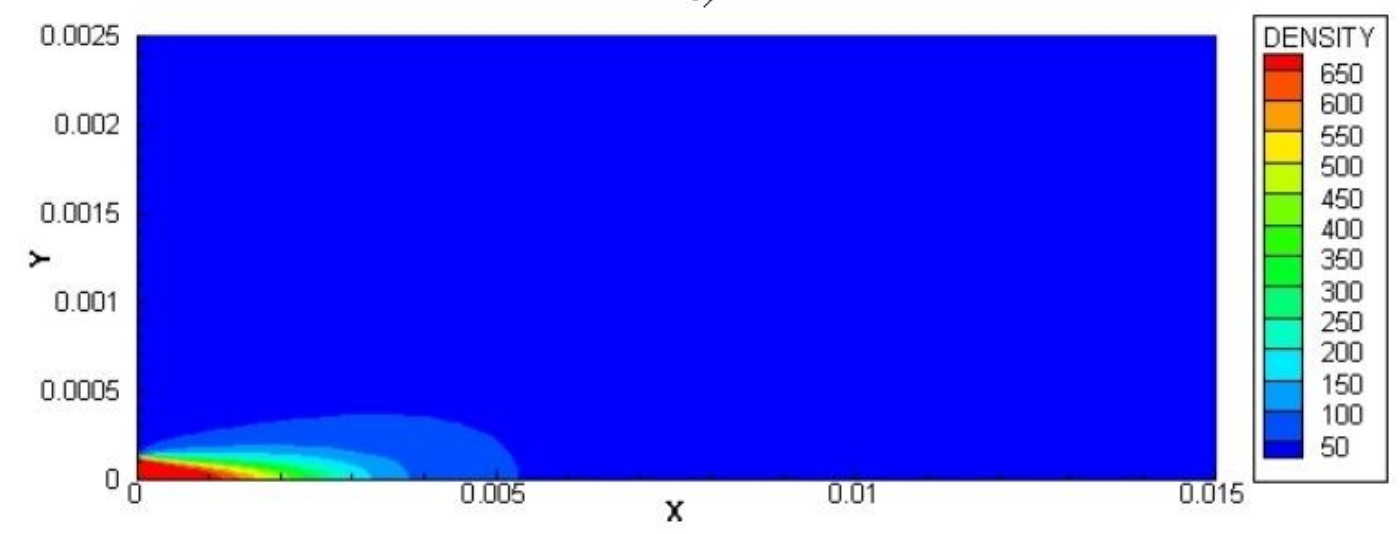

Figure 2 - Velocity and scalar fields of the jet with a density ratio of $\omega=0.025$ and a chamber pressure of $\operatorname{Pr}=0.583$, (a) Velocity vectors, (b) Mixture fraction contours, (c) Density contours. 
a)

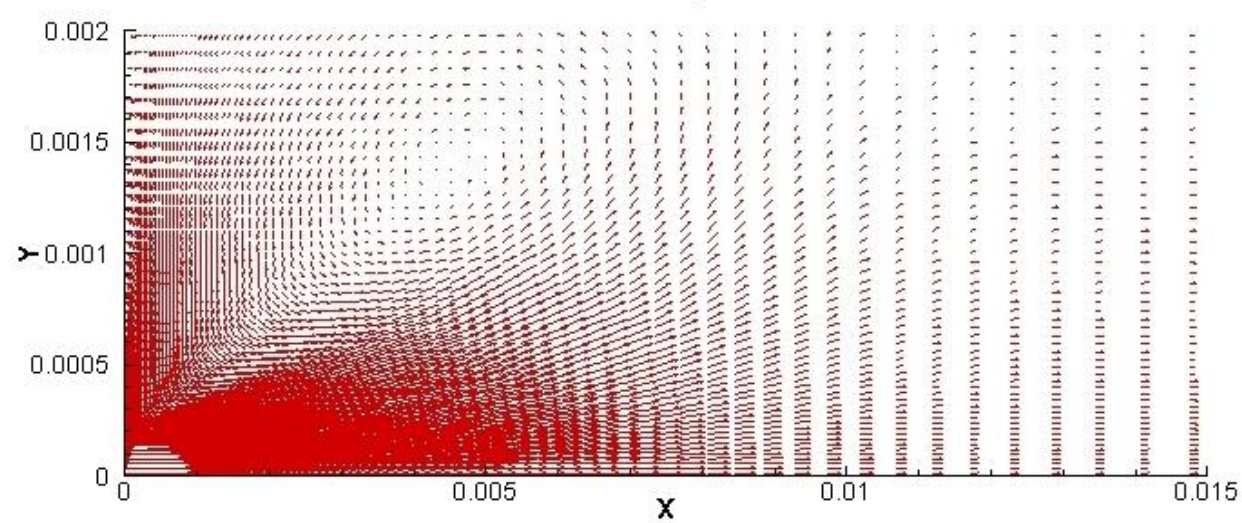

b)

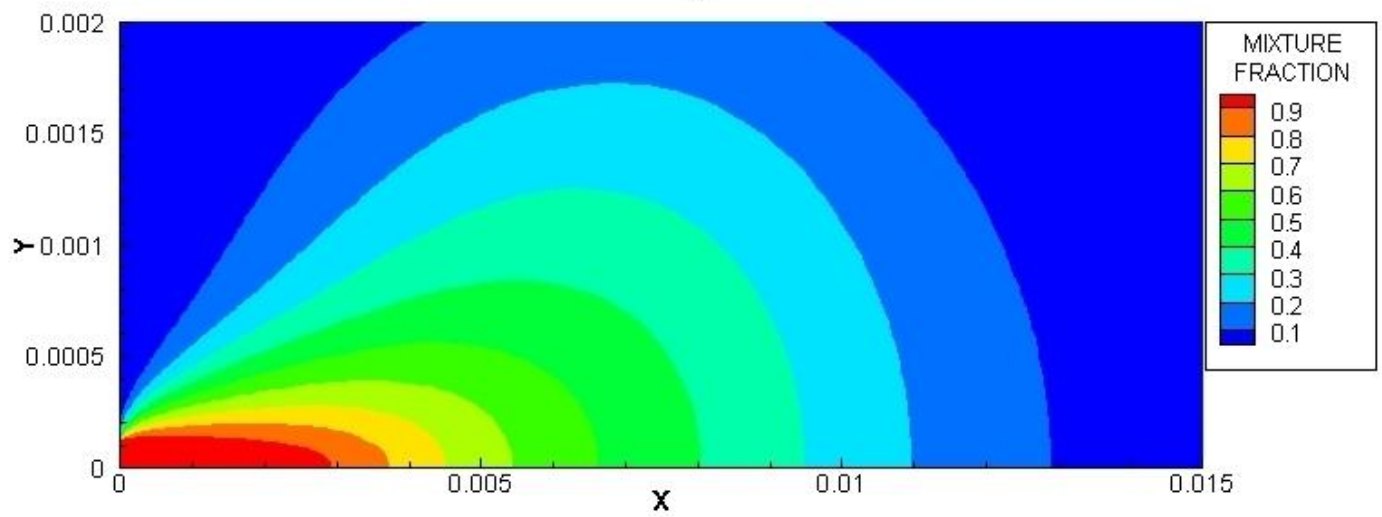

c)

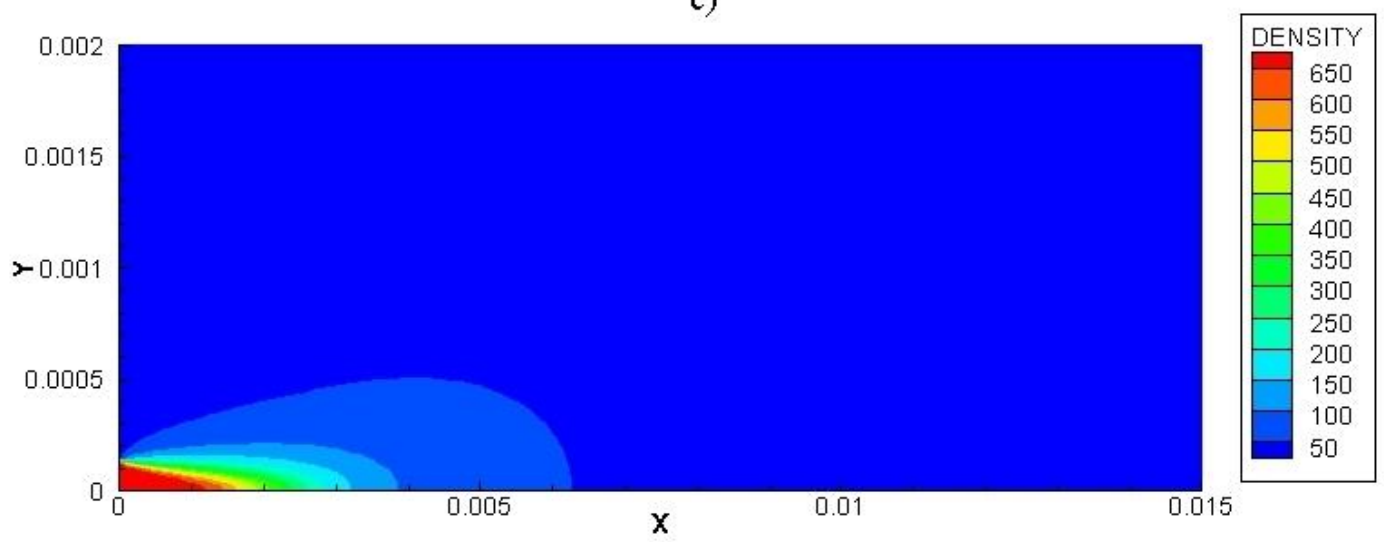

Figure 3 - Velocity and scalar fields of the jet with a density ratio of $\omega=0.035$ and a chamber pressure of $\mathrm{Pr}=0.642$, (a) Velocity vectors, (b) Mixture fraction contours, (c) Density contours. 
exposed in Ref. 18 is the jet growth rate. The jet growth rate is calculated by the tangent of the half with of half maximum of the velocity (HWHMV), this parameter is given by the calculation at each axial point of the radial distance to the centerline at which the axial velocity $(U)$ is half of the velocity at the centerline. However other parameters were used in the characterization of the jet allowing also some interesting conclusions and are shown in the present paper.

Figures 2 and 3 show the typical velocity and scalar fields of the jet for test conditions 1 (Figure 2) and 3 (Figure 3) of Table 1. In both cases the jet structure is very similar, in the velocity field is visible the appearance of the entrainment phenomena close to the injector, with the jet fluid dragging the ambient fluid. This phenomenon causes the appearance of a vortex above the jet. It appears that the position of the center of the vortex is influenced by the density ratio, when the density ratio increases the vortex center moves downstream. The mixture fraction and density scalar field show the rapid change of the jet structure at the injector exit. The density scalar field shows a faster decrease after the injector than the mixture fraction scalar field. This suggests that the density rapid decreased is not caused by the mixture of the injected fluid with the less dense ambient fluid but indeed by a rapid expansion that the injected fluid may experiences as it exits the injector.

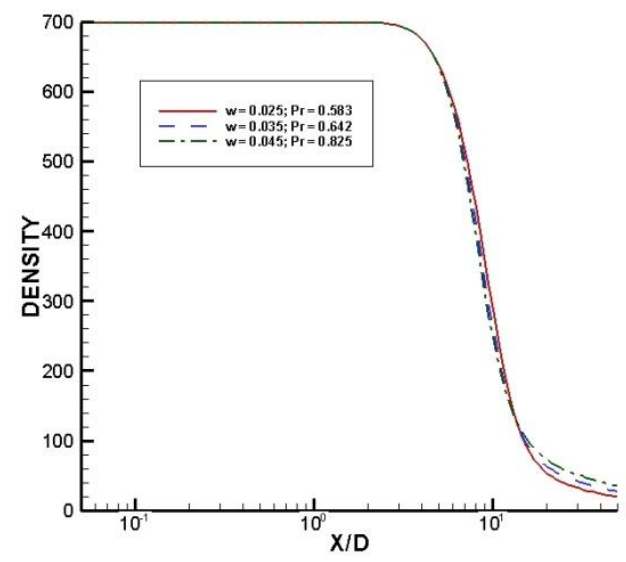

Figure 4 - Axial variation of the centerline density.

Figure 4 shows the axial variation of the centerline density in a logarithmic scale for cases 1 to 3 of Table 1 . It is visible that the centerline density has a very similar behavior for the three cases with a first stage of almost constant density followed by a stage with a fast decrease of the centerline density and then stabilization around the ambient density value. A closer look to the graphic allows however to perceive that the case with a higher ambient pressure has a slightly higher decrease rate which is in agreement with the density scalar field observed in Figures 2 and 3 . The length of the potential core based on the density is defined by the initial, almost constant, stage of the axial variation of the centerline density which lasts until an approximately length of $X / D=3$ diameters for the three studied cases.

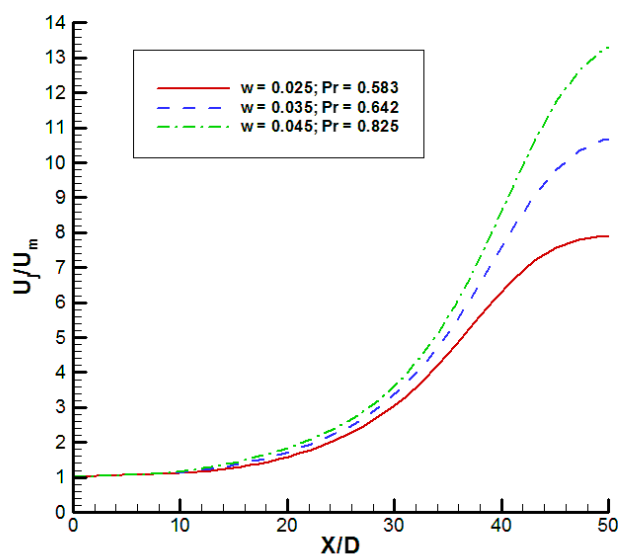

Figure 5 - Centerline velocity decay.

The centerline velocity decay is shown in Figure 5 for the cases 1, 2 and 3. The velocity decay has a similar profile for the three cases with a first stage with almost no decay followed by a stage with a higher rate of decay and finally a stabilization like observed in Figure 4 for the variation of density. A higher rate of velocity decay is observed for the cases with higher ambient pressure indicating a greater interaction between the injection and ambient fluids. The length of the potential core based on the velocity decay is defined in the present work by the axial distance to the injector at which the centerline velocity has decayed $10 \%$. The length of the potential core is between X/D = 7.9 and $X / D=7.47$ diameters respectively for cases 1 and 3 . Thus it is verified that the length of the potential core based on centerline velocity decay has a substantially different value that the approximately $X / D=$ 3 diameters found for the potential core based on the centerline density. This is an 


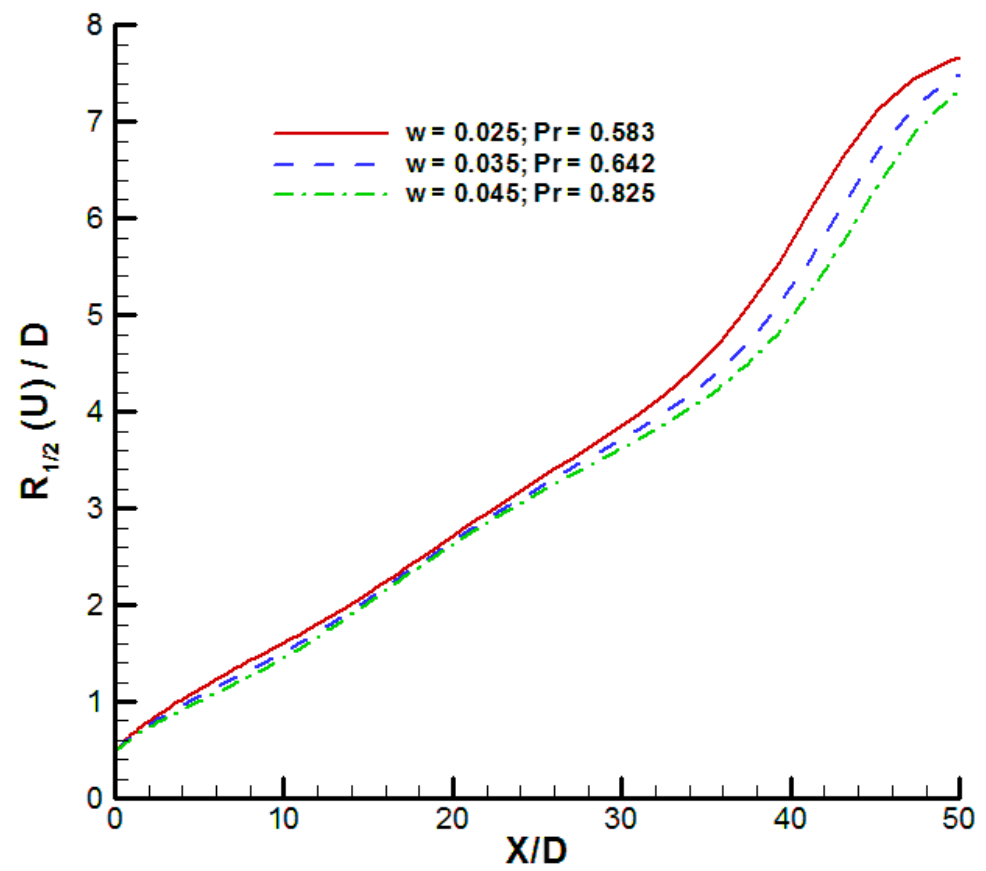

Figure 6 - Half width of half maximum of the velocity.

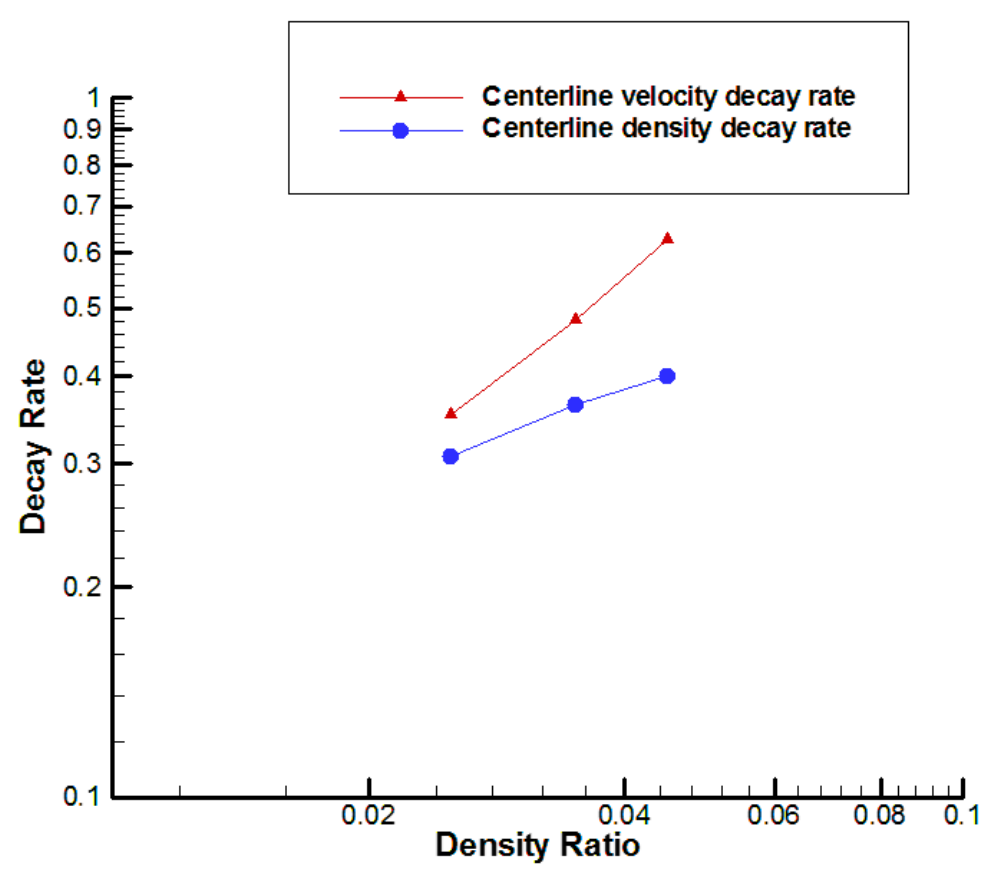

Figure 7 - Decay rate of the centerline velocity and density. 
interesting result, as in previous works the length of the potential core based on the density has approximately the same value as the one based on the velocity decay, and hypothesizes that the injected, possibly identify by the observation of the scalar fields, may contribute with kinetic energy to the jet.

Figure 6 shows the half width of the half maximum of the velocity (HWHMV) for the cases 1, 2 and 3 of Table 1. The three cases have a similar evolution of the HWHMV across the domain of study with an almost linear first stage and a second non linear stage. The tangent of the slope of the almost linear stage corresponds to the jet growing rate. Observing the graphic it is possible to conclude that the jet growing rate is very similar for the three studied cases with only a slightly decrease as the chamber pressure increases. This was however not an expected result as in several previous work the increase of the chamber pressure causes an increase in the jet growing rate.

In Figure 7 are shown the decay rates of velocity and density after the potential core for the cases of Table 1. For both velocity and density an increase in the injection chamber pressure has as result an increase of the decay rate which is in agreement with previous works from other authors, however this effect is more pronounced in the velocity decay. The velocity also shows for the three cases a higher decay rate then the density although is important to remember that the density decay starts earlier then the velocity decay due to the longer potential core obtained for the velocity.

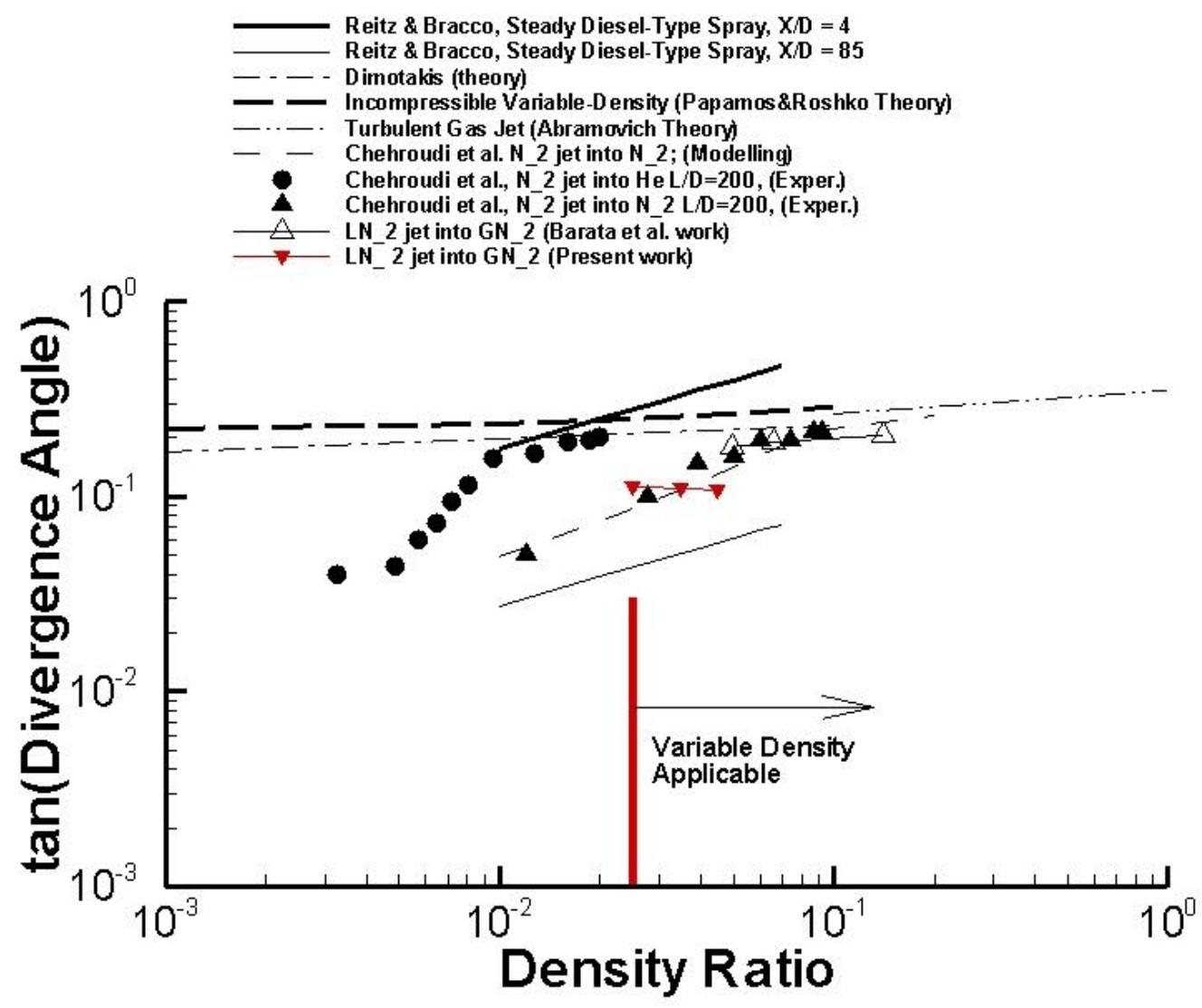

Figure 8 - Tangent of the spreading angle versus the chamber-to-injectant density ratio and representation if limit of applicability of the Variable Density approach. 
Figure 8 is similar to the graphic presented by Barata et al. ${ }^{18}$ and shows the tangent of the jet spreading angle, calculated by the half width of half maximum of the velocity, in order to the density ratio. In addition to the three cases of Table 1 the graphic contains also results obtained by other authors in previous investigations including the results of Barata et al. ${ }^{18}$, the investigation which the present work pretends to extend. The results achieved in the present work are represented in red while those achieved by other authors are represented in black. Obversing the graphic it is visible that the result obtained for a density ratio of $\omega=0.025$ agree very well with the experimental result obtained by Chehroudi et $a .^{23}$, the result obtained for a density $\omega=0.035$ is in total agreement with the modeling results of the same author. The $\omega=0.045$ density ratio case shows a slight divergence from previous result but is still very close to the modeling result of Chehroudi et al. ${ }^{23}$. The test of the present variable density approach to lower density ratios, which are not presented here, allow the conclusion that the density ratio of $\omega=$ 0.025 is the lowest value liable to be modeled by the present mathematical model. This conclusion is represented in Figure 8 by the vertical red line that marks the limit of applicability of the variable density approach.

The influence of the injection velocity in jet behavior was also subject of analysis at the present investigation. The objective was to perform a better characterization of the mathematical model. In order to achieve this objective the variable density approach was tested for different injection velocities as presented in Table 2.

Figure 9 shows the velocity and scalar fields for the test case 7 of Table 2. It is visible that the jet has the same structure found in Figure 2 corresponding to the case 4 of Table 2. It's however possible to verify that the vortex center appears in localization further downstream, also the mixture fraction and density show a slower evolution across the centerline when compared with the case with slower injection velocity. The axial variation of the centerline density shown in Figure 10 corroborates the observation stated above. The centerline density shows a slower decrease for higher injection velocities. One interesting observation is the increase of the potential core based on density as the injection velocity increases. Similar observations are made for the centerline velocity decay. As the injection velocity increases the potential core based on the velocity decay increases and the centerline velocity shows a lower rate of decay, this is somehow contrary to the expected since an increase in the injection velocity should lead to an increase of drag forces between the injected and the ambient fluid and this way increase the velocity decay rate. Like in the axial variation of the centerline also for the centerline velocity decay the case 5 with an injection velocity of $U_{\text {in }}=5 \mathrm{~m} . \mathrm{s}^{-1}$ shows a behavior different from the tendency followed by the other three cases.

The half width of half maximum of the velocity is shown in Figure 11. Again the case 4 of Table 2 shows a different HWHMV structure then the other three cases not having an almost linear stage. By the observation of the graphic can be concluded that an increase of the injection velocity causes a decrease in the jet spreading angle.

\section{Conclusions}

Barata et al. [18] had concluded that the variable density mathematical model could be successfully used in the study of cryogenic jets injected initially at subcritical temperatures into an environment at a supercritical temperature over a range of subcritical and supercritical pressures [18]. The objective of the present work was to extend the study performed by Barata et al. [18] to lower chamber-to-injection fluid density ratios. Like in Barata et al. [18], for this investigation an injection of cryogenic liquid nitrogen into a gaseous nitrogen environment was simulated.

In the analysis of the obtained result were identified some interesting new results. The fastest mixture fraction scalar field evolution when compared with the density scalar field together with the difference between the potential core based on density and the one based on velocity decay, suggests that a vigorous expansion of the injected fluid happens as it enters the injection chamber. And the longer potential core based on the velocity decay indicated that expansion may somehow contribute with kinetic energy to the jet.

The results of the half with of half maximum of the velocity allowed the determination of the jet growth rate with are in general agreement with the results obtained by Chehroudi et al. ${ }^{23}$ described in Ref. 18. By the analysis of the results of the HWHMV obtained to density ratios lower than 
$\omega=0.025$, it was concluded that below this value no more agreement was found with previous experimental and numerical results. This establishes the limit of applicability of the variable density approach to cryogenic liquid jet into a supercritical temperature and subcritical pressure ambient.

\section{References}

${ }^{1}$ Chehroudi, B., Cohn, R., and Talley, D., "Visual Characteristics and Initial Growth Rates of Round Cryogenic Jets at Subcritical and Supercritical Pressures," Physics of Fluids, Vol. 14, No. 2, 2002, pp. 850-861.

${ }^{2}$ Bellan, J., "Supercritical (and Subcritical) Fluid Behavior and Modeling: Drops, Streams, Shear and Mixing Layers, Jets and Sprays," Progress in Energy and Combustion Science, Vol. 26, Issues 4-6, 2000, pp. 329366

${ }^{3}$ Yang, V., "Modeling of Supercritical Vaporization Mixing, and Combustion Processes in Liquid-Fueled Propulsion Systems," Proceedings of the Combustion Institute, Vol. 28, 2000, pp. 925-942.

${ }^{4}$ Segal, C., and Polikhov, S.A., "Subcritical to Supercritical Mixing," Physics of Fluids, Vol. 20, Issue 5 2008, pp. 052101-1-052101-7.

${ }^{5}$ Oschwald, M., Smith, J. J., Branam, R., Hussong, J., Schik, A., Chehroudi, B. and Talley, D. (2006) "INJECTION OF FLUIDS INTO SUPERCRITICAL ENVIRONMENTS", Combustion Science and Technology, 178: $1,49-100$.

${ }^{6}$ Mayer, W.O.H., Schick, A. H. A., Vielle, B., Chauveau, C., Gökalp, I., Talley, D.G., and Woodward, R.D., "Atomization and Breakup of Cryogenic Propellants under High-Pressure Subcritical and Supercritical Conditions," Journal of Propulsion and Power, Vol.14, No.5, 1998, pp.835-842.

${ }^{7}$ Chehroudi, B., Cohn, R., and Talley, D., "Cryogenic Shear Layers: Experiments and Phenomenological Modeling of the Initial Growth Rate under Subcritical and Supercritical Conditions," International Journal of Heat and Fluid Flow, Vol. 23, Issue 5, 2002, pp. 554-563.

${ }^{8}$ Givler, S.D., John Abraham, J., "Supercritical Droplet Vaporization and Combustion Studies," Progress in Energy and Combustion Science, Vol. 22, Issue 1 1996, pp. l-28.

'Zhang, H., Raghavan, V., Gogos, G., "Subcritical and Supercritical Droplet Evaporation within a ZeroGravity Environment: Low Weber Number Relative Motion," International Communications in Heat and Mass Transfer, Vol. 35, Issue 4, 2008, pp. 385-394.

${ }^{10}$ Nomura, H., Ujiie, Y., Rath, H.J., Sato, J., Kono, M., "Experimental Study of High-Pressure Droplet Evaporation using Microgravity Conditions," TwentySixth Symposium (International) on Combustion/The Combustion Institute, 1996, pp. 1267-1273.
${ }^{11}$ Tsue, M., Nomur, H., Niwa, M., Miyano, H., Sato, J., Kono, M., "Evaporation of a Fuel Droplet in a High Pressure High Temperature Atmosphere," Japan Society for Aeronautical and Space Sciences, Journal (ISSN 00214663), Vol. 37, No. 420, 1989, pp. 21-28.

${ }^{12}$ Fieberg, C., Reichelt, L., Martin, D., Renz, U., and Kneer, R., "Experimental and Numerical Investigation of Droplet Evaporation under Diesel Engines Conditions," International Journal of Heat and Mass Transfer, Vol. 52, Issues 15-16, 2009, pp. 3738-3746.

${ }^{13}$ Zhu, G.S., and Aggarwal, S.K., "Transient Supercritical Droplet Evaporation with emphasis on the Effects of Equation of State", International Journal of Heat and Mass Transfer, Vol. 43, Issue 7, 2000, pp. 1157-1171.

${ }^{14}$ Emekwuru, N.G., Watkins, P., "Analysis of a TwoFluid Sprayer and its Use to Develop the Number Size Distribution Moments Spray Model Part II: Computational Analysis," Atomization and Sprays, Vol. 20, Issue 8, 2010, pp. 653-672.

${ }^{15}$ Oschwald, M., and Schik, A., "Supercritical Nitrogen Free Jet Investigated by Spontaneous Raman Scattering," Experiments in Fluids, Vol. 27, No. 6, 1999, pp. 497-506.

${ }^{16}$ Mayer, W., and Telaar, J., "Investigation of Breakup of Turbulent Cryogenic Variable-Density Jets," Atomization and Sprays, Vol. 12, Issue 5-6, 2002, pp. 651-666.

${ }^{17}$ Sanders, J. P. H., B. Sarh, B., Gokalp, I., “Variable Density Effects in Axisymmetric Isothermal Turbulent jets: a Comparison Between a First- and a Second-order Turbulence Model," Int. J. Heat Mass Transfer. Vol. 40, No. 4, 1997, pp. 823-842.

${ }^{18}$ Barata, J.M.M., Gokalp, I., Silva, A.R.R., "Numerical Study of Cryogenic Jets under Supercritical Conditions," Journal of Propulsion and Power, Vol. 19, No.1, 2003, pp. 142-147.

${ }^{19}$ Zong, N., Meng, H., Hsieh, S., and Yang, V., "A Numerical Study of Cryogenic Fluid Injection and Mixing under Supercritical Conditions," Physics of Fluids, Vol. 16 , No. 12,2004 , pp. 4248-4261.

${ }^{20}$ Sierra-Pallares, J., Parra-Santos, M.T., GarcíaSerna, J., Castro, F., Cocero, M.J., "Numerical Analysis of High-pressure Fluid Jets: Application to RTD Prediction in Supercritical Reactors", Journal of Supercritical Fluids, Vol. 49, 2009, pp. 249-255.

${ }^{21}$ Schmitt, T., Selle, L., Cuenot, B., and Poinsot, T., "Large-Eddy Simulation of Transcritical Flows", Comptes Rendus Mécanique, Vol. 337, Issues 6-7, June-July 2009, pp. 528-538.

${ }^{22}$ Kim, T., Kim, Y., Kim, S., "Numerical study of cryogenic liquid nitrogen jets at supercritical pressures," The Journal of Supercritical Fluids, Vol. 56, Issue 2, 2011, pp. 152-163.

${ }^{23}$ Chehroudi, B., Cohn, R., and Talley, D., "Spray/Gas Behaviour of Cryogenic Fluids under Suband Supercritical Conditions", Eighth International Conference on Liquid Atomization \& Sprays Systems, ICLASS-2000, Pasadena, California, USA, July 16-20, 2000. 

Attachment 3

Paper send to AIAA 



\title{
EVALUATION OF NUMERICAL VARIABLE DENSITY APPROACH TO CRYOGENIC JETS
}

\author{
E.L.S.F. Antunes ${ }^{1}$, A. R. R. Silva ${ }^{2}$, J. M. M. Barata ${ }^{3}$ \\ Universidade da Beira Interior, 6200-001 Covilhã, Portugal
}

\begin{abstract}
The present work is devoted to study cryogenic nitrogen jets in high subcritical conditions. Fuel injection is one of the great challenges in engineering of diesel engines, gas turbines and rocket engines, combining in the last one also the injection of oxidizer. It is widely known that the increase of operation pressures and temperatures increases engine efficiency and reduces fuel specific consumption. Thus, it is a general trend in modern engines the operation in increasingly higher pressures. However at higher chamber pressures the injected fluids may experience ambient conditions exceeding the critical values. Several authors stated that at these conditions the injected fluids suffers a change of its properties, and the traditional two-phase flow models cannot correctly predict the jet behavior at these conditions, thus new computational models are needed for these specific conditions. Barata et al. [18] performed a numerical investigation aimed to evaluate the applicability of an incompressible but variable density model in liquid jets under sub-to-supercritical conditions. The results achieved agree well with the experimental data but they only considered intermediate density ratios from 0.05 to 0.14 . The objective of the present work was to extend the investigation of Ref. 18 to lower density ratios from 0.025 to 0.045 which correspond to cases with subcritical chamber pressures. The obtained results agree well with the experimental and numerical data of Chehroudi $e t$ al. presented in Ref. 18. It was also found in this work that the computational model does not offers valid results for density ratios.
\end{abstract}

\section{Nomenclature}

$B \quad=$ transfer number

$\beta_{v} \quad=$ evaporation rate

$C_{\mu} \quad=$ coefficient in turbulence model

$d_{0} \quad=$ initial droplet diameter

$d \quad=$ droplet diameter

$D \quad=$ injector diameter $[\mathrm{m}]$, normalized droplet diameter $\left(\mathrm{d}(\mathrm{t}) / \mathrm{d}_{0}\right)$

$\varepsilon=$ dissipation rate of turbulent energy

$f \quad=$ mixture fraction

$F \quad=$ mean mixture fraction

$i \quad=$ axial direction index

$j \quad=$ radial direction index

$k \quad=$ turbulent kinetic energy

$\phi \quad=$ generalized variable

$\omega=$ chamber-to-injection fluid density ratio $\left(\rho_{\infty} / \rho_{0}\right)$

$P_{c r} \quad=$ critical pressure $[\mathrm{MPa}]$

$P_{\infty} \quad=$ chamber ambient pressure $[\mathrm{MPa}]$

$P_{r} \quad=$ reduced pressure $\left(\mathrm{P}_{\infty} / \mathrm{P}_{\mathrm{cr}}\right)$

$\rho \quad=$ density $\left[\mathrm{kg} \cdot \mathrm{m}^{-3}\right]$

$\rho_{0} \quad=$ injected fluid density $\left[\mathrm{kg} \cdot \mathrm{m}^{-3}\right]$

$\rho_{\infty} \quad=$ injection chamber's fluid density [kg. $\mathrm{m}^{-3}$ ] $=$ radial coordinate $[\mathrm{m}]$

\footnotetext{
${ }^{1}$ MEng Student, Aerospace Sciences Department, Rua Marques Avila e Bolama.

${ }^{2}$ Assistant Professor, Aerospace Sciences

Department, AIAA Member.

${ }^{3}$ Full Professor, Aerospace Sciences Department, AIAA Associate Fellow.
}

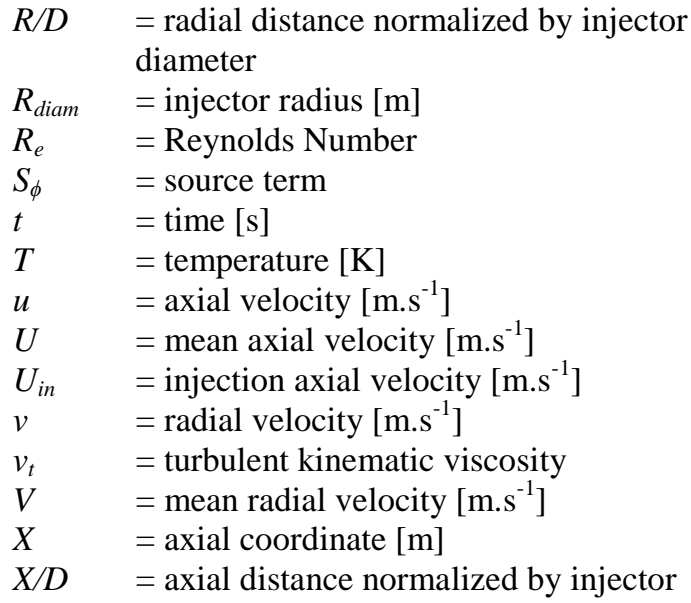

diameter

\section{$\underline{\text { Introduction }}$}

Fuel injection presents itself as one of the great challenges in engineering of diesel engines, gas turbines and rocket engines, combining in the last one also the injection of oxidizer. It is widely known that the increase of operating pressure and temperatures in combustion chamber, or thrust chamber in rocket engines, leads to an increase of engine efficiency, reducing this way the fuel consumption. Thereby is a general trend in new engine designs to operate with higher chamber pressures and temperatures. Also the appearance of new and more resistant materials is other reason that could make grow this tendency. As a result of these increasingly higher pressures, the injected fluids may experience ambient conditions 
exceeding the critical values. The Space Shuttle Main Engine and the Vulcain with thrust chamber pressures of respectively $22.3 \mathrm{MPa}$ and $28.2 \mathrm{MPa}$ are both examples of engines in which the chamber pressure exceeds the critical pressure of $\mathrm{P}_{c r}=5.043$ $\mathrm{MPa}$ for liquid oxygen and $\mathrm{P}_{\mathrm{cr}}=1.28 \mathrm{MPa}$ for liquid hydrogen ${ }^{1}$. In these application, the initial temperature of the oxygen can initially be below the critical temperature of the oxygen $\left(\mathrm{T}_{\mathrm{cr}}=154.58\right.$ $\mathrm{K})$ but it then undergoes to a transition, when in the combustion chamber, reaching supercritical temperatures. At these conditions the liquid fuel is on supercritical conditions and its physical state is named as fluid. As the fluid reach pressure and temperature values exceeding the critical ones, it suffers significantly changes in its properties. The effective mass diffusivity, the surface tension and the latent heat of the liquid all vanish in critical conditions. On the other hand, the heat capacity at constant pressure, $C \mathrm{p}$, the isentropic compressibility, $\kappa_{\mathrm{s}}$, and the thermal conductivity, $\lambda$, all become infinite [2]. These changes in the fluid behavior cause the inapplicability of the traditional two-phase flow models used in fuel injection under subcritical conditions, thus there is a need to develop new models with can correctly be applied to supercritical fuel injection.

Several authors investigated the fuel injection in supercritical condition both in experimentally and numerically ${ }^{3-22}$. The first experimental investigations performed used techniques like photography and shadowgraphy, and had as principal objective the study of the visual structure of the jet without obtaining any quantitative result $^{1,6}$, these investigations observed that the jet structure suffers significantly changes as the pressure increases, firstly the surface tension reduction leads to the formations of jet ligaments and droplets that detach from the main jet structure and a further increase of the chamber pressure into supercritical conditions leads to gas-gas like structure of the jet. In more recent experimental works, along with qualitative characterization, were made quantitative studies in which results like the jet spreading angle, density and temperature were obtained ${ }^{4,7,15,16}$. These quantitative experimental results allowed the comparison with the results obtained in numerical studies and this way validate the numerical models ${ }^{17-22}$.

Barata et al. ${ }^{18}$ performed an initial investigation aimed to evaluate the capabilities of a computational method developed for incompressible but variable density flows when applied to supercritical conditions. Their results have show a good agreement with the experimental data, but they only considered intermediate density ratios from 0.05 to 0.14 . The present work extends the investigation of Ref. 18 to lower density ratios, corresponding to sub/near- critical conditions, and investigates the limits of application of the variable density approach to supercritical jets. An injection velocity study was also performed in the present work with intend of better characterize the variable density approach.

Mathematical Model

\section{Governing Equations}

The method to solve variable density jet flows is based on the solution of the conservation equations for momentum and mass. Turbulence is modeled with the " $k \sim \varepsilon$ " turbulence model. A similar method has been used for three-dimensional or axisymmetric flows and only the main features are summarized here.

In the conservation equations, mass weighted averaging is applied to avoid the appearance of many terms involving density fluctuations for which additional models are needed. A mass averaged quantity is defined as

$$
\tilde{\phi}=\frac{\overline{\rho \phi}}{\bar{\rho}} \text {. }
$$

For the governing equations the standard parabolic truncation is employed. The mass averaged partial differential equations governing the steady, variable density axisymmetric flow may be written in cylindrical polar coordinates as

$$
\begin{gathered}
\frac{\partial \bar{\rho} U U}{\partial x}+\frac{1}{r} \frac{\partial r \bar{\rho} U V}{\partial r}=-\frac{\partial \bar{p}}{\partial x}-\frac{1}{r} \frac{\partial r \bar{\rho} \frac{\tilde{u^{\prime} v^{\prime}}}{\partial r}}{\partial \bar{\rho} U V} \\
\frac{\partial x}{\partial} \frac{1}{r} \frac{\partial r \bar{\rho} V V}{\partial r}=-\frac{\partial \bar{p}}{\partial x}-\frac{1}{r} \frac{\partial r \bar{\rho} \frac{\tilde{v^{\prime} v^{\prime}}}{\partial r}+\bar{\rho} \frac{\tilde{w^{\prime} w^{\prime}}}{r}}{}
\end{gathered}
$$

and the continuity equation as

$$
\frac{\partial \bar{\rho} U}{\partial x}+\frac{1}{r} \frac{\partial r \bar{\rho} V}{\partial r}=0
$$

To describe mixing of gases, the mixture fraction $F$, that represents the mass fraction of the nozzle fluid, is introduced. It obeys a convectiondiffusion equation of the form

$$
\frac{\partial \bar{\rho} U F}{\partial x}+\frac{1}{r} \frac{\partial r \bar{\rho} V F}{\partial r}=-\frac{1}{r} \frac{\partial r \bar{\rho} \frac{\tilde{v^{\prime} f^{\prime}}}{\partial r}}{\partial r}
$$


In " $\mathrm{k} \sim \varepsilon$ " turbulence model, the Reynolds stresses are expressed in terms of the local strain rate:

$-\bar{\rho} \overline{u_{i}^{\prime}{ }_{i}^{\prime}}{ }_{j}=\bar{\rho}\left(v_{t}+v\right)\left(\frac{\partial \tilde{u}_{i}}{\partial x_{j}}+\frac{\partial \tilde{u}_{j}}{\partial x_{i}}\right)-\frac{2}{3} \delta_{i j}\left(\bar{\rho} k+\bar{\rho}\left(v_{t}+v\right) \frac{\partial \tilde{u}_{j}}{\partial x_{j}}\right)$

with

$$
v_{t}=C_{\mu} \frac{k^{2}}{\varepsilon}
$$

The scalar flux in equation (5) is approximated with a gradient transport assumption

$$
\overline{u_{i}^{\prime} f^{\prime}}=-\frac{v_{t}}{\sigma_{f}} \frac{\partial F}{\partial x_{i}}
$$

From the foregoing we can deduced the parabolized set of equations in cylindrical coordinates where the generalized equation is

$$
\frac{\partial}{\partial x}(\bar{\rho} U \tilde{\phi})+\frac{1}{r} \frac{\partial}{\partial r}(r \bar{\rho} \tilde{\phi})=\frac{1}{r} \frac{\partial}{\partial r}\left(r \bar{\rho} D \frac{\partial \tilde{\phi}}{d r}\right)+S_{\phi}
$$

where $\tilde{\phi}$ may stand for any of the velocities, turbulent kinetic energy, dissipation, or scalar property, and $S_{\phi}$ take on different values for each particular $\tilde{\phi}$, described in detail by Sanders et $\mathrm{al}^{17}$.

The mean density can be obtained from the mean mixture fraction using the equation state. With constant pressure this leads to

$$
\frac{1}{\bar{\rho}}=\frac{F}{\rho_{o}}+\frac{1-F}{\rho_{\infty}}
$$

where density fluctuations have been neglected. This is allowed in isothermal jets because the instantaneous density, for which equation (10) is exact, is approximately a linear function of the instantaneous mixture fraction ${ }^{18}$.

\section{Numerical Method}

The governing equations are solved using a parabolized marching algorithm which resembles the (elliptic) TEACH code [17]. The computations are performed by using the continuity equation to obtain the radial velocity $(V)$. Using the radial momentum equation for $V$ and solving a pressure correction equation for $V$ in radial direction did not show any difference with the use of the continuity equation [17]. In this approach the numerical model was applied to variable density jets and for the present case it was used for the study of liquid cryogenic jets under

In order to determine the tangent of the jet spreading angle is used the Half Width of Half Maximum of the Velocity (HWHMV), this routine has the function of determine the radial distance from the centerline $(r)$ at which the axial mean velocity $(U)$ is half of the maximum velocity localized in the centerline. This routine analysis all the points in the radial direction comparing them with half of the centerline velocity, when the routine finds a point bellow this value it saves the point before that.

\section{$\underline{\text { Boundary Conditions }}$}

There are four boundaries in the computational domain, in these boundaries dependent variables are specified: an inlet and outlet plane, a symmetry axis and a free boundary parallel to the axis. The sensitivity of the solutions to the locations of the boundaries was investigated in previous works, and their final position is sufficiently far away from the jet so that the influence on the computed results is negligible [18]. At the inlet boundary the $1 / 7$ th power law turbulent velocity profile was used for the axial velocity at the jet exit.

$$
U=U_{\text {in }} \cdot\left(1-\frac{r}{R_{\text {diam }}}\right)^{\frac{1}{7}}
$$

The radial velocity is zero at the jet exit and in ambient. The mixture fraction is one at the jet exit and zero in the ambient. On the symmetry axis, the normal velocity vanishes, and the normal derivatives of the other variables are zero. At the outflow boundary, the gradients of dependent variables in the axial direction are set to zero.

The flow configuration can be observed in Figure 1. The injector nozzle has a diameter of $2.54 \times 10^{-4} \mathrm{~m}$ while the domain of study has an axial length of $1.77 \times 10^{-2} \mathrm{~m}$ and a radial length of $3.49 \times 10^{-3} \mathrm{~m}$. The test conditions for the present study of different densities ratios are presented in Table 1

It was also performed a parallel study with the objective of evaluate the numerical model for different injection velocities, the test conditions used are summarized in Table 2.

The mathematical program underwent small changes from its original form in order to avoid the flow reversion verified next to the outlet when at high injection velocities. 
Table 5. Summary of test conditions

\begin{tabular}{|c|c|c|c|c|c|c|c|}
\hline Case & $\mathbf{P r}$ & $\mathbf{U}_{\text {in }}[\mathbf{m} / \mathbf{s}]$ & $\mathbf{R e}$ & $\mathbf{T}_{\mathbf{0}}[\mathbf{K}]$ & $\begin{array}{c}\mathbf{\rho}_{\mathbf{0}} \\
{\left[\mathbf{K g} / \mathbf{m}^{3}\right]}\end{array}$ & $\begin{array}{c}\boldsymbol{\rho}_{\infty} \\
{\left[\mathbf{K g} / \mathbf{m}^{3}\right]}\end{array}$ & $\boldsymbol{\omega}$ \\
\hline 1 & 0,583 & 3.0 & 72060 & 100 & 700 & 17,5 & 0,025 \\
\hline 2 & 0,642 & 3.0 & 72060 & 100 & 700 & 24,5 & 0,035 \\
\hline 3 & 0,825 & 3.0 & 72060 & 100 & 700 & 31,5 & 0,045 \\
\hline
\end{tabular}

Table 6. Summary of test conditions for the injection velocity study

\begin{tabular}{|c|c|c|c|c|c|c|c|}
\hline Case & $\mathbf{P r}$ & $\mathbf{U}_{\text {in }}[\mathbf{m} / \mathbf{s}]$ & $\mathbf{R e}$ & $\mathbf{T}_{\mathbf{0}}[\mathbf{K}]$ & $\begin{array}{c}\boldsymbol{\rho}_{\mathbf{0}} \\
{\left[\mathbf{K g} / \mathbf{m}^{\mathbf{3}}\right]}\end{array}$ & $\begin{array}{c}\boldsymbol{\rho}_{\infty} \\
{\left[\mathbf{K g} / \mathbf{m}^{\mathbf{3}}\right]}\end{array}$ & $\boldsymbol{\omega}$ \\
\hline 4 & 0,583 & 3.0 & 72060 & 100 & 700 & 17,5 & 0,025 \\
\hline 5 & 0,583 & 5.0 & 120100 & 100 & 700 & 17,5 & 0,025 \\
\hline 6 & 0,583 & 10.0 & 240200 & 100 & 700 & 17,5 & 0,025 \\
\hline 7 & 0,583 & 20.0 & 480400 & 100 & 700 & 17,5 & 0,025 \\
\hline
\end{tabular}

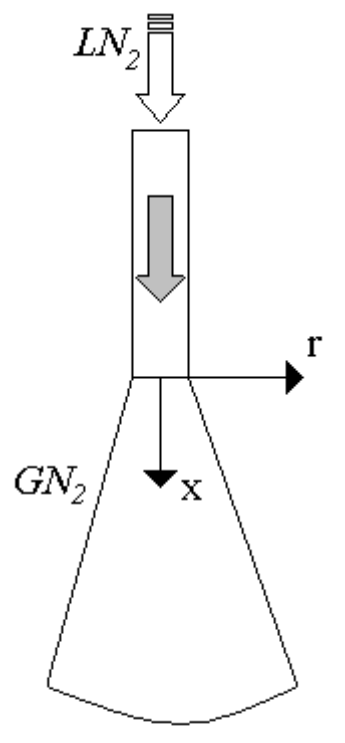

Figure 1 - Flow Configuration

\section{$\underline{\text { Results and discussion }}$}

In this section are presented the numerical results obtained at the present work and compared with the results exposed by Barata et al. ${ }^{18}$. The main objective is to extend the analysis performed by Barata et al. ${ }^{18}$ to lower density ratios and then compare them with the experimental data in order to investigate the applicability of the variable density approach to liquid jet injection into a gaseous ambient under subcritical chamber pressures but supercritical temperatures. The parameter used to compare the numerical results obtained in the present work with the results exposed in Ref. 18 is the jet growth rate. The jet growth rate is calculated by the tangent of the half with of half maximum of the velocity (HWHMV), this parameter is given by the calculation at each axial point of the radial distance to the centerline at which the axial velocity (U) is half of the velocity at the centerline. However other parameters were used in the characterization of the jet allowing also some interesting conclusions and are shown in the present paper.

Figures 2 and 3 show the typical velocity and scalar fields of the jet for test conditions 1 (Figure 2 ) and 3 (Figure 3 ) of Table 1 . In both cases the jet structure is very similar, in the velocity field is visible the appearance of the entrainment phenomena close to the injector, with the jet fluid dragging the ambient fluid. This phenomenon causes the appearance of a vortex above the jet. It appears that the position of the center of the vortex is influenced by the density ratio, when the density ratio increases the vortex center moves downstream. The mixture fraction and density scalar field show the rapid change of the jet structure at the injector exit. The density scalar field shows a faster decrease after the injector than the mixture fraction scalar field. This suggests that 
a)

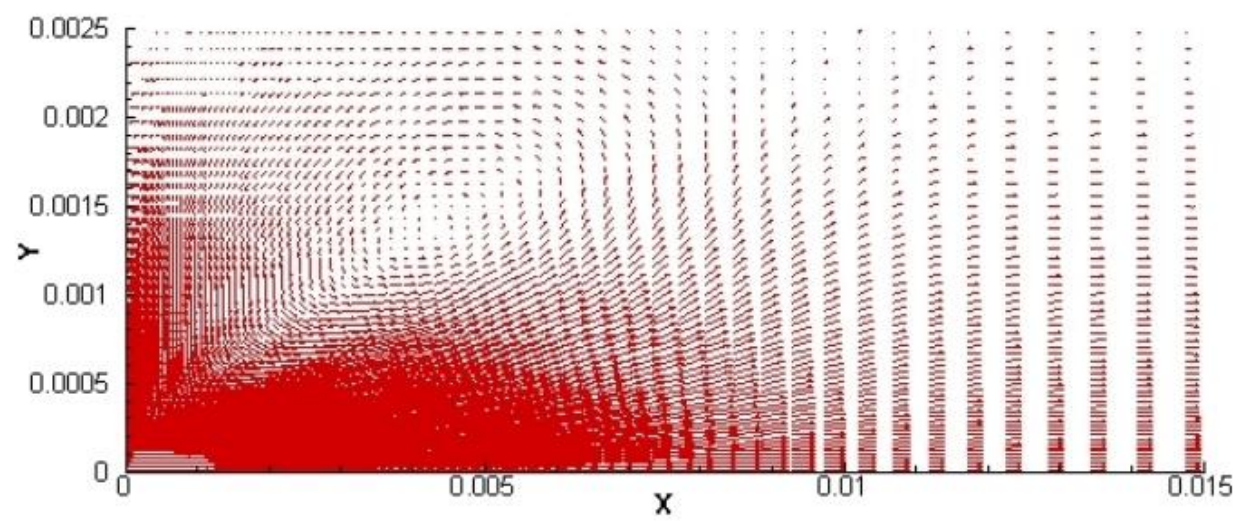

b)

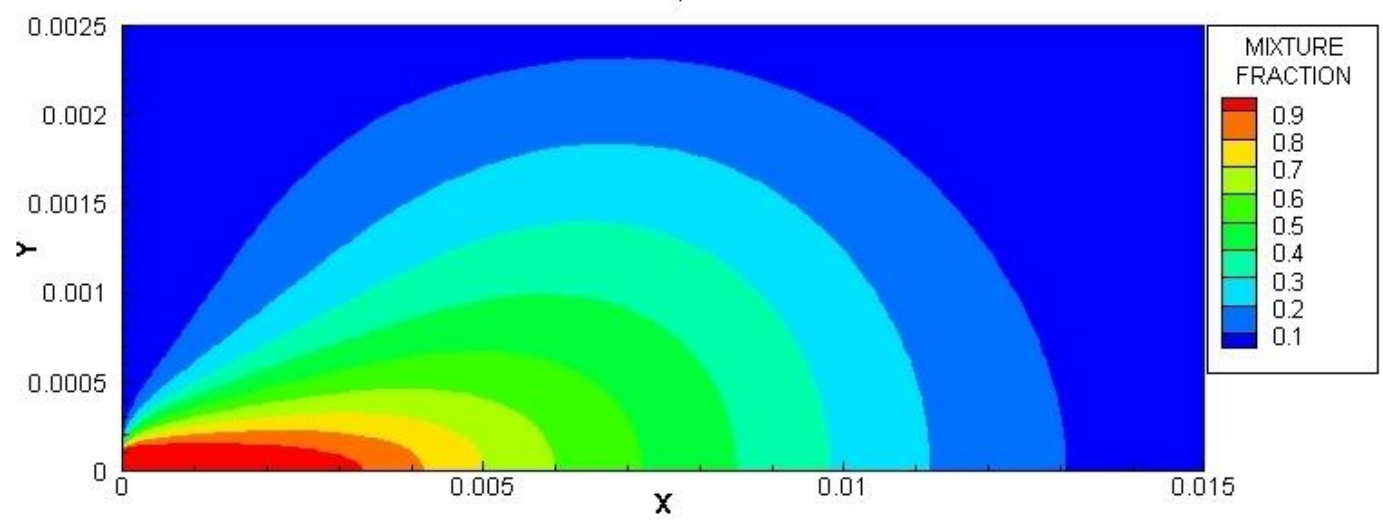

c)

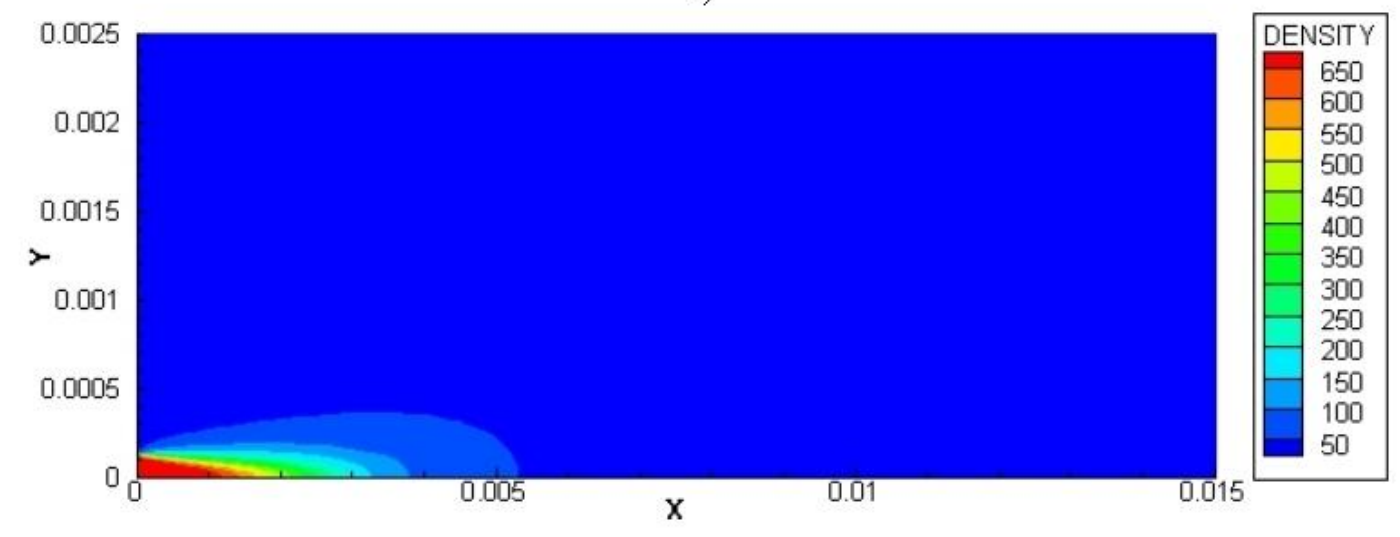

Figure 2 - Velocity and scalar fields of the jet with a density ratio of $\omega=0.025$ and a chamber pressure of $\operatorname{Pr}=\mathbf{0 . 5 8 3}$, (a) Velocity vectors, (b) Mixture fraction contours, (c) Density contours. 
a)

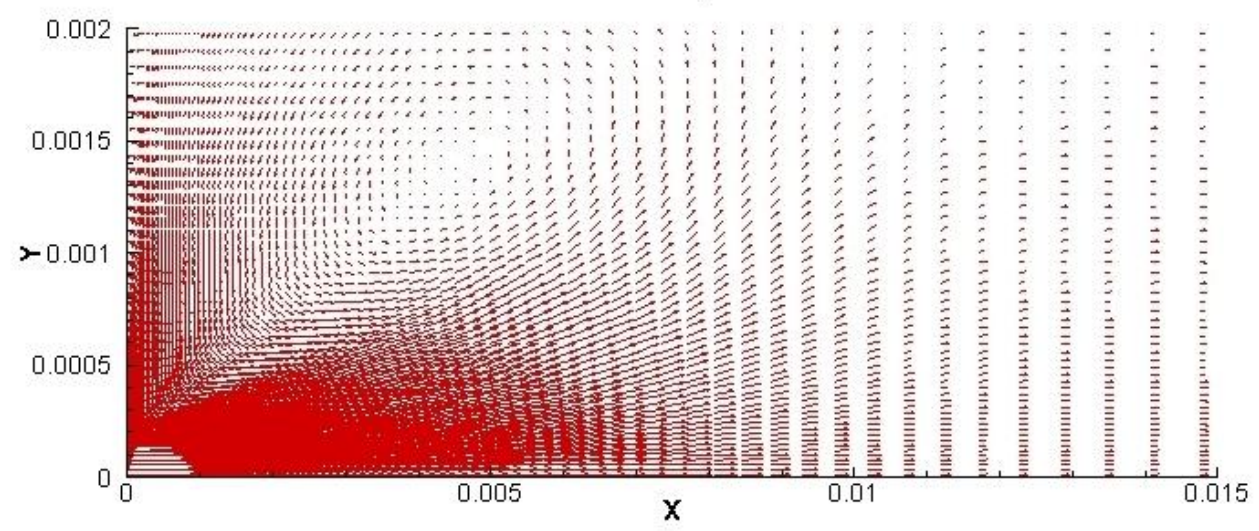

b)

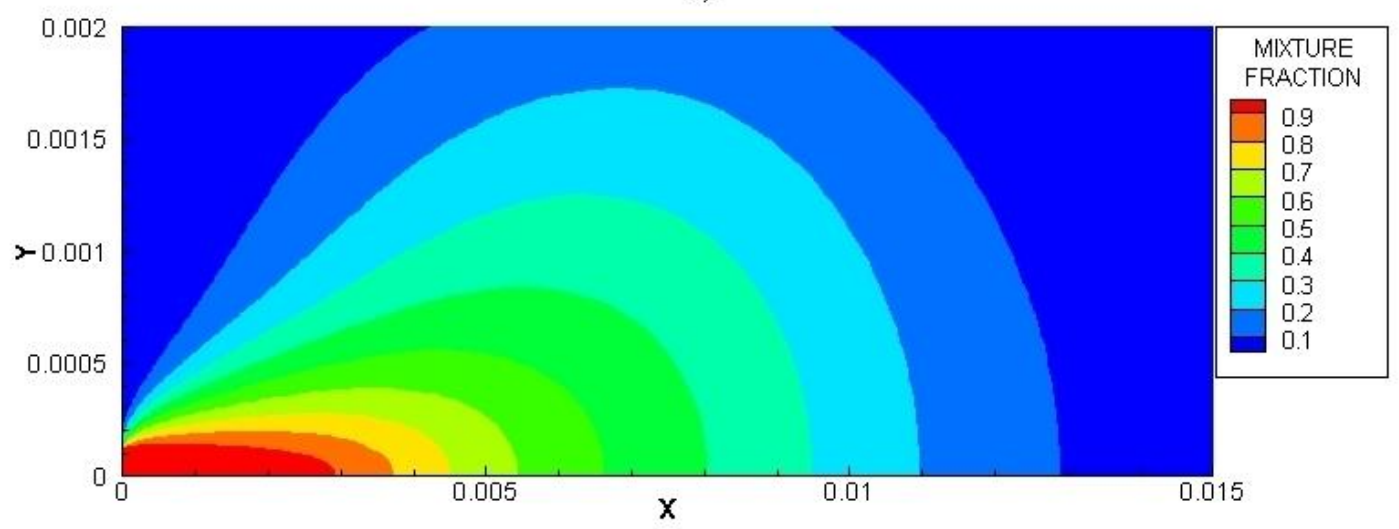

c)

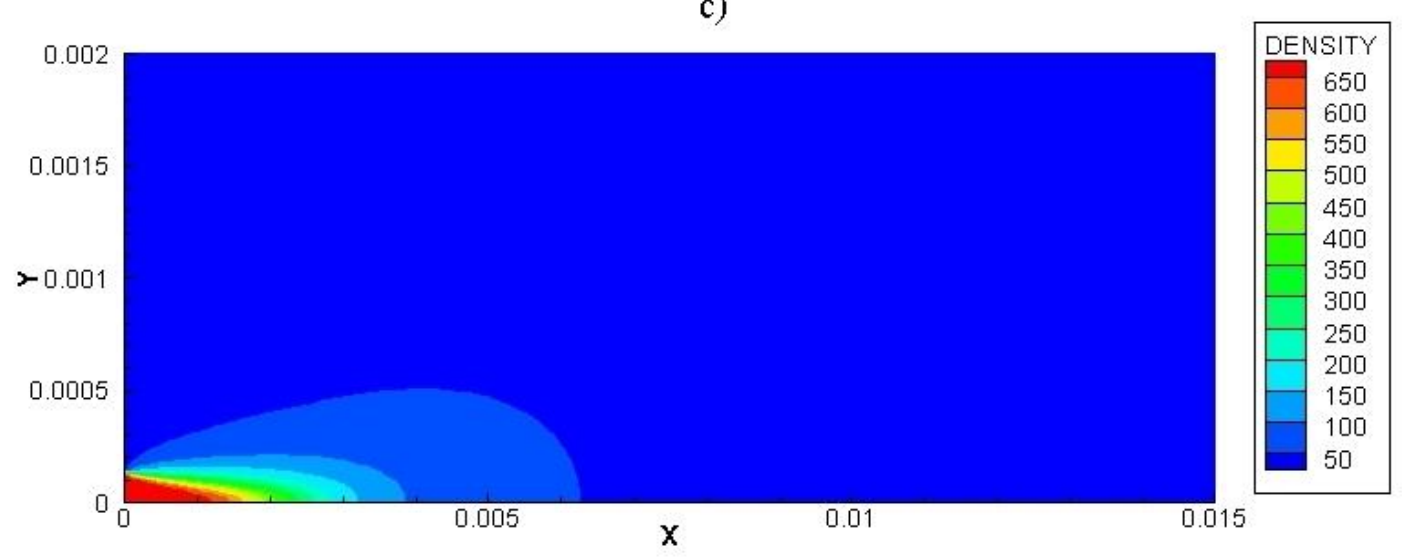

Figure 3 - Velocity and scalar fields of the jet with a density ratio of $\omega=0.035$ and a chamber pressure of $\operatorname{Pr}=\mathbf{0 . 6 4 2}$, (a) Velocity vectors, (b) Mixture fraction contours, (c) Density contours. 
ambient fluid but indeed by a rapid expansion that the injected fluid may experiences as it exits the injector.

Figure 4 shows the axial variation of the centerline density in a logarithmic scale for cases 1 to 3 of Table 1 . It is visible that the centerline density has a very similar behavior for the three cases with a first stage of almost constant density followed by a stage with a fast decrease of the centerline density and then stabilization around the ambient density value. A closer look to the graphic allows however to perceive that the case with a higher ambient pressure has a slightly higher decrease rate which is in agreement with the density scalar field observed in Figures 2 and 3 . The length of the potential core based on the density is defined by the initial, almost constant, stage of the axial variation of the centerline density which lasts until an approximately length of $\mathrm{X} / \mathrm{D}=$ 3 diameters for the three studied cases.

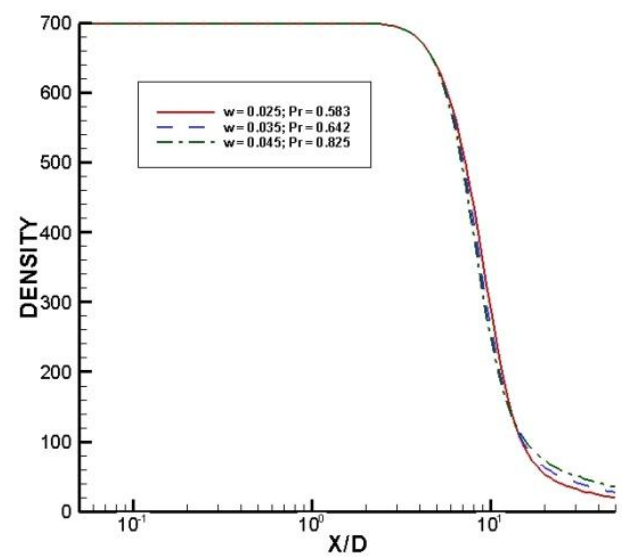

Figure 4 - Axial variation of the centerline
density.

The centerline velocity decay is shown in Figure 5 for the cases 1,2 and 3. The velocity decay has a similar profile for the three cases with a first stage with almost no decay followed by a stage with a higher rate of decay and finally a stabilization like observed in Figure 4 for the variation of density. A higher rate of velocity decay is observed for the cases with higher ambient pressure indicating a greater interaction between the injection and ambient fluids. The length of the potential core based on the velocity decay is defined in the present work by the axial distance to the injector at which the centerline velocity has decayed $10 \%$. The length of the potential core is between $\mathrm{X} / \mathrm{D}=7.9$ and $\mathrm{X} / \mathrm{D}=$
7.47 diameters respectively for cases 1 and 3. Thus it is verified that the length of the potential core based on centerline velocity decay has a substantially different value that the approximately $\mathrm{X} / \mathrm{D}=3$ diameters found for the potential core based on the centerline density. This is an interesting result, as in previous works the length of the potential core based on the density has approximately the same value as the one based on the velocity decay, and hypothesizes that the injected, possibly identify by the observation of the scalar fields, may contribute with kinetic energy to the jet.

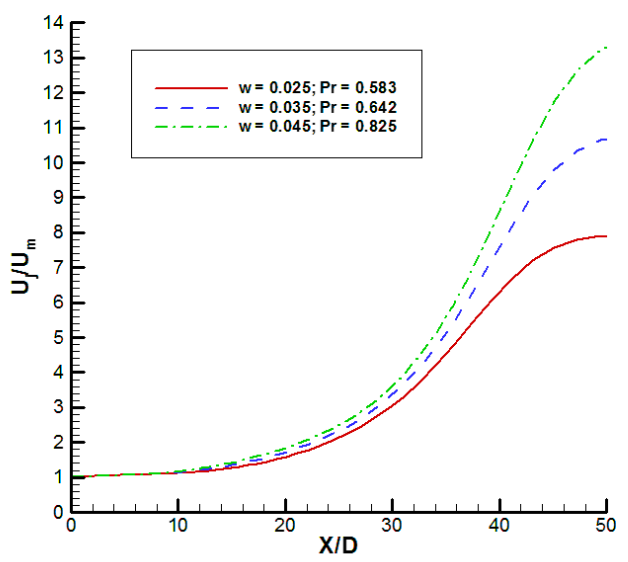

Figure 5 - Centerline velocity decay.

Figure 6 shows the half width of the half maximum of the velocity (HWHMV) for the cases 1,2 and 3 of Table 1 . The three cases have a similar evolution of the HWHMV across the domain of study with an almost linear first stage and a second non linear stage. The tangent of the slope of the almost linear stage corresponds to the jet growing rate. Observing the graphic it is possible to conclude that the jet growing rate is very similar for the three studied cases with only a slightly decrease as the chamber pressure increases. This was however not an expected result as in several previous work the increase of the chamber pressure causes an increase in the jet growing rate.

In Figure 7 are shown the decay rates of velocity and density after the potential core for the cases of Table 1. For both velocity and density an increase in the injection chamber pressure has as result an increase of the decay rate which is in agreement with previous works from other authors, however this effect is more pronounced in the velocity decay. The velocity also shows for the three cases a higher decay rate then the density although is important to remember that the density 
decay starts earlier then the velocity decay due to the longer potential core obtained for the velocity.

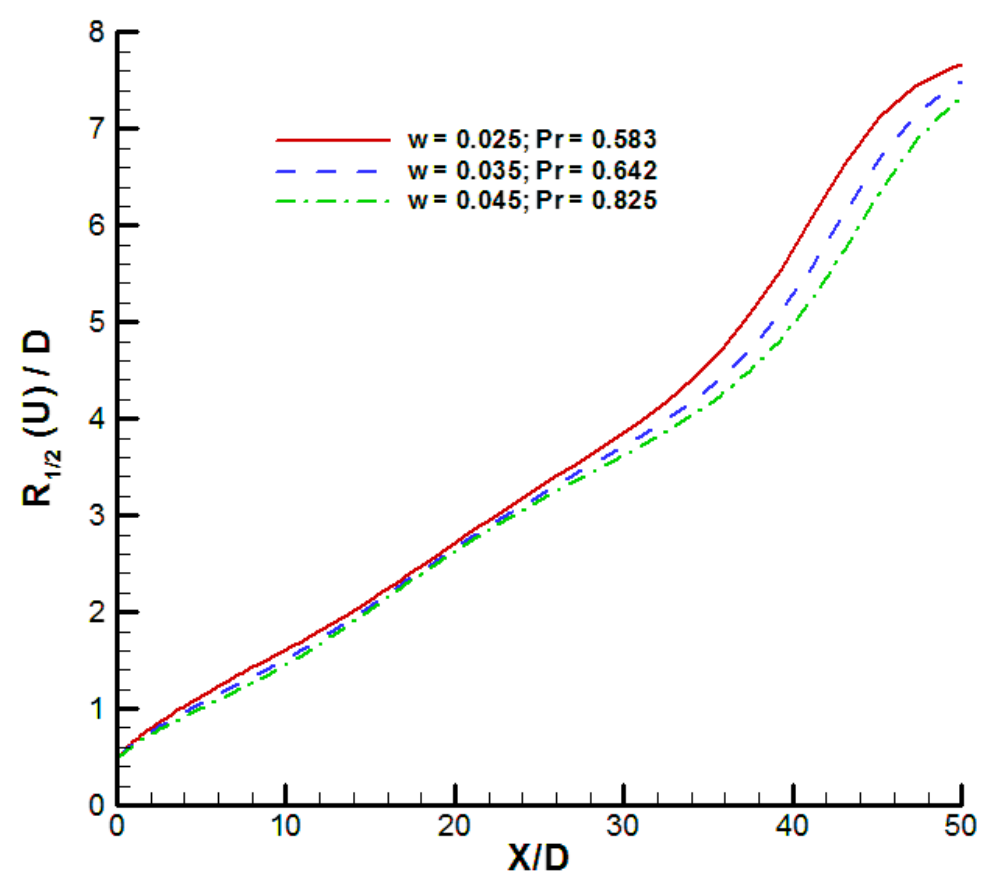

Figure 6-Half width of half maximum of the velocity.

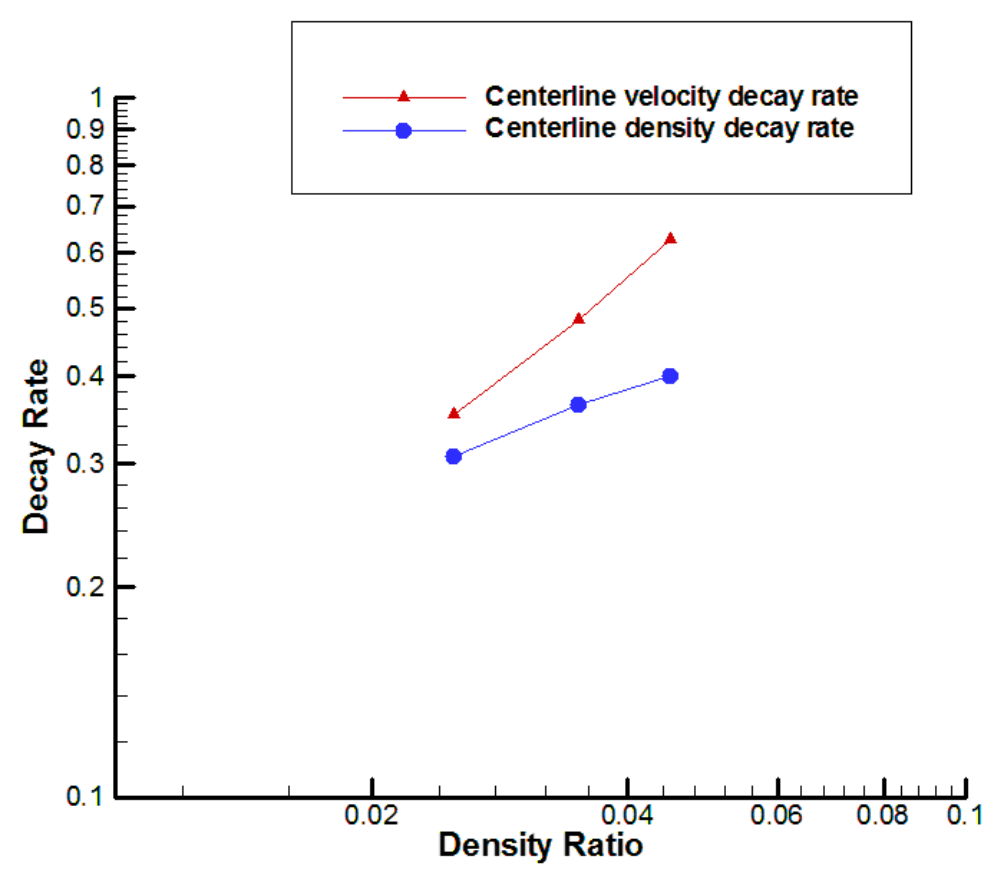

Figure 7 - Decay rate of the centerline velocity and density. 


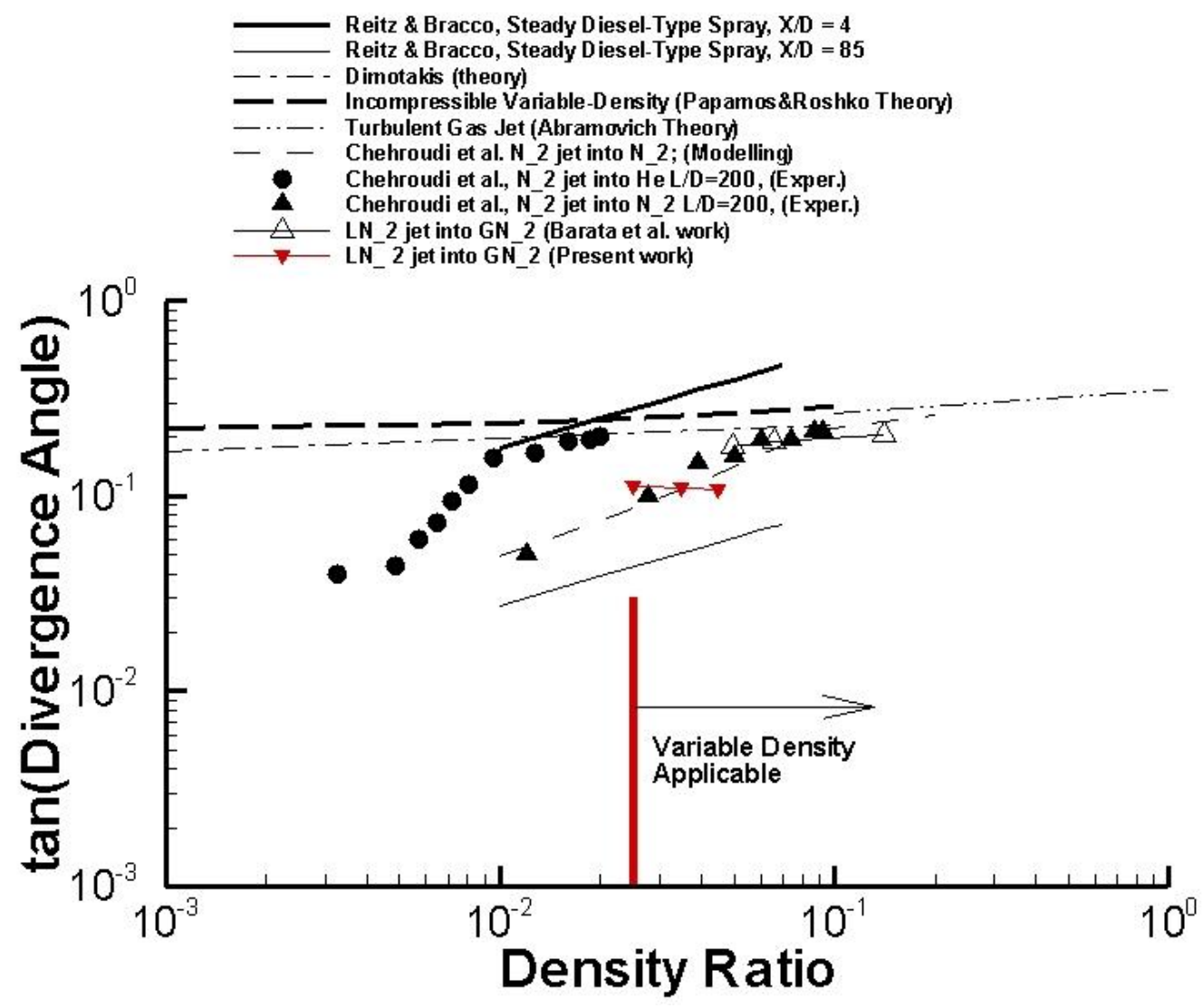

Figure 8 - Tangent of the spreading angle versus the chamber-to-injectant density ratio and representation if limit of applicability of the Variable Density approach.

Figure 8 is similar to the graphic presented by Barata et $a l^{18}$ and shows the tangent of the jet spreading angle, calculated by the half width of half maximum of the velocity, in order to the density ratio. In addition to the three cases of Table 1 the graphic contains also results obtained by other authors in previous investigations including the results of Barata et $a .^{18}$, the investigation which the present work pretends to extend. The results achieved in the present work are represented in red while those achieved by other authors are represented in black. Obversing the graphic it is visible that the result obtained for a density ratio of $\omega=0.025$ agree very well with the experimental result obtained by Chehroudi et $a l^{23}$, the result obtained for a density $\omega=0.035$ is in total agreement with the modeling results of the same author. The $\omega=0.045$ density ratio case shows a slight divergence from previous result but is still very close to the modeling result of Chehroudi et $a l^{23}$. The test of the present variable density approach to lower density ratios, which are not presented here, allow the conclusion that the density ratio of $\omega=0.025$ is the lowest value liable to be modeled by the present mathematical model. This conclusion is represented in Figure 8 by the vertical red line that marks the limit of applicability of the variable density approach.

The influence of the injection velocity in jet behavior was also subject of analysis at the present investigation. The objective was to perform a better characterization of the mathematical model. In order to achieve this objective the variable density approach was tested for different injection velocities as presented in Table 2 .

Figure 9 shows the velocity and scalar fields for the test case 7 of Table 2. It is visible that the jet has the same structure found in Figure 2 corresponding to the case 4 of Table 2. It's however possible to verify that the vortex center appears in localization further downstream, also the mixture fraction and density show a slower evolution across the centerline when compared with the case with slower injection velocity. The axial variation of the centerline density shown in Figure 10 corroborates the observation stated 
a)

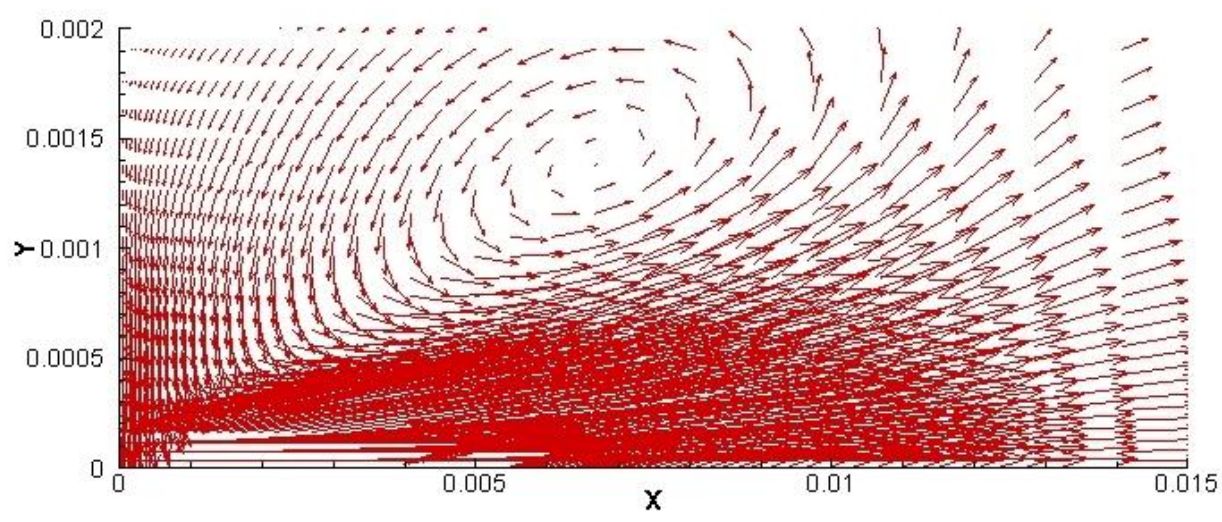

b)

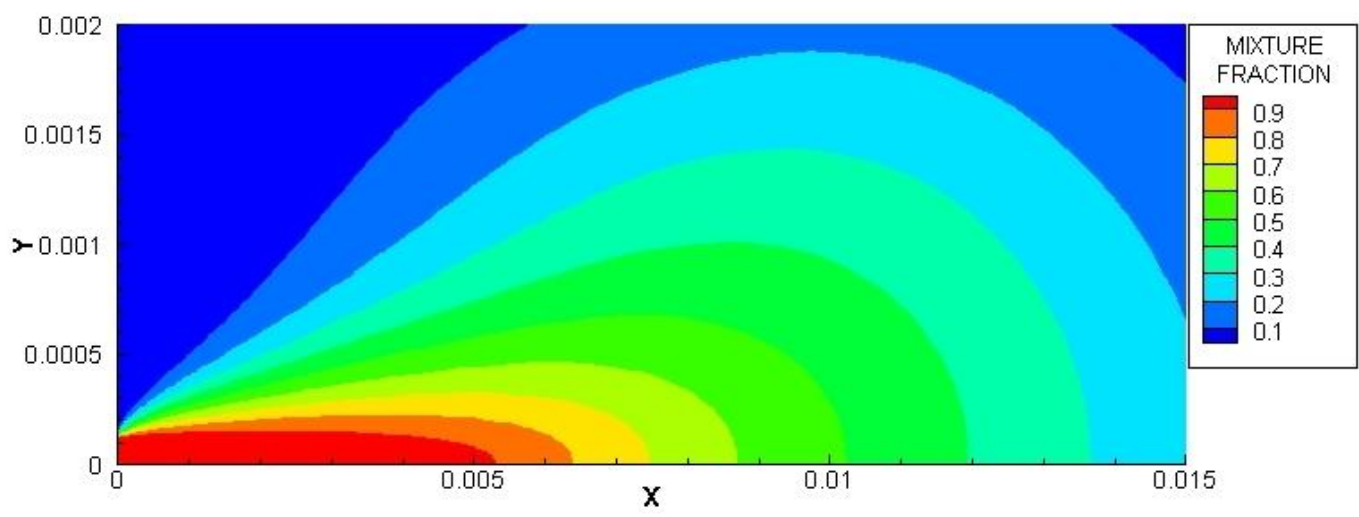

c)

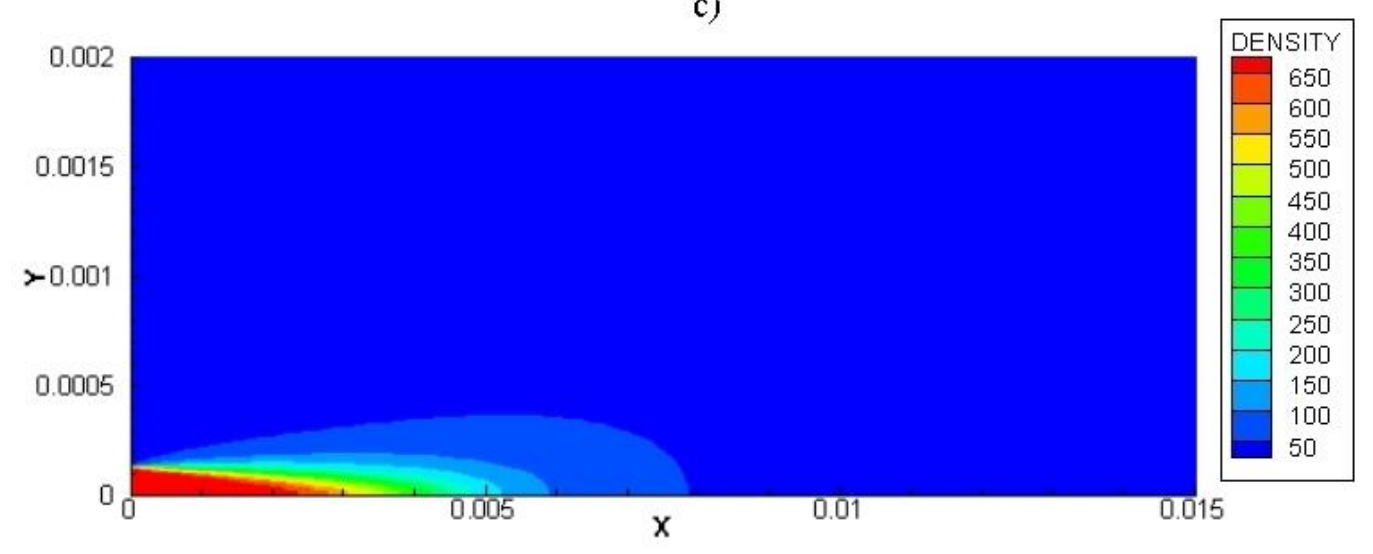

Figure 9 - Velocity and scalar fields of the jet with a density ratio of $\omega=0.025$ and an injection velocity of $U_{\text {in }}$ $=20 \mathrm{~m} \cdot \mathrm{s}^{-1}$, (a) Velocity vectors, (b) Mixture fraction contours, (c) Density contours. 
above. The centerline density shows a slower decrease for higher injection velocities. One interesting observation is the increase of the potential core based on density as the injection velocity increases. Similar observations are made for the centerline velocity decay. As the injection velocity increases the potential core based on the velocity decay increases and the centerline velocity shows a lower rate of decay, this is somehow contrary to the expected since an increase in the injection velocity should lead to an increase of drag forces between the injected and the ambient fluid and this way increase the velocity decay rate. Like in the axial variation of the centerline also for the centerline velocity decay the case 5 with an injection velocity of $U_{\text {in }}=5 \mathrm{~m} . \mathrm{s}^{-1}$ shows a behavior different from the tendency followed by the other three cases.

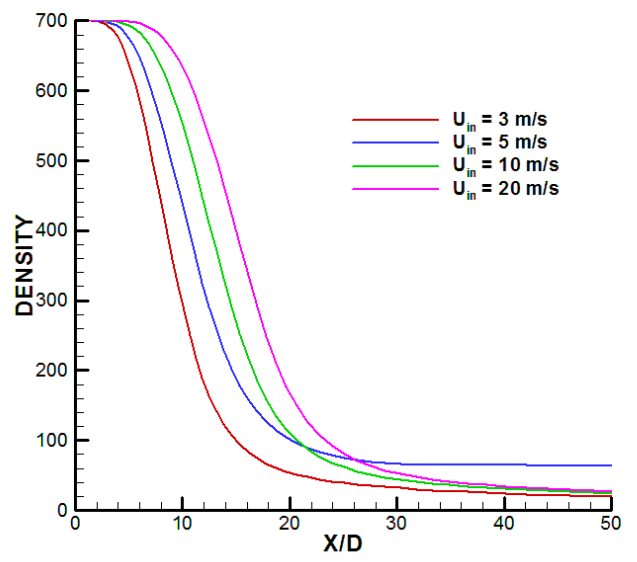

Figure 10 - Axial variation of the centerline density for the cases 4, 5, 6 and 7 of the Table 2 .

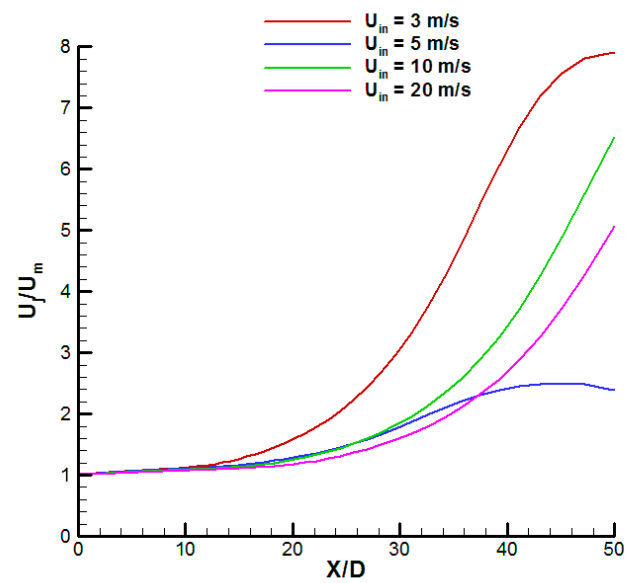

Figure 11 - Centerline velocity decay for the cases 4 , 5,6 and 7 of the Table 2.
The half width of half maximum of the velocity is shown in Figure 11. Again the case 4 of Table 2 shows a different HWHMV structure then the other three cases not having an almost linear stage. By the observation of the graphic can be concluded that an increase of the injection velocity causes a decrease in the jet spreading angle.

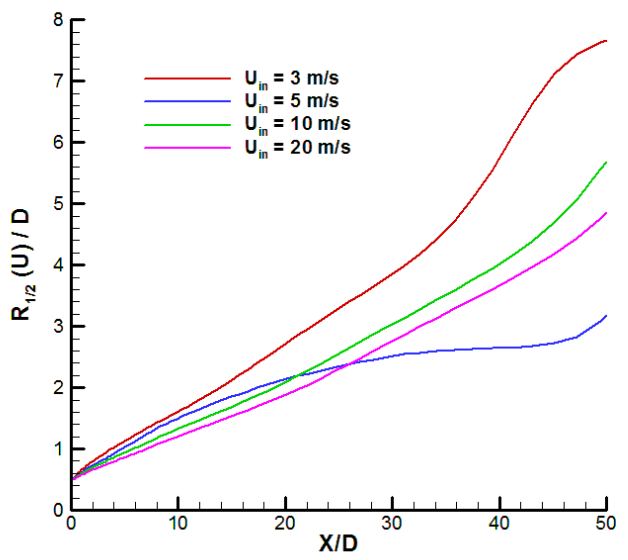

Figure 12 - Half width of half maximum of the velocity for the cases 4, 5, 6 and 7 of the Table 2.

\section{Conclusions}

Barata et al. [18] had concluded that the variable density mathematical model could be successfully used in the study of cryogenic jets injected initially at subcritical temperatures into an environment at a supercritical temperature over a range of subcritical and supercritical pressures [18]. The objective of the present work was to extend the study performed by Barata et al. [18] to lower chamber-to-injection fluid density ratios. Like in Barata et al. [18], for this investigation an injection of cryogenic liquid nitrogen into a gaseous nitrogen environment was simulated.

In the analysis of the obtained result were identified some interesting new results. The fastest mixture fraction scalar field evolution when compared with the density scalar field together with the difference between the potential core based on density and the one based on velocity decay, suggests that a vigorous expansion of the injected fluid happens as it enters the injection chamber. And the longer potential core based on the velocity decay indicated that expansion may somehow contribute with kinetic energy to the jet.

The results of the half with of half maximum of the velocity allowed the determination of the jet growth rate with are in general agreement with the 
results obtained by Chehroudi et al. ${ }^{23}$ described in Ref. 18. By the analysis of the results of the HWHMV obtained to density ratios lower than $\omega$ $=0.025$, it was concluded that below this value no more agreement was found with previous experimental and numerical results. This establishes the limit of applicability of the variable density approach to cryogenic liquid jet into a supercritical temperature and subcritical pressure ambient.

Finally it was concluded that the injection velocity has influence on the results obtained by the variable density approach. A faster injection velocity has as result a delay in mixture process and density decrease. The potential core based both on density and velocity decay increases as the injection velocity increases while the jet growth rate suffers a decrease. This way, the injection velocity must be taken into account when applying the variable density approach to a case study.

\section{$\underline{\text { References }}$}

${ }^{1}$ Chehroudi, B., Cohn, R., and Talley, D., "Visual Characteristics and Initial Growth Rates of Round Cryogenic Jets at Subcritical and Supercritical Pressures," Physics of Fluids, Vol. 14, No. 2, 2002, pp. 850-861.

${ }^{2}$ Bellan, J., "Supercritical (and Subcritical) Fluid Behavior and Modeling: Drops, Streams, Shear and Mixing Layers, Jets and Sprays," Progress in Energy and Combustion Science, Vol. 26, Issues 4-6, 2000, pp. 329-366.

${ }^{3}$ Yang, V., "Modeling of Supercritical Vaporization, Mixing, and Combustion Processes in Liquid-Fueled Propulsion Systems," Proceedings Of the Combustion Institute, Vol. 28, 2000, pp. 925-942.

${ }^{4}$ Segal, C., and Polikhov, S.A., "Subcritical to Supercritical Mixing," Physics of Fluids, Vol. 20, Issue 5, 2008, pp. 052101-1-052101-7.

${ }^{5}$ Oschwald, M., Smith, J. J., Branam, R., Hussong, J., Schik, A., Chehroudi, B. and Talley, D. (2006) "INJECTION OF FLUIDS INTO SUPERCRITICAL ENVIRONMENTS", Combustion Science and Technology, 178: 1, 49100.

${ }^{6}$ Mayer, W.O.H., Schick, A. H. A., Vielle, B., Chauveau, C., Gökalp, I., Talley, D.G., and Woodward, R.D., "Atomization and Breakup of Cryogenic Propellants under High-Pressure Subcritical and Supercritical Conditions," Journal of Propulsion and Power, Vol.14, No.5, 1998, pp.835-842.

${ }^{7}$ Chehroudi, B., Cohn, R., and Talley, D., "Cryogenic Shear Layers: Experiments and Phenomenological Modeling of the Initial Growth Rate under Subcritical and Supercritical
Conditions," International Journal of Heat and Fluid Flow, Vol. 23, Issue 5, 2002, pp. 554-563.

${ }^{8}$ Givler, S.D., John Abraham, J., "Supercritical Droplet Vaporization and Combustion Studies," Progress in Energy and Combustion Science, Vol. 22, Issue 1, 1996, pp. 1-28.

${ }^{9}$ Zhang, H., Raghavan, V., Gogos, G., "Subcritical and Supercritical Droplet Evaporation within a Zero-Gravity Environment: Low Weber Number Relative Motion," International Communications in Heat and Mass Transfer, Vol. 35, Issue 4, 2008, pp. 385-394.

${ }^{10}$ Nomura, H., Ujiie, Y., Rath, H.J., Sato, J., Kono, M., "Experimental Study of High-Pressure Droplet Evaporation using Microgravity Conditions," Twenty-Sixth Symposium (International) on Combustion/The Combustion Institute, 1996, pp. 1267-1273.

${ }^{11}$ Tsue, M., Nomur, H., Niwa, M., Miyano, H., Sato, J., Kono, M., "Evaporation of a Fuel Droplet in a High Pressure High Temperature Atmosphere," Japan Society for Aeronautical and Space Sciences, Journal (ISSN 0021-4663), Vol. 37, No. 420, 1989, pp. 21-28.

${ }^{12}$ Fieberg, C., Reichelt, L., Martin, D., Renz, U., and Kneer, R., "Experimental and Numerical Investigation of Droplet Evaporation under Diesel Engines Conditions," International Journal of Heat and Mass Transfer, Vol. 52, Issues 15-16, 2009, pp. 3738-3746.

${ }^{13}$ Zhu, G.S., and Aggarwal, S.K., "Transient Supercritical Droplet Evaporation with emphasis on the Effects of Equation of State", International Journal of Heat and Mass Transfer, Vol. 43, Issue 7, 2000, pp. 1157-1171.

${ }^{14}$ Emekwuru, N.G., Watkins, P., "Analysis of a Two-Fluid Sprayer and its Use to Develop the Number Size Distribution Moments Spray Model Part II: Computational Analysis," Atomization and Sprays, Vol. 20, Issue 8, 2010, pp. 653-672.

${ }^{15}$ Oschwald, M., and Schik, A., "Supercritical Nitrogen Free Jet Investigated by Spontaneous Raman Scattering," Experiments in Fluids, Vol. 27, No. 6, 1999, pp. 497-506.

${ }^{16}$ Mayer, W., and Telaar, J., "Investigation of Breakup of Turbulent Cryogenic Variable-Density Jets," Atomization and Sprays, Vol. 12, Issue 5-6, 2002, pp. 651-666.

${ }^{17}$ Sanders, J. P. H., B. Sarh, B., Gokalp, I., "Variable Density Effects in Axisymmetric Isothermal Turbulent jets: a Comparison Between a First- and a Second-order Turbulence Model," Int. J. Heat Mass Transfer. Vol. 40, No. 4, 1997, pp. 823-842.

${ }^{18}$ Barata, J.M.M., Gokalp, I., Silva, A.R.R., "Numerical Study of Cryogenic Jets under Supercritical Conditions," Journal of Propulsion and Power, Vol. 19, No.1, 2003, pp. 142-147.

${ }^{19}$ Zong, N., Meng, H., Hsieh, S., and Yang, V., "A Numerical Study of Cryogenic Fluid Injection 
and Mixing under Supercritical Conditions," Physics of Fluids, Vol. 16, No. 12, 2004, pp. 42484261

${ }^{20}$ Sierra-Pallares, J., Parra-Santos, M.T., García-Serna, J., Castro, F., Cocero, M.J., "Numerical Analysis of High-pressure Fluid Jets: Application to RTD Prediction in Supercritical Reactors", Journal of Supercritical Fluids, Vol. 49, 2009, pp. 249-255.

${ }^{21}$ Schmitt, T., Selle, L., Cuenot, B., and Poinsot, T., "Large-Eddy Simulation of Transcritical Flows", Comptes Rendus Mécanique, Vol. 337, Issues 6-7, June-July 2009, pp. 528-538.

${ }^{22} \mathrm{Kim}, \mathrm{T} .$, Kim, Y., Kim, S., "Numerical study of cryogenic liquid nitrogen jets at supercritical pressures," The Journal of Supercritical Fluids, Vol. 56, Issue 2, 2011, pp. 152-163.

${ }^{23}$ Chehroudi, B., Cohn, R., and Talley, D., "Spray/Gas Behaviour of Cryogenic Fluids under Sub- and Supercritical Conditions", Eighth International Conference on Liquid Atomization \& Sprays Systems, ICLASS-2000, Pasadena, California, USA, July 16-20, 2000. 